US Army Corps

of Engineers

Waterways Experiment

Station

Natural Resources Technical Support Program

\title{
Estimating the Local Economic Impacts of Recreation at Corps of Engineers Projects - 1996
}

by Dennis B. Propst, Daniel J. Stynes, Wen-Huei Chang, Michigan State University

R. Scott Jackson, WES 
The contents of this report are not to be used for advertising, publication, or promotional purposes. Citation of trade names does not constitute an official endorsement or approval of the use of such commercial products.

The findings of this report are not to be construed as an official Department of the Army position, unless so designated by other authorized documents. 


\section{Estimating the Local Economic Impacts of Recreation at Corps of Engineers Projects - 1996}

by Dennis B. Propst, Daniel J. Stynes, Wen-Huei Chang

Department of Park and Recreation Resources

Michigan State University

East Lansing, MI 48824

R. Scott Jackson

U.S. Army Corps of Engineers

Waterways Experiment Station

3909 Halls Ferry Road

Vicksburg, MS 39180-6199

Final report

Approved for public release; distribution is unlimited 


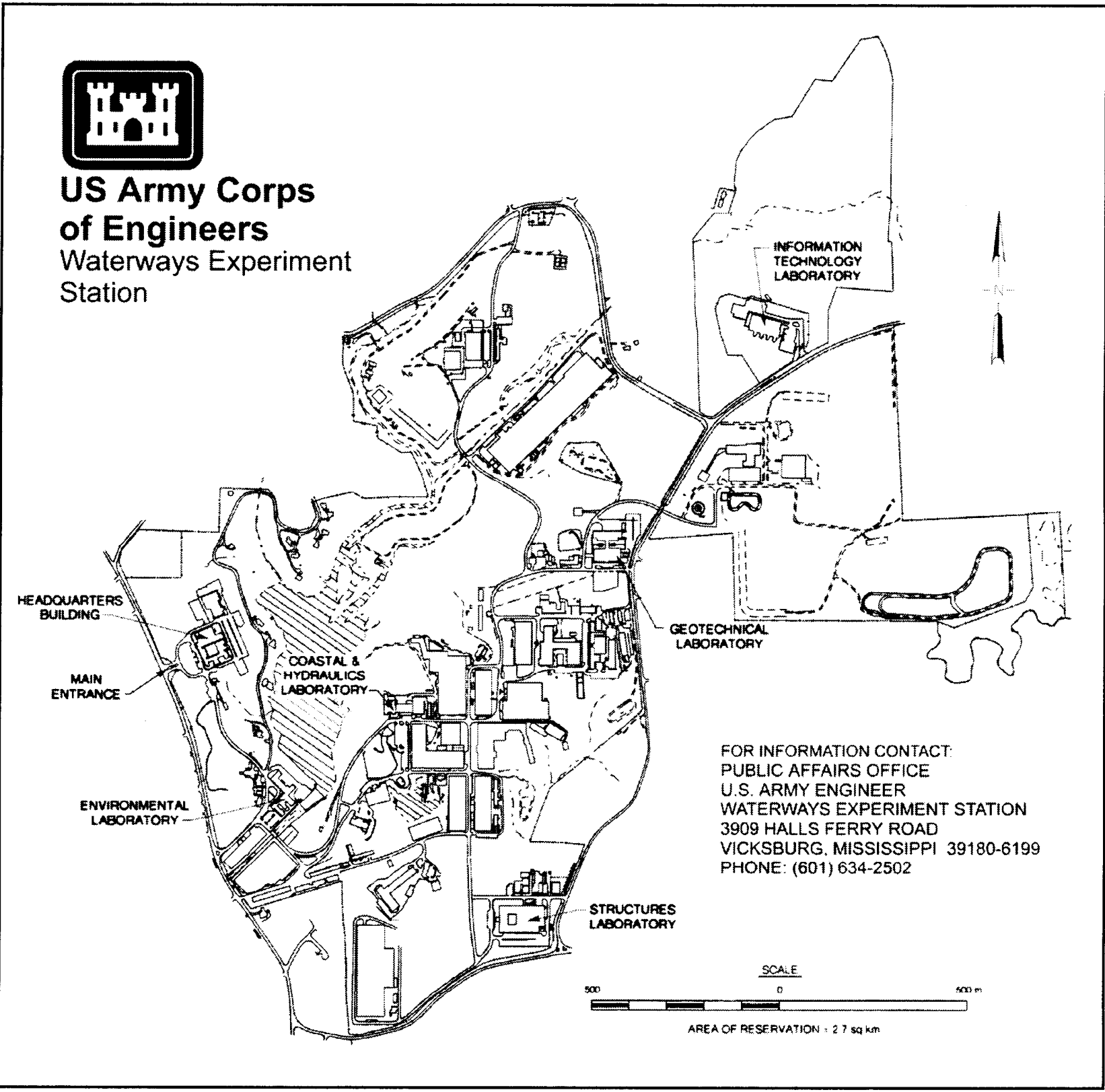

\section{Waterways Experiment Station Cataloging-in-Publication Data}

Estimating the local economic impacts of recreation at Corps of Engineers projects-1996 / by Dennis B.

Propst ... [et al.] ; prepared for U.S. Army Corps of Engineers.

63 p. : ill. ; $28 \mathrm{~cm}$. - (Technical report ; R-98-1)

Includes bibliographic references.

1. Recreation areas - United States - Economic aspects. 2. Recreation areas - Public use United States. 3. Economic forecasting - United States. 4. Public works - United States - Economic aspects. I. Propst, Dennis B. II. United States. Army. Corps of Engineers. III. U.S. Army Engineer Waterways Experiment Station. IV. Natural Resources Technical Support Program (U.S.) V. Series: Technical report (U.S. Army Engineer Waterways Experiment Station) ; R-98-1.

TA7 W34 no.R-98-1 


\section{Contents}

Preface ..................... v

1 -Introduction . ...................... 1

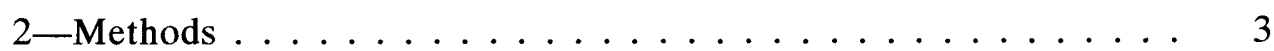

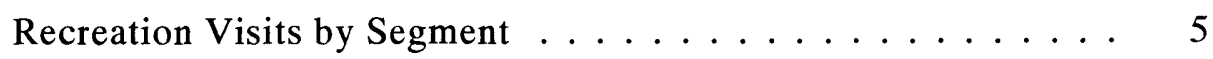

Visitor Spending Averages . . . . . . . . . . . . 7

Multipliers ................... 8

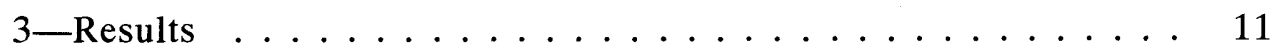

Project Level Results . . . . . . . . . . . . . . . . . 17

Assumptions and Sources of Error f . . . . . . . . . . 18

District and Division Impacts . . . . . . . . . . . . 19

National Economic Effects . . . . . . . . . . . . . 20

4-Errors in Applying the Simplified Approach . . . . . . . . . . 21

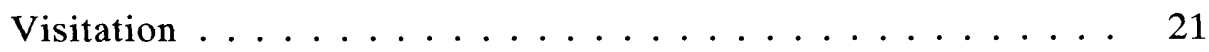

Spending Averages $\ldots \ldots \ldots \ldots \ldots \ldots \ldots$

Multipliers ........................ 23

Cumulative Effect of Errors . . . . . . . . . . 24

5 -Conclusions and Recommendations ............. 25

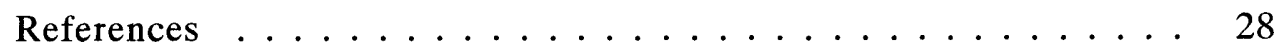

Appendix A: Worksheet for Estimating the Economic Impacts of Visitor Spending at Corps of Engineers Projects . . . . . . . . A1

Appendix B: Summary Results for all Corps of Engineers Projects . . B1

Appendix C: Economic Multipliers for Regions Surrounding 108 Corps of Engineers Projects

SF 298 


\section{List of Tables}

Table 1. Summary of Recreation Visits to CE Projects, 1996 . . 7

Table 2. CE Visitor Spending Profiles by Segment . . . . . . 8

Table 3. Economic Multipliers for Regions Surrounding 108 CE Projects . . . . . . . . . . . . . 9

Table 4. Visits by Segments for 36 Selected CE Projects . . . . . 12

Table 5. Regional Economic Impacts for 36 Selected CE Projects: Sales ........................ 13

Table 6. Regional Economic Impacts for 36 Selected CE Projects: Income ................... 14

Table 7. Regional Economic Impacts for 36 Selected CE Projects: Jobs ..................... 15

Table 8. Summary Results for All CE Districts . . . . . . . . 16

Table 9. Summary of Total CE Visits and Spending . . . . . . . 17

Table 10. Economic Impacts of Recreation Visitor Spending on 456 CE Projects . . . . . . . . . . . 17 


\section{Preface}

The work reported herein was conducted as a part of the Natural Resources Technical Support (NRTS) program. The NRTS is sponsored by the Headquarters, U.S. Army Corps of Engineers (HQUSACE), and is assigned to the U.S. Army Engineer Waterways Experiment Station (WES) under the purview of the Environmental Laboratory (EL). Funding was provided under the Department of Army Appropriation No. 96X3123, Operation and Maintenance. The NRTS was managed by Dr. David J. Tazik.

This report was prepared by Dr. Dennis B. Propst, Dr. Daniel J. Stynes, and Mr. Wen-Huei Chang, Department of Recreation Resources, Michigan State University, and Mr. R. Scott Jackson, EL.

The study was supervised by Dr. H. Roger Hamilton, Chief, Resource Analysis Branch, Natural Resources Division (NRD), EL. General supervision was provided by Dr. Tazik, Chief, NRD, and Dr. John Harrison, Director, EL. Technical Reviewers were Ms. Kathleen Perales and Mr. Jim Henderson, EL.

At the time of publication of this report, Director of WES was Dr. Robert W. Whalin. Commander was COL Robin R. Cababa, EN.

This report should be cited as follows:

Propst, D. B., Stynes, D. J., Chang, W-H., and Jackson, R. S. (1998).

"Estimating the local economic impacts of recreation at Corps of Engineers projects - 1996," Technical Report R-98-1, U.S. Army Engineer Waterways Experiment Station, Vicksburg, MS.

The contents of this report are not to be used for advertising, publication, or promotional purposes. Citation of trade names does not constitute an official endorsement or approval of the use of such commercial products. 


\section{Introduction}

The U.S. Army Corps of Engineers (CE) manages 456 water resource development projects throughout the United States. These lake and river projects provide significant recreation opportunities and benefits to visitors. Spending by visitors on goods and services associated with CE recreation opportunities generates sales, income, and jobs in local regions surrounding CE projects. The magnitude of these economic effects varies from project to project due to complex interactions between project characteristics (e.g., extent and type of recreation facilities) and regional economic characteristics (e.g., population size and number and type of retail establishments available for visitor spending). In this report, a simplified approach for estimating the economic impacts of recreation at the individual project level is presented. The approach focuses on aggregate estimates of impacts that can be derived using data available from the sources described in this report.

The purpose of this report is to estimate the economic effects of recreationists' spending within the local region surrounding each of the $456 \mathrm{CE}$ projects. Such information is needed because decisions that affect project operations are based partially on the importance of the project to regional and national economies. Realistic estimates used to make such decisions stem from processes like the one presented in this report. In previous reports, impacts for 12 individual projects (Propst, Stynes, and Rutz 1995a-f; Stynes, Propst, and Rutz 1995g-1) and the Upper Mississippi River System (Carlson et al. 1995) have been estimated. Impacts of CE recreation activity have also been estimated at the national (Jackson, Stynes, and Propst 1994; Jackson et al. 1996) and State levels (Jackson et al. 1996).

At the national level in 1991 , over $\$ 6$ billion was spent by CE recreation visitors for trip-related items, resulting in $\$ 8$ billion in household income and 404,000 jobs in industries directly and indirectly supplying goods and services to CE visitors (Jackson, Stynes, and Propst 1994). In 1994 , these national estimates rose to $\$ 7.7$ billion in trip-related spending, $\$ 14.7$ billion in household income, and 443,000 jobs (Jackson et al. 1996). On a State-by-State basis, visitor spending ranged from $\$ 744$ million on trips to CE projects in Arkansas to about $\$ 2$ million on trips to CE projects in Alaska and Arizona. The employment effects of visitor spending in 
these States ranged from 25,000 total jobs in Arkansas to around 40 jobs in Alaska and Arizona (Jackson et al. 1996). In the 76-county region along the Upper Mississippi River, $\$ 192$ million in total trip and durable goods spending was associated with $\$ 90$ million in employee wages and 5,800 jobs (Carlson et al. 1995).

Results cited above have all made use of the MI-REC system (Stynes and Propst 1996) to estimate visitor spending and economic impacts. MI-REC produces detailed estimates of spending for up to 12 visitor segments within as many as 33 spending categories. The MI-REC procedures bridge spending to an input-output model for the region surrounding a given project and estimate direct, indirect, induced, and total effects for as many as 528 economic sectors. MI-REC procedures require that users have access to the IMPLAN input-output modeling system (Alward and Palmer 1983; Taylor et al. 1993) and that they be sufficiently familiar with regional economic concepts and spreadsheet and IMPLAN software to carry out the analysis. Individual CE projects generally do not have the time and resources to carry out this analysis. Therefore, this report provides a modification of these procedures that can be carried out at the project level using available data and a simple worksheet/spreadsheet. The simplified procedures also permit the estimation of local economic impacts for all $456 \mathrm{CE}$ projects.

The MI-REC procedures are simplified in several ways. Most importantly, the need to estimate an input-output model for the local region has been eliminated. Instead, guidance in selecting appropriate aggregate multipliers for a particular area is provided. The simplified procedures yield only aggregate estimates of spending, sales, income, and jobs. Spending patterns are generalized from previous studies, and visitation and segment shares are derived from the Corps' Natural Resource Management System (NRMS) database. The resulting simplifications provide a worksheet similar to the National Park Service's Money Generation Model (U.S. Department of Interior 1990). The worksheet is tailored to make use of the CE visitation and revenue databases, and the default parameters used in the model are derived from surveys and regional economic models for projects chosen to be representative of CE projects.

In order to develop this simplified approach, some judgment and assumptions were required to generalize visitor spending profiles and multipliers to projects where original data were not collected. Local project managers should play an active role in evaluating and modifying the assumptions and the values upon which these estimates were built. The simplifications and the worksheet were designed to facilitate the evaluation process at the project level. 


\section{Methods}

Four factors must be estimated and multiplied together (Equation 1) to determine the economic impacts of visitor spending on a region: number of visits, spending per visitor, capture rate, and multipliers.

$$
\text { Economic impact }=\text { \# of visits } \times \text { average spending per visit } \times
$$

capture rate $\times$ regional economic multiplier

Visitation estimates come from counts of visitors reported annually by the CE in the NRMS database. These must be converted to party days (the units in which spending is measured) and then divided among the segments for which spending profiles have been estimated. Spending profiles come from visitor surveys at individual CE projects or can be adapted from studies at similar sites, as has been done in this report. Total spending is obtained by multiplying the per trip spending average of each segment by the number of trips by that segment and then summing across segments. Total spending is multiplied by a capture rate, which is the proportion of total visitor spending that is "captured" by a region's economy (i.e., the part that does not escape because of leakages to sectors outside the region). When total spending is applied to regional economic models for local regions around CE projects, the model indicates how much of that spending is captured. Regional economic impacts are then estimated by multiplying the "captured" total spending by regional economic multipliers. Multipliers are derived from regional economic models for the local regions around $\mathrm{CE}$ projects or can be taken from published multipliers for a region of a similar size and economic structure.

Three variations of Equation 1 are used to estimate the economic impacts of recreation. The $456 \mathrm{CE}$ projects are divided into three groups, and a slightly different estimation approach is used for each group. The first group consists of the 12 projects included in the 1989-90 visitor spending study (Propst et al. 1992). Spending profiles were estimated from visitors at these projects, and local input-output models were estimated to derive multipliers for these regions. For these 12 projects, all three of the factors in Equation 1 are estimated with data specific to the particular projects, although spending profiles still must be generalized 
over time using price indices. Due to small sample sizes within segments for individual projects, the spending averages are estimated using cases from all 12 projects.

In the second group are 96 projects for which local input-output models were estimated to derive the multipliers (Becker 1997). Using IMPLAN, the models were estimated for counties within $48 \mathrm{~km}$ (30 miles) of project boundaries. ${ }^{1}$ Spending profiles were generalized from the segment averages for visitors to the 12 lakes in the first group. No spending surveys were conducted at these 96 projects, so it was assumed that, at least within segments, the averages for the 12 lakes in Group One may be applied.

For the final 348 projects in Group Three, both the spending averages and the multipliers were generalized from the previous two groups. Spending averages by segment are assumed to be the same as for the 12 lakes surveyed in 1989-90. Multipliers are derived by averaging the multipliers obtained from the input-output models constructed for the 108 projects in Groups One and Two. These 348 projects illustrate how both spending and multipliers may be generalized from other sites.

The variations of Equation 1 illustrate different assumptions made in order to generalize spending profiles and regional economic multipliers to Groups Two and Three projects. The observed variation in spending and multipliers across projects is reported to provide an indication of how much error may be involved in generalizing the averages to a particular project. More reliable estimates can be made for individual projects by conducting new visitor spending surveys at each project and estimating a regional economic model for the counties surrounding the project. However, this approach entails considerable cost for the surveys and requires access to and familiarity with regional economic models. Such primary data collection and modeling will be possible only in selected cases. Estimates based on secondary data and simple models rest heavily on the generalizability of the spending averages and multipliers from one project or region to another. The visitor segmentation approach implicit in the MI-REC system helps to capture variations in spending across different projects that are explained by the mix of visitors attracted. Differences in total spending are explained considerably by the differences in the number and types of visitors.

The simpler approaches illustrated in this report yield quick estimates of spending and impacts for all $456 \mathrm{CE}$ projects. The estimates for individual projects may be refined by adjusting the spending averages or multipliers based on a good understanding of the visitors to a particular project and the economy of the surrounding region. Included in this report is a simple worksheet for computing economic impacts based on project specific-visitation data, existing spending profiles, and multipliers for

1 The decision rules for selecting the counties that define local regions around the $96 \mathrm{CE}$ projects are found in Becker (1997). 
$108 \mathrm{CE}$ projects. CE project managers can easily update and refine estimates of spending and economic impacts for their project by entering new visitation figures and using some judgement to adjust or choose spending averages and multipliers that reflect their project and region.

A more detailed discussion of the procedures used to estimate visits, spending, and multipliers is provided in the remainder of this section. The steps for estimating economic impacts for a particular project are also illustrated on the worksheet in Appendix A, using Mark Twain Lake in Missouri as an example.

\section{Recreation Visits by Segment}

Recreation visitation was estimated using the PR_USE and CUR_FEE databases from the NRMS system. PR_USE contains a project-level reporting of total visitation and the percentages of campers, day users, and boaters among all visitors. These percentages are based on surveys and are therefore subject to sampling and other survey errors. Not all projects have conducted surveys in recent years; therefore, the estimates may be dated if use patterns have changed over time. CUR_FEE contains a siteby-site reporting of all the revenues and fees collected each year.

CUR_FEE was thought to be a more accurate measure of camping visitation than PR_USE because CUR_FEE is updated annually. PR_USE is based on surveys that may have been conducted a number of years in the past. Total CE camping visitation nationally was 29.8 million visits in 1996 based on the PR_USE database, whereas camping visitation derived from the CUR_FEE database was only 7.6 million visits. The former is almost four times the revenue-based estimate.

The revenue-based estimate was assumed to be more accurate than the PR_USE data, although several assumptions were required to convert camping revenues to camper visits. Total campsite revenues (CG_FEE_REV) were divided by an average fee per night to estimate the number of camper party nights. An average fee of $\$ 8$ per campsite per night was used taking into account fees at sites with and without electricity, fees at CE-managed and non-CE-managed facilities, and discounts for holders of Golden Age and Golden Access Passports. Party nights at CE-managed sites were then expanded to include non-CE-managed sites by assuming similar occupancy rates at $\mathrm{CE}$ - and non-CE-managed sites.

The number of camping party days was then converted to camper "visits" using an average camper length of a stay of 2.8 days and party size of 3.4. Camping party days must be converted to person visits to be consistent with the units for which total and day-use visitation are reported in the NRMS system (PR_USE database). A "visit" is defined as the entry of one person onto a CE project to engage in one or more recreation activities. As used in this study, a "person trip" is equivalent to a visit. 
The number of day-use visits was derived by subtracting camping visits from total visits. Visitors who stay in hotels and other off-project lodging accommodations but visit the project for part of a day are treated as day users by the CE. Since these "other overnight" visitors have significantly different spending patterns than typical day users (Propst et al. 1992), it is important to separate them from day users to estimate spending. No information was available to estimate the percentage of day users staying overnight in the area. Following the assumption made in the 1994 study (Jackson et al. 1996), 1 percent of day users was set aside as other overnight visitors. This percentage should be adjusted for individual projects based on local information.

Campers, day users, and other overnight visitors were further divided into boaters and nonboaters based upon the proportion of boaters reported by each project in the 1996 NRMS database system. This resulted in six visitor segments:

a. Campers who boat.

b. Campers who do not boat.

c. Day users who boat.

d. Day users who do not boat.

$e$. Other overnight visitors who boat.

f. Other overnight visitors who do not boat.

Estimates of the number of visitors for each segment in person trips (visits) were converted to party days using average lengths of stay and party sizes for each segment (Table 1 ). The conversion to party days improves the ability to generalize spending profiles across projects. Visitor spending was originally measured in party trips. However, the use of party trips requires the questionable assumption that visitor length of stay does not vary across projects. Another reason for the conversion is that the party day is more similar than the party trip to the units in which the CE measures visitation. Information on party size is all that is needed to convert visits to party days, and party sizes tend to be more consistent across projects than lengths of stay. The formula for converting visits to party days is as follows:

$$
\text { party days }=\text { person visits } \times \frac{\text { average length of stay }}{\text { average party size }}
$$

Length-of-stay and party-size parameters were estimated based on Propst et al. (1992) and represent national averages. They may be adjusted on the worksheet (Appendix A) to better fit individual projects. Table 1 
illustrates the conversion from person visits to party days at the national level. There were 375.7 million recreation visits to the $456 \mathrm{CE}$ projects in 1996. This figure converts to 139.4 million party days. Summing across boaters and nonboaters in Table 1, day users accounted for 92.7 percent of all party days; campers, 4.5 percent; and other overnight visitors, 2.8 percent. Twenty-two percent of all party days were by boaters, 78 percent by nonboaters. The proportions of visitors from each segment vary widely for individual projects (see Appendix B).

\section{Table 1}

Summary of Recreation Visits to CE Projects, 1996

\begin{tabular}{|c|c|c|c|c|c|c|c|}
\hline & \multicolumn{2}{|c|}{ Camper ${ }^{1}$} & \multicolumn{2}{|c|}{ Day User ${ }^{2}$} & \multicolumn{2}{|c|}{ Other Overnight ${ }^{3}$} & \multirow[b]{2}{*}{ Total } \\
\hline & Boat & Nonboat & Boat & Nonboat & Boat & Nonboat & \\
\hline Visits (person trips, MM) & 1.7 & 6.0 & 80.4 & 283.9 & 0.8 & 2.9 & 375.7 \\
\hline $\begin{array}{l}\begin{array}{l}\text { Average length of stay } \\
\text { (days) }\end{array} \\
\end{array}$ & 3.0 & 2.7 & - & - & 3.8 & 3.1 & - \\
\hline Average party size $^{4}$ & 3.6 & 3.3 & 2.9 & 2.8 & 3.4 & 2.9 & - \\
\hline Visits (party days, MM) & 1.4 & 4.9 & 27.7 & 101.4 & 0.9 & 3.0 & 139.4 \\
\hline
\end{tabular}

${ }^{1}$ Number of campers in party days was derived from the 1996 NRMS, CUR_FEE database by dividing total camping revenue by an average of $\$ 8.00$ per party day camping fee and expanding by the number of non-CE-managed campsites (see Appendix A for example). Then, number of campers in person trips = number of party days $\times$ average party size $\div$ average length of stay. Percent of boaters was obtained from the NRMS, PR_USE database.

${ }^{2}$ Number of day users in person trips was derived from the 1996 NRMS, PR USE database by subtracting camper visits from total visits (see Appendix A for example). Then, number day users in party days = number of person trips $\times$ average length of stay $\div$ average party size. Percent of boaters was obtained from NRMS, PR_USE database.

${ }^{3}$ Assume that 1 percent of day users stayed overnight in lodging accommodations outside of project boundaries.

${ }^{4}$ From 1994 national and State economic impact study (Jackson et al. 1996). The proportions of boaters and nonboaters were estimated from the results of the 12-lake study (Propst et al. 1992).

\section{Visitor Spending Averages}

Spending profiles for each of the six segments were estimated from the 1989-90 12-lake survey and price adjusted to 1996 dollars (Table 2). A spending profile shows the average amount spent per party per day for a given visitor segment. Trip spending includes spending on goods and services consumed during a trip such as gasoline, food, and lodging. Expenditures for durable goods (items like boats and recreational vehicles (RVs) that are used on multiple trips) were not included. Total visitor spending is obtained by multiplying average per party day spending by the number of party days for each segment and then summing the results across segments.

Typical CE visitors (i.e., the weighted average of the six segment spending profiles) in 1996 spent $\$ 40.15$ per party per day within $48 \mathrm{~km}$ (30 miles) of the project (Table 2). Average spending varied from $\$ 129.36$ per party per day for other overnight visitors who boat, to $\$ 33.24$ per party per day for day users who do not boat. These national averages may be replaced in the worksheet (Appendix A) by project-level visitor expenditure data, when such data are available and accurate. 


\section{Table 2 \\ CE Visitor Spending Profiles by Segment (per party per day, in 1996 dollars) ${ }^{\uparrow}$}

\begin{tabular}{|c|c|c|c|c|c|c|c|}
\hline \multirow[b]{2}{*}{ Spending Category } & \multicolumn{2}{|c|}{ Camper } & \multicolumn{2}{|c|}{ Day User } & \multicolumn{2}{|c|}{ Other Overnight } & \multirow[b]{2}{*}{ Average $^{2}$} \\
\hline & Boat & Nonboat & Boat & Nonboat & Boat & Nonboat & \\
\hline Hotels and motels & 3.32 & 1.46 & 0.00 & 0.00 & 56.43 & 42.00 & 1.37 \\
\hline Camping & 10.64 & 8.27 & 0.00 & 0.00 & 1.83 & 2.09 & 0.46 \\
\hline Grocery & 14.76 & 13.21 & 11.45 & 7.64 & 15.33 & 9.00 & 8.75 \\
\hline Restaurant & 5.52 & 5.11 & 3.81 & 8.14 & 13.89 & 13.10 & 7.29 \\
\hline Automobile and $\mathrm{RV}^{3}$ & 7.92 & 12.28 & 9.54 & 6.24 & 7.84 & 8.52 & 7.18 \\
\hline Boating $^{4}$ & 14.22 & 0.00 & 18.01 & 0.00 & 20.84 & 0.00 & 3.89 \\
\hline Fishing and hunting ${ }^{5}$ & 1.78 & 0.73 & 1.94 & 0.81 & 2.67 & 3.26 & 1.11 \\
\hline $\begin{array}{l}\text { Recreation and } \\
\text { entertainment }^{6}\end{array}$ & 1.07 & 2.65 & 1.77 & 1.48 & 3.07 & 3.49 & 1.63 \\
\hline Miscellaneous ${ }^{7}$ & 6.69 & 5.81 & 7.73 & 8.94 & 7.47 & 5.72 & 8.49 \\
\hline Total & 65.92 & 49.52 & 54.25 & 33.25 & 129.37 & 87.18 & 40.17 \\
\hline \multicolumn{8}{|c|}{$\begin{array}{l}1 \text { Visitor trip-expenditure averages within } 48 \mathrm{~km} \text { ( } 30 \text { miles) of CE projects from Propst et al. (1992). } \\
2 \text { Averages are weighted by the number of visits by each of the six segments. } \\
{ }^{3} \text { Includes gas, oil, rental, repairs, washing, and parts. } \\
{ }^{4} \text { Includes gas, oil, rental, repairs, parts, launch and transient slip fees, and boat fares on rivers, lakes, or canals. } \\
5 \text { Includes temporary license fees (excludes annual license), fishing-boat charter fees, fishing bait, and ammunition } \\
\text { and handloading materials for hunting. } \\
{ }^{6} \text { Includes rental fees for recreation equipment, fees for guide services, admission to spectator sports or tourism } \\
\text { attractions, and other recreation expenses (bowling, golfing, etc.) } \\
{ }^{7} \text { Includes film purchasing and developing, video tape purchasing and processing, souvenirs and gifts, clothing, } \\
\text { and all other expenses not listed above. }\end{array}$} \\
\hline
\end{tabular}

\section{Multipliers}

Multipliers for regions around CE projects were estimated using regional models constructed with IMPLAN. For each of the 108 projects in Groups One and Two, counties within a $48-\mathrm{km}(30-$ mile) radius of the project were combined to form the local region (see Becker (1997) for details). An input-output model was estimated for each of these regions using the 1990 IMPLAN databases. The national average recreation spending profile was applied to each model (with 100,000 visits as level of recreation use), and the various aggregate multipliers were then calculated from the impact analysis. These multipliers therefore reflect the structural economic characteristics of each region. The aggregate multipliers are based on specific assumed recreation-spending profiles (Table 2), but can be applied generally to estimate the impacts of changes in recreation or tourism spending on the region.

The Type III multipliers were computed based on income rather than jobs to correct for a bias in the IMPLAN multiplier procedures. The induced effects estimated in the DOS version of IMPLAN (Version 91-F) assume an average salary associated with each job. As wages in recreation and tourism-related sectors are lower than average, this approach recirculates too much income as induced effects. Therefore, total income 
generated was computed as direct and indirect effects and applied to the model using the distribution for an average household (IMPLAN's medium PCE vector). The total induced effect is the sum of the infinite series of these rounds of respending of income. For recreation spending, the revised induced effects are about half of those estimated with the standard IMPLAN Type III procedures. The adjusted multipliers are comparable with those from the newer Windows version of IMPLAN when Type II multipliers are used.

The averages and ranges of the multipliers across the 108 regions are shown in Table 3. Multipliers for the local regions around each of the 108 projects are reported in Appendix C. These individual project multipliers were used to estimate impacts for the 108 projects in Groups One and Two. The averages reported in the top half of Table 3 were used for the 348 projects in Group Three.

\section{Table 3}

\section{Economic Multipliers for Regions Surrounding 108 CE Projects ${ }^{1}$}

\begin{tabular}{|c|c|c|c|}
\hline & Sales & Income ${ }^{2}$ & Jobs $^{3}$ \\
\hline \multicolumn{4}{|c|}{ Average Across 108 Regions } \\
\hline Direct effects & 1.00 & 0.52 & 33.27 \\
\hline Type I multiplier & 1.18 & 0.61 & 36.00 \\
\hline Type III multiplier & 1.66 & 0.87 & 44.71 \\
\hline Capture rate ${ }^{4}$ & 0.66 & & \\
\hline \multicolumn{4}{|c|}{ Range (minimum - maximum) } \\
\hline Direct effects & & $0.43-0.59$ & $19.87-46.89$ \\
\hline Type I multiplier & $1.09-1.28$ & $0.51-0.72$ & $22.73-49.66$ \\
\hline Type III multiplier & 1.37-1.99 & $0.66-1.11$ & $28.32-62.01$ \\
\hline Capture rate $^{4}$ & $0.53-0.83$ & & \\
\hline \multicolumn{4}{|c|}{$\begin{array}{l}1 \text { Region defined as all counties within } 48 \mathrm{~km} \text { ( } 30 \text { miles) project boundary. Multipliers are based on } \\
\text { IMPLAN-generated economic impact models constructed for } 108 \text { of the } 456 \text { projects (Becker } 1997) \text {. } \\
2 \text { Income per dollar of direct sales. Income includes employee compensation, proprietor, and other property income } \\
3 \text { Jobs per million dollars in direct sales. } \\
{ }^{4} \text { Capture rate is the percentage of visitor spending captured as direct sales within the region (see Appendix C). }\end{array}$} \\
\hline
\end{tabular}

The "average" project has a capture rate of 66 percent, meaning that 66 percent of visitor spending in the local region is captured locally as direct sales effects. Most of the "leakage" is visitor purchases of goods (e.g., gasoline, groceries, and souvenirs) that are not manufactured in the local area. Only the retail margins associated with these purchases generally accrue to the local economy. Capture rates varied from 53 to 83 percent, with most projects ranging between 60 and 70 percent.

Indirect effects associated with recreation spending were quite small and exhibited limited regional variation. The Type I sales multiplier captures indirect effects. The average project has a Type I sales multiplier of 1.18 , meaning that each dollar of direct sales generates an additional 18 cents in sales in industries that supply goods and services to tourism businesses (i.e., "backward linked" industries). The Type I sales multiplier 
varied only from 1.09 to 1.28 across the 108 projects. The Type III multiplier includes both indirect and induced effects. The average project has a Type III sales multiplier of 1.66 , meaning that each dollar of direct sales generates 18 cents in indirect sales and another 48 cents in induced effects. Induced effects stem from household spending of income earned from the direct and indirect effects. Type III sales multipliers vary somewhat more from a low of 1.4 to a high of 2.0 .

Income and job multipliers are used to convert direct sales to direct, indirect, and total income and employment effects. Type I income multipliers measure the total direct and indirect income associated with each dollar of direct sales. For an average project, each dollar of direct sales generates, in the local region, 52 cents in direct income, another 9 cents in indirect income $(0.61$ minus 0.52$)$ and 26 cents in induced income $(0.87$ minus 0.61). Employment multipliers are defined similarly but on the basis of the number of jobs per million dollars in direct sales. For an average project, each million dollars in direct sales supports about 33 direct jobs, 3 indirect jobs ( 36 minus 33), and 9 induced jobs (45 minus 36) for a total job impact of 45 jobs per million dollars of direct sales.

The range of variation across the 108 projects is larger for Type III multipliers than Type I. Employment multipliers exhibit significantly greater variation than income and sales multipliers. Thus, while one cannot go too far wrong using the average Type I sales multiplier of 1.18 (these vary only about 6 percent from the mean), the values of income multipliers can vary as much as 15 percent from the mean and the employment multipliers by over 30 percent. Capture rates and multipliers will vary systematically with the size and economic development of the region. Larger regions with more diversified economies will have higher capture rates and multipliers. Adjusting the average multipliers according to the general economic development of the surrounding region can therefore capture a great deal of the variation in multipliers across individual projects. It is recommended that managers of projects in Group Three use multipliers from a project in Group One or Two that has a similar level of local economic development (see Appendix C). 


\section{Results}

Estimates of visitation, spending, and economic impacts for all 456 projects are provided in Appendix B. A worksheet for computing these results is included in Appendix A. The worksheet has been completed using data for Mark Twain Lake. To estimate spending and impacts for any other project, one simply fills in the shaded cells (in some cases choosing to use the default values or to replace them) and completes the indicated calculations. The worksheet is also available as an Excel spreadsheet.

Individual project results are briefly interpreted below using Tables 4-7. These tables provide estimates of visits (Table 4), sales and spending (Table 5), income (Table 6) and employment for 36 selected projects. The results for all 456 projects are then aggregated into District, Division (Table 8), and national totals (Tables 9 and 10). 
Table 4

Visits by Segments for 36 Selected CE Projects

\begin{tabular}{|c|c|c|c|c|c|c|}
\hline \multirow[b]{3}{*}{ Method' } & \multirow[b]{3}{*}{ Project } & \multicolumn{5}{|c|}{ Visits in Party Days $(1,000 s)$} \\
\hline & & \multicolumn{2}{|c|}{ Campers } & \multicolumn{2}{|c|}{ Day Users ${ }^{2}$} & \multirow[b]{2}{*}{ Total } \\
\hline & & Boat & Nonboat & Boat & Nonboat & \\
\hline 1 & Cumberland & 35.24 & 30.68 & 894.88 & 819.36 & $1,780.15$ \\
\hline 1 & Dworshak & 0.98 & 1.79 & 14.36 & 27.53 & 44.66 \\
\hline 1 & J. Percy Priest & 3.39 & 18.85 & 331.08 & $1,937.09$ & $2,290.41$ \\
\hline 1 & McNary & 0.96 & 9.49 & 136.10 & $1,420.85$ & $1,567.40$ \\
\hline 1 & Mendocino & 8.53 & 29.68 & 60.33 & 220.85 & 319.38 \\
\hline 1 & Milford & 4.98 & 17.33 & 36.96 & 135.29 & 194.55 \\
\hline 1 & Oahe & 14.44 & 34.71 & 197.56 & 499.40 & 746.11 \\
\hline 1 & Ouachita & 15.83 & 44.22 & 118.71 & 348.84 & 527.60 \\
\hline 1 & Raystown & 10.84 & 28.77 & 80.71 & 225.30 & 345.62 \\
\hline 1 & Shelbyville & 17.77 & 74.39 & 152.37 & 670.69 & 915.23 \\
\hline 1 & Sidney Lanier & 41.35 & 82.43 & 813.02 & $1,704.33$ & $2,641.14$ \\
\hline 1 & Willamette Lakes ${ }^{3}$ & 5.31 & 11.61 & 178.49 & 410.19 & 605.61 \\
\hline 2 & Mark Twain Lake & 18.60 & 27.39 & 222.99 & 345.36 & 614.34 \\
\hline 2 & Harry S. Truman & 32.32 & 19.45 & 384.52 & 243.33 & 679.63 \\
\hline 2 & Blue Marsh Lake & 0.00 & 0.00 & 48.34 & 158.05 & 206.39 \\
\hline 2 & Saylorville Lake & 7.26 & 34.78 & 71.22 & 359.01 & 472.26 \\
\hline 2 & Lake Celilo & 1.14 & 3.01 & 107.22 & 299.30 & 410.67 \\
\hline 2 & Lower Granite & 3.23 & 10.61 & 82.46 & 285.05 & 381.35 \\
\hline 2 & Alum Creek Lake & 1.61 & 18.19 & 70.72 & 839.72 & 930.24 \\
\hline 2 & Wiiliam H. Harsha & 5.08 & 22.71 & 91.61 & 431.84 & 551.44 \\
\hline 2 & Allatoona Lake & 43.21 & 86.13 & 662.68 & $1,389.16$ & $2,181.18$ \\
\hline 2 & Whittier Narrows & 0.00 & 3.33 & 0.00 & 783.39 & 786.72 \\
\hline 2 & Belton Lake & 10.42 & 36.27 & 110.14 & 403.20 & 560.04 \\
\hline 2 & Murray & 2.86 & 22.73 & 42.37 & 353.94 & 421.89 \\
\hline 3 & Rivers Project - Illinois River & 0.00 & 0.00 & 33.33 & 63.90 & 97.23 \\
\hline 3 & Clinton Lake & 4.87 & 21.80 & 52.00 & 244.56 & 323.24 \\
\hline 3 & Black Rock Lake & 0.00 & 0.00 & 0.26 & 26.49 & 26.75 \\
\hline 3 & Almond Lake & 0.24 & 4.43 & 2.92 & 57.35 & 64.94 \\
\hline 3 & Duluth-Superior Harbor & 0.00 & 0.00 & 0.00 & 410.65 & 410.65 \\
\hline 3 & Blue River Lake & 0.00 & 0.00 & 3.64 & 15.03 & 18.67 \\
\hline 3 & Beech Fork Lake & 3.17 & 15.20 & 41.88 & 211.10 & 271.35 \\
\hline 3 & Brookville Lake & 5.10 & 24.42 & 66.85 & 336.98 & 433.35 \\
\hline 3 & Four River Basins & 0.00 & 0.00 & 15.35 & 63.41 & 78.77 \\
\hline 3 & Alamo Lâke & 2.56 & 0.75 & 16.92 & 5.22 & 25.44 \\
\hline 3 & Abiquiu Dam & 0.19 & 2.18 & 2.14 & 25.44 & 29.96 \\
\hline \multirow[t]{3}{*}{3} & Bardwell Lake & 3.15 & 10.37 & 30.01 & 103.72 & 147.25 \\
\hline & 456 Project Total & 1,438 & 4,925 & 28,650 & 104,405 & 139,419 \\
\hline & 456 Project Average & 3.15 & 10.80 & 62.83 & 228.96 & 305.74 \\
\hline $\begin{array}{l}{ }^{1} \text { Method } 1 \\
\text { of the } 108 \mathrm{p} \\
\text { use the nati } \\
{ }^{2} \text { Including } \\
{ }^{3} \text { Willamette } \\
12 \text {-lake sur }\end{array}$ & $\begin{array}{l}\text { s the } 12 \text { projects where surve } \\
\text { where the IMPLAN economi } \\
\text { gerage economic multipliers. } \\
\text { vernight visits. } \\
\text { include Fern Ridge Lake, Co } \\
\text { opst et al. 1992) for sampling }\end{array}$ & $\begin{array}{l}\text { used to create th } \\
\text { dels have been bi } \\
\text { Methods } 2 \text { and } \\
\text { Lake, and Fall } C\end{array}$ & $\begin{array}{l}\text { e spending profiles } \\
\text { uilt (Becker 1997); } \\
3 \text { were selected he } \\
\text { reek Lake. These t }\end{array}$ & $\begin{array}{l}\text { (Propst et al. } 199 \\
\text { Method } 3 \text { denotes } \\
\text { re to illustrate CE' } \\
\text { hree lakes were tr }\end{array}$ & $\begin{array}{l}\text { 2); both Methods } 1 \\
12 \text { of the other } 34 \\
\text { projects in differe } \\
\text { eated as a single p }\end{array}$ & $\begin{array}{l}\text { and } 2 \text { denote } 12 \\
8 \text { projects that } \\
\text { nt Divisions. } \\
\text { roject in the }\end{array}$ \\
\hline
\end{tabular}


Table 5

Regional Economic Impacts for 36 Selected CE Projects: Sales ${ }^{1}$

\begin{tabular}{|c|c|c|c|c|c|c|}
\hline \multirow[b]{2}{*}{ Method $^{2}$} & \multirow[b]{2}{*}{ Project } & \multirow{2}{*}{$\begin{array}{l}\text { Total (\$MM) } \\
\text { Spending }\end{array}$} & \multicolumn{4}{|c|}{ Sales Effects (\$MM) } \\
\hline & & & Direct & Indirect & Induced & Total \\
\hline 1 & Cumberland & 83.06 & 56.63 & 8.67 & 24.14 & 89.45 \\
\hline 1 & Dworshak & 1.93 & 1.11 & 0.11 & 0.30 & 1.52 \\
\hline 1 & J. Percy Priest & 87.33 & 60.29 & 15.02 & 37.95 & 113.27 \\
\hline 1 & McNary & 57.70 & 35.48 & 6.05 & 15.32 & 56.86 \\
\hline 1 & Mendocino & 13.14 & 8.31 & 1.47 & 4.48 & 14.27 \\
\hline 1 & Milford & 7.99 & 5.03 & 1.13 & 2.33 & 8.49 \\
\hline 1 & Oahe & 31.25 & 24.24 & 5.28 & 15.81 & 45.34 \\
\hline 1 & Ouachita & 22.10 & 13.95 & 2.36 & 8.76 & 25.07 \\
\hline 1 & Raystown & 14.55 & 9.19 & 1.38 & 4.60 & 15.16 \\
\hline 1 & Shelbyville & 36.83 & 23.27 & 3.77 & 8.16 & 35.21 \\
\hline 1 & Sidney Lanier & 112.19 & 74.98 & 14.98 & 40.60 & 130.56 \\
\hline 1 & Willamette Lakes ${ }^{3}$ & 25.31 & 16.23 & 2.25 & 9.59 & 28.07 \\
\hline 2 & Mark Twain Lake & 27.24 & 16.85 & 3.59 & 8.94 & 29.38 \\
\hline 2 & Harry S. Truman & 33.35 & 20.88 & 4.48 & 10.74 & 36.10 \\
\hline 2 & Blue Marsh Lake & 8.24 & 5.39 & 0.97 & 2.75 & 9.11 \\
\hline 2 & Saylorville Lake & 18.73 & 12.57 & 3.53 & 8.89 & 25.00 \\
\hline 2 & Lake Celilo & 16.72 & 10.37 & 1.58 & 4.31 & 16.27 \\
\hline 2 & Lower Granite & 15.33 & 9.51 & 1.60 & 5.17 & 16.28 \\
\hline 2 & Alum Creek Lake & 34.24 & 22.67 & 4.93 & 9.98 & 37.59 \\
\hline 2 & William H. Harsha & 21.69 & 14.76 & 3.55 & 8.02 & 26.34 \\
\hline 2 & Allatoona Lake & 93.01 & 62.55 & 12.91 & 22.08 & 97.54 \\
\hline 2 & Whittier Narrows & 27.43 & 22.70 & 5.67 & 12.45 & 40.82 \\
\hline 2 & Belton Lake & 22.76 & 14.05 & 2.28 & 5.88 & 22.21 \\
\hline 2 & Murray & 16.03 & 10.62 & 2.45 & 5.44 & 18.51 \\
\hline 3 & Rivers Project - Illinois River & 4.11 & 2.70 & 0.49 & 1.29 & 4.47 \\
\hline 3 & Clinton Lake & 12.86 & 8.43 & 1.52 & 4.02 & 13.97 \\
\hline 3 & Black Rock Lake & 0.94 & 0.61 & 0.11 & 0.29 & 1.02 \\
\hline 3 & Almond Lake & 2.40 & 1.57 & 0.28 & 0.75 & 2.60 \\
\hline 3 & Duluth-Superior Harbor & 14.29 & 9.37 & 1.69 & 4.47 & 15.53 \\
\hline 3 & Blue River Lake & 0.73 & 0.48 & 0.09 & 0.23 & 0.79 \\
\hline 3 & Beech Fork Lake & 10.68 & 7.01 & 1.26 & 3.34 & 11.61 \\
\hline 3 & Brookville Lake & 17.06 & 11.19 & 2.01 & 5.33 & 18.54 \\
\hline 3 & Four River Basins & 3.08 & 2.02 & 0.36 & 0.96 & 3.34 \\
\hline 3 & Alamo Lake & 1.35 & 0.88 & 0.16 & 0.42 & 1.46 \\
\hline 3 & Abiquiu Dam & 1.13 & 0.74 & 0.13 & 0.35 & 1.23 \\
\hline \multirow[t]{3}{*}{3} & Bardwell Lake & 6.03 & 3.96 & 0.71 & 1.89 & 6.55 \\
\hline & 456 Project Total & 5,596 & 3,691 & 679 & 1,777 & 6,148 \\
\hline & 456 Project Average & 12.27 & 8.10 & 1.49 & 3.90 & 13.48 \\
\hline \multicolumn{7}{|c|}{$\begin{array}{l}{ }^{1} \text { Impacts on counties within } 48 \mathrm{~km} \text { ( } 30 \text { miles) of CE projects by visitor trip spending within } 48 \mathrm{~km} \text { ( } 30 \text { miles) of the projects. } \\
{ }^{2} \text { Method } 1 \text { denotes the } 12 \text { projects where survey data were used to create the spending profiles (Propst et al. 1992); both Methods } 1 \text { and } 2 \text { denote } 12 \\
\text { of the } 108 \text { projects where the IMPLAN economic impact models have been built (Becker 1997); Method } 3 \text { denotes } 12 \text { of the other } 348 \text { projects that } \\
\text { use the national average economic multipliers. Projects from Methods } 2 \text { and } 3 \text { were selected here to illustrate CE's projects in different Divisions. } \\
{ }^{3} \text { Willamette Lakes include Fern Ridge Lake, Cottage Grove Lake, and Fall Creek Lake. These three lakes were treated as a single project in the } \\
\text { 12-lake survey (Propst et al. 1992) for sampling purposes. }\end{array}$} \\
\hline
\end{tabular}


Table 6

Regional Economic Impacts for 36 Selected CE Projects: Income ${ }^{1}$

\begin{tabular}{|c|c|c|c|c|c|}
\hline \multirow[b]{2}{*}{ Method $^{2}$} & \multirow[b]{2}{*}{ Project } & \multicolumn{4}{|c|}{ Income Effects (\$MM) } \\
\hline & & Direct & Indirect & Induced & Total \\
\hline 1. & Cumberland & 26.64 & 3.62 & 12.28 & 42.54 \\
\hline 1 & Dworshak & 0.58 & 0.06 & 0.17 & 0.81 \\
\hline 1 & J. Percy Priest & 32.69 & 8.10 & 20.98 & 61.77 \\
\hline 1 & McNary & 18.25 & 3.09 & 8.42 & 29.76 \\
\hline 1 & Mendocino & 4.56 & 0.84 & 2.54 & 7.94 \\
\hline 1 & Milford & 2.23 & 0.56 & 1.21 & 4.00 \\
\hline 1 & Oahe & 11.40 & 2.63 & 8.49 & 22.53 \\
\hline 1 & Ouachita & 7.39 & 1.26 & 4.78 & 13.43 \\
\hline 1 & Raystown & 4.96 & 0.67 & 2.50 & 8.13 \\
\hline 1 & Shelbyville & 11.30 & 1.98 & 4.39 & 17.67 \\
\hline 1 & Sidney Lanier & 43.16 & 8.41 & 23.00 & 74.57 \\
\hline 1 & Willamette Lakes ${ }^{3}$ & 9.49 & 1.14 & 5.41 & 16.04 \\
\hline 2 & Mark Twain Lake & 8.43 & 1.72 & 4.68 & 14.83 \\
\hline 2 & Harry S. Truman & 9.79 & 2.04 & 5.61 & 17.44 \\
\hline 2 & Blue Marsh Lake & 3.06 & 0.47 & 1.52 & 5.06 \\
\hline 2 & Saylorville Lake & 6.91 & 1.96 & 5.05 & 13.92 \\
\hline 2 & Lake Celilo & 5.89 & 0.81 & 2.47 & 9.17 \\
\hline 2 & Lower Granite & 4.70 & 0.85 & 2.87 & 8.42 \\
\hline 2 & Alum Creek Lake & 13.27 & 2.62 & 5.54 & 21.43 \\
\hline 2 & William H. Harsha & 8.64 & 2.00 & 4.54 & 15.18 \\
\hline 2 & Allatoona Lake & 36.62 & 7.34 & 12.84 & 56.80 \\
\hline 2 & Whittier Narrows & 12.24 & 3.29 & 7.15 & 22.68 \\
\hline 2 & Belton Lake & 7.42 & 1.26 & 3.34 & 12.01 \\
\hline 2 & Murray & 5.83 & 1.38 & 3.06 & 10.28 \\
\hline 3 & Rivers Project - Illinois River & 1.40 & 0.25 & 0.71 & 2.36 \\
\hline 3 & Clinton Lake & 4.37 & 0.79 & 2.21 & 7.38 \\
\hline 3 & Black Rock Lake & 0.32 & 0.06 & 0.16 & 0.54 \\
\hline 3 & Almond Lake & 0.81 & 0.15 & 0.41 & 1.37 \\
\hline 3 & Duluth-Superior Harbor & 4.86 & 0.88 & 2.46 & 8.20 \\
\hline 3 & Blue River Lake & 0.25 & 0.05 & 0.13 & 0.42 \\
\hline 3 & Beech Fork Lake & 3.63 & 0.66 & 1.84 & 6.13 \\
\hline 3 & Brookville Lake & 5.80 & 1.05 & 2.93 & 9.79 \\
\hline 3 & Four River Basins & 1.05 & 0.19 & 0.53 & 1.77 \\
\hline 3 & Alamo Lake & 0.46 & 0.08 & 0.23 & 0.77 \\
\hline 3 & Abiquiu Dam & 0.38 & 0.07 & 0.19 & 0.65 \\
\hline \multirow[t]{3}{*}{3} & Bardwell Lake & 2.05 & 0.37 & 1.04 & 3.46 \\
\hline & 456 Project Total & 1,914 & 355 & 975 & 3,244 \\
\hline & 456 Project Average & 4.20 & 0.78 & 2.14 & 7.11 \\
\hline \multicolumn{6}{|c|}{$\begin{array}{l}{ }^{1} \text { Impacts on counties within } 48 \mathrm{~km} \text { ( } 30 \text { miles) of CE projects by visitor trip spending within } 48 \mathrm{~km} \text { (30 miles) of the projects. Income includes employee } \\
\text { compensation, proprietor, and other property income. } \\
{ }^{2} \text { Method } 1 \text { denotes the } 12 \text { projects where survey data were used to create the spending profiles (Propst et al. 1992); both Methods } 1 \text { and } 2 \text { denote } 12 \\
\text { of the } 108 \text { projects where the IMPLAN economic impact models have been built (Becker 1997); Method } 3 \text { denotes } 12 \text { of the other } 348 \text { projects that } \\
\text { use the national average economic multipliers. Projects from Methods } 2 \text { and } 3 \text { were selected here to illustrate CE's projects in different Divisions. } \\
{ }^{3} \text { Willamette Lakes include Fern Ridge Lake, Cottage Grove Lake, and Fall Creek Lake. These three lakes were treated as a single project in the } \\
\text { 12-lake survey (Propst et al. 1992) for sampling purposes. }\end{array}$} \\
\hline
\end{tabular}


Table 7

Regional Economic Impacts for 36 Selected CE Projects: Jobs ${ }^{1}$

\begin{tabular}{|c|c|c|c|c|c|}
\hline \multirow[b]{2}{*}{ Method $^{2}$} & \multirow[b]{2}{*}{ Project } & \multicolumn{4}{|c|}{ Job Effects (number of jobs) } \\
\hline & & Direct & Indirect & Induced & Total \\
\hline 1 & Cumberland & 1,958 & 150 & 527 & 2,635 \\
\hline 1 & Dworshak & 44 & 2 & 7 & 54 \\
\hline 1 & J. Percy Priest & 1,649 & 216 & 606 & 2,471 \\
\hline 1 & McNary & 1,252 & 96 & 296 & 1,644 \\
\hline 1 & Mendocino & 267 & 24 & 77 & 367 \\
\hline 1 & Milford & 202 & 19 & 47 & 268 \\
\hline 1 & Oahe & 795 & 79 & 306 & 1,179 \\
\hline 1 & Ouachita & 515 & 41 & 180 & 737 \\
\hline 1 & Raystown & 345 & 22 & 83 & 450 \\
\hline 1 & Shelbyville & 773 & 49 & 154 & 976 \\
\hline 1 & Sidney Lanier & 2,054 & 202 & 657 & 2,913 \\
\hline 1 & Willamette Lakes ${ }^{3}$ & 587 & 29 & 181 & 797 \\
\hline 2 & Mark Twain Lake & 640 & 51 & 177 & 868 \\
\hline 2 & Harry S. Truman & 806 & 65 & 210 & 1,081 \\
\hline 2 & Blue Marsh Lake & 172 & 13 & 44 & 229 \\
\hline 2 & Saylorville Lake & 418 & 53 & 155 & 626 \\
\hline 2 & Lake Celilo & 379 & 24 & 87 & 490 \\
\hline 2 & Lower Granite & 389 & 28 & 110 & 526 \\
\hline 2 & Alum Creek Lake & 680 & 69 & 155 & 904 \\
\hline 2 & William H. Harsha & 427 & 50 & 128 & 605 \\
\hline 2 & Allatoona Lake & 1,636 & 164 & 338 & 2,138 \\
\hline 2 & Whittier Narrows & 451 & 65 & 157 & 673 \\
\hline 2 & Belton Lake & 510 & 39 & 106 & 655 \\
\hline 2 & Murray & 347 & 44 & 99 & 490 \\
\hline 3 & Rivers Project - Illinois River & 90 & 7 & 23 & 121 \\
\hline 3 & Clinton Lake & 281 & 23 & 73 & 377 \\
\hline 3 & Black Rock Lake & 20 & 2 & 5 & 27 \\
\hline 3 & Almond Lake & 52 & 4 & 14 & 70 \\
\hline 3 & Duluth-Superior Harbor & 312 & 26 & 82 & 419 \\
\hline 3 & Blue River Lake & 16 & 1 & 4 & 21 \\
\hline 3 & Beech Fork Lake & 233 & 19 & 61 & 313 \\
\hline 3 & Brookville Lake & 372 & 31 & 97 & 500 \\
\hline 3 & Four River Basins & 67 & 6 & 18 & 90 \\
\hline 3 & Alamo Lake & 29 & 2 & 8 & 39 \\
\hline 3 & Abiquiu Dam & 25 & 2 & 6 & 33 \\
\hline \multirow[t]{3}{*}{3} & Bardwell Lake & 132 & 11 & 34 & 177 \\
\hline & 456 Project Total & 120,906 & 10,242 & 32,499 & 163,647 \\
\hline & 456 Project Average & 265 & 22 & 71 & 359 \\
\hline \multicolumn{6}{|c|}{$\begin{array}{l}1 \text { Impacts on counties within } 48 \mathrm{~km} \text { ( } 30 \text { miles) of CE projects by visitor trip spending within } 48 \mathrm{~km} \text { ( } 30 \text { miles) of the projects. } \\
2 \text { Method } 1 \text { denotes the } 12 \text { projects where survey data were used to create the spending profiles (Propst et al. 1992); both Methods } 1 \text { and } 2 \text { denote } 12 \\
\text { of the } 108 \text { projects where the IMPLAN economic impact models have been built (Becker 1997); Method } 3 \text { denotes } 12 \text { of the other } 348 \text { projects that } \\
\text { use the national average economic multipliers. Projects from Methods } 2 \text { and } 3 \text { were selected here to illustrate CE's projects in different Divisions. } \\
{ }^{3} \text { Willamette Lakes include Fern Ridge Lake, Cottage Grove Lake, and Fall Creek Lake. These three lakes were treated as a single project in the } \\
\text { 12-lake survey (Propst et al. 1992) for sampling purposes. }\end{array}$} \\
\hline
\end{tabular}


Table 8

Summary Results for All CE Districts

\begin{tabular}{|c|c|c|c|c|c|c|c|c|c|c|c|}
\hline \multirow[b]{2}{*}{ Division } & \multirow[b]{2}{*}{ District } & \multicolumn{3}{|c|}{$\begin{array}{c}\text { Visits in Party Days } \\
(1,000 s)\end{array}$} & \multirow{2}{*}{$\begin{array}{l}\text { Total }{ }^{1} \\
\text { Spending }\end{array}$} & \multicolumn{2}{|c|}{ Sales Effects ${ }^{1}$} & \multicolumn{2}{|c|}{ Income Effects ${ }^{1}$} & \multicolumn{2}{|c|}{ Job Effects ${ }^{2}$} \\
\hline & & Camper & User $^{3}$ & Total & & Direct & Total & Direct & Total & Direct & Total \\
\hline \multirow{3}{*}{$\begin{array}{l}\text { Lower } \\
\text { Mississippi } \\
\text { Valley } \\
\text { (LMVD) } \\
\end{array}$} & St. Louis & 279 & 5,274 & 5,553 & 230 & 147 & 237 & 73 & 119 & 5,140 & 6,743 \\
\hline & \begin{tabular}{|l} 
Vicksburg \\
\end{tabular} & 191 & 3,640 & 3,832 & 158 & 101 & 160 & 51 & 82 & 3,550 & 4,658 \\
\hline & Subtotal & 470 & 8,914 & 9,385 & 388 & 248 & 397 & 124 & 201 & 8,690 & 11,401 \\
\hline \multirow{3}{*}{$\begin{array}{l}\text { Missouri } \\
\text { River (MRD) }\end{array}$} & Kansas City & 390 & 4,492 & 4,882 & 207 & 134 & 231 & 67 & 119 & 4,797 & 6,536 \\
\hline & Omaha & 313 & 4,850 & 5,163 & 203 & 138 & 234 & 71 & 123 & 4,494 & 6,170 \\
\hline & Subtotal & 703 & 9,342 & 10,045 & 410 & 272 & 465 & 138 & 242 & 9,291 & 12,706 \\
\hline \multirow{2}{*}{$\begin{array}{l}\text { New } \\
\text { England } \\
\text { (NED) }\end{array}$} & New England & 59 & 2,840 & 2,899 & 107 & 70 & 116 & 36 & 61 & 2,330 & 3,131 \\
\hline & Subtotal & 59 & 2,840 & 2,899 & 107 & 70 & 116 & 36 & 61 & 2,330 & 3,131 \\
\hline \multirow{4}{*}{$\begin{array}{l}\text { North } \\
\text { Atlantic } \\
\text { (NAD) }\end{array}$} & Baltimore & 96 & 678 & 774 & 32 & 21 & 35 & 11 & 18 & 733 & 972 \\
\hline & Norfolk & 0 & 119 & 119 & 4 & 3 & 5 & 1 & 2 & 95 & 127 \\
\hline & Philadelphia & 0 & 694 & 694 & 30 & 20 & 33 & 11 & 18 & 654 & 876 \\
\hline & Subtotal & 96 & 1,491 & 1,587 & 66 & 44 & 73 & 23 & 38 & 1,482 & 1,975 \\
\hline \multirow{4}{*}{$\begin{array}{l}\text { North } \\
\text { Central } \\
\text { (NCD) }\end{array}$} & Detroit & 0 & 722 & 722 & 25 & 17 & 27 & 9 & 15 & 552 & 742 \\
\hline & Rock Island & 206 & 6,285 & 6,491 & 248 & 163 & 275 & 85 & 146 & 5,429 & 7,360 \\
\hline & St. Paul & 86 & 3,500 & 3,586 & 170 & 111 & 184 & 58 & 97 & 3,704 & 4,977 \\
\hline & Subtotal & 292 & 10,507 & 10,799 & 443 & 291 & 486 & 152 & 258 & 9,685 & 13,079 \\
\hline \multirow{5}{*}{$\begin{array}{l}\text { North Pacific } \\
\text { (NPD) }\end{array}$} & Alaska & 6 & 47 & 53 & 2 & 1 & 2 & 1 & 1 & 43 & 58 \\
\hline & Portland & 68 & 4,116 & 4,183 & 168 & 109 & 176 & 58 & 95 & 3,652 & 4,804 \\
\hline & Seattle & 21 & 856 & 877 & 33 & 22 & 36 & 11 & 19 & 724 & 973 \\
\hline & Walla Walla & 58 & 2,636 & 2,695 & 103 & 65 & 105 & 33 & 55 & 2,301 & 3,053 \\
\hline & Subtotal & 153 & 7,655 & 7,808 & 306 & 197 & 319 & 103 & 170 & 6,720 & 8,888 \\
\hline \multirow{5}{*}{$\begin{array}{l}\text { Ohio River } \\
\text { (ORD) }\end{array}$} & Huntington & 392 & 8,773 & 9,165 & 342 & 224 & 370 & 118 & 198 & 7,379 & 9,910 \\
\hline & Louisville & 409 & 7,387 & 7,796 & 307 & 198 & 326 & 103 & 171 & 6,846 & 9,112 \\
\hline & Nashville & 307 & 14,363 & 14,670 & 587 & 386 & 649 & 196 & 336 & 12,474 & 17,102 \\
\hline & Pittsburgh & 114 & 2,227 & 2,341 & 94 & 62 & 103 & 32 & 55 & 2,067 & 2,792 \\
\hline & Subtotal & 1,222 & 32,750 & 33,972 & 1,330 & 870 & 1,448 & 449 & 760 & 28,766 & 38,916 \\
\hline \multirow{5}{*}{$\begin{array}{l}\text { South } \\
\text { Atlantic } \\
\text { (SAD) }\end{array}$} & Jacksonville & 192 & 2,413 & 2,605 & 106 & 69 & 115 & 36 & 61 & 2,309 & 3,103 \\
\hline & Mobile & 518 & 11,570 & 12,088 & 515 & 334 & 539 & 179 & 291 & 10,222 & 13,673 \\
\hline & Savannah & 320 & 6,649 & 6,970 & 285 & 188 & 311 & 98 & 165 & 6,030 & 8,159 \\
\hline & Wilmington & 279 & 3,593 & 3,872 & 159 & 99 & 152 & 51 & 80 & 3,418 & 4,354 \\
\hline & Subtotal & 1,309 & 24,225 & 25,535 & 1,065 & 690 & 1,117 & 364 & 597 & 21,979 & 29,289 \\
\hline \multirow{4}{*}{$\begin{array}{l}\text { South Pacific } \\
\text { (SPD) }\end{array}$} & Los Angeles & 14 & 2,643 & 2,657 & 93 & 73 & 129 & 39 & 71 & 1,661 & 2,397 \\
\hline & Sacramento & 128 & 1,182 & 1,310 & 55 & 35 & 60 & 19 & 33 & 1,118 & 1,517 \\
\hline & San Francisco & 0 & 44 & 44 & 2 & 1 & 2 & 1 & 1 & 34 & 46 \\
\hline & Subtotal & 142 & 3,869 & 4,011 & 150 & 109 & 191 & 59 & 105 & 2,813 & 3,960 \\
\hline \multirow{6}{*}{$\begin{array}{l}\text { Southwestern } \\
\text { (SWD) }\end{array}$} & Albuquerque & 24 & 324 & 348 & 13 & 9 & 14 & 5 & 8 & 290 & 389 \\
\hline & Fort Worth & 785 & 7,903 & 8,688 & 355 & 245 & 408 & 133 & 224 & 7,335 & 9,958 \\
\hline & Galveston & 0 & 1,295 & 1,295 & 45 & 33 & 54 & 18 & 30 & 905 & 1,220 \\
\hline & Little Rock & 502 & 13,288 & 13,790 & 561 & 365 & 644 & 188 & 337 & 12,814 & 18,073 \\
\hline & Tulsa & 606 & 8,649 & 9,255 & 355 & 250 & 415 & 125 & 214 & 7,806 & 10,660 \\
\hline & Subtotal & 1,917 & 31,459 & 33,376 & 1,329 & 902 & 1,535 & 469 & 813 & 29,150 & 40,300 \\
\hline \multicolumn{2}{|c|}{ All CE Project Total } & 6,363 & 133,052 & 139,417 & 5,594 & 3,693 & 6,147 & 1,917 & 3,245 & 120,906 & 163,645 \\
\hline
\end{tabular}


Table 9

Summary of Total CE Visits and Spending

\begin{tabular}{|c|c|c|c|c|c|c|c|}
\hline & \multicolumn{2}{|c|}{ Camper } & \multicolumn{2}{|c|}{ Day User } & \multicolumn{2}{|c|}{ Other Overnight } & \multirow[b]{2}{*}{ Total } \\
\hline & Boat & Nonboat & Boat & Nonboat & Boat & Nonboat & \\
\hline Visits (Party Days, MM) ${ }^{1}$ & 1.4 & 4.9 & 27.7 & 101.4 & 0.9 & 3.0 & 139.4 \\
\hline Percent of total & $1.0 \%$ & $3.5 \%$ & $19.9 \%$ & $72.7 \%$ & $0.7 \%$ & $2.2 \%$ & $100 \%$ \\
\hline Total spending in local regions $(\$ M M)^{2}$ & 94.8 & 243.9 & $1,504.8$ & $3,370.2$ & 118.5 & 263.6 & $5,595.8$ \\
\hline Percent of total & $1.7 \%$ & $4.4 \%$ & $26.9 \%$ & $60.2 \%$ & $2.1 \%$ & $4.7 \%$ & $100 \%$ \\
\hline
\end{tabular}

Table 10

Economic Impacts of Recreation Visitor Spending on 456 CE Projects

\begin{tabular}{|l|r|r|c||}
\hline & Sales (\$MM) & Income (\$MM) & Jobs (number of jobs) \\
\hline \hline Direct effects & 3,691 & 1,914 & 120,906 \\
\hline Indirect effects & 679 & 355 & 10,242 \\
\hline Induced effects & 1,777 & 975 & 32,499 \\
\hline \hline Total effects & 6,147 & 3,244 & 163,647 \\
\hline \hline
\end{tabular}

\section{Project Level Results}

Twelve projects have been selected from each of the three groups to illustrate the findings. The first 12 projects in Tables 4-7 (labeled Method 1) are the 12 lakes included in the 1989-90 visitor-spending surveys. Estimates for these projects are based on spending surveys at these 12 projects and multipliers generated from input-output models for the regions around each project (Appendix $C$ ). The second 12 projects (Method 2) include a project from each division for which multipliers were directly estimated (Appendix C). The spending profiles in Table 2 were assumed to apply to visitors at these projects. The final 12 projects (Method 3) include a project from each division for which both national spending averages (Table 2) and multiplier averages (Table 3 ) were used. The 1996 NMRS project-specific data used for all 456 projects were annual visits, camping revenue, number of CE-managed and non-CEmanaged campsites, and the percentage of visitors who were boating.

Mark Twain Lake serves to illustrate the interpretation of the findings, as well as the approach. Mark Twain Lake reported 1.636 million visits in 1996 with camping revenues of $\$ 297,864$. There were $440 \mathrm{CE}$-managed campsites at Mark Twain Lake and 103 non-CE-managed campsites. Boaters accounted for 40 percent of the visits. Based on these data from the 1996 NRMS database, Mark Twain hosted 614,340 party days of recreation use including 46,000 party nights of camping (Table 4). Only 3.4 percent of visitors to Mark Twain are camping on the project based on 
camping revenues. The NRMS figure for the percent of visitors camping at Mark Twain Lake is 10 percent. Recreation visitors to Mark Twain spent \$27.24 million in the local area in 1996 (Table 5).

Mark Twain Lake is illustrative of projects in Group Two, as projectspecific multipliers were estimated for the surrounding region using an input-output model. The multipliers for Mark Twain Lake are reported in Appendix B. Sixty-two percent of the $\$ 27.24$ million in visitor spending is captured as direct sales by the local economy $-\$ 16.85$ million in sales (Table 5). These direct sales generate another $\$ 3.59$ million in indirect sales and $\$ 8.94$ million in induced sales for a total sales effect of $\$ 29.38$ million. The Type I sales multiplier for Mark Twain is 1.21, computed as direct plus indirect sales divided by direct sales $((\$ 16.85+\$ 3.59) / \$ 16.85)$.

Income (Table 6) and employment (Table 7) impacts for Mark Twain are interpreted similarly. Visitor spending generates $\$ 8.43$ million in income and 640 jobs in sectors directly serving visitors. Another $\$ 1.72$ million in income and 51 jobs are created in backward-linked industries through indirect effects. Total impacts including direct, indirect, and induced effects of the $\$ 27.24$ million visitor spending are $\$ 29.38$ million in sales, $\$ 14.83$ million in income, and 868 jobs in the local region.

Mark Twain Lake is not the "average" project, as indicated by the multipliers and other model parameters. Mark Twain Lake has a higher than average percentage of campers and almost twice the percentage of boaters as an average project. Since campers and boaters spend more than day users and nonboaters, the project has a higher than average per-visitor spending (\$44 per party day compared with a $\$ 40$ per party-day average across all projects). The capture rate for Mark Twain is slightly lower than average, but slightly higher sales multipliers compensate for this difference. Income multipliers are slightly lower than average, while the employment multipliers are above average. This would indicate a region with lower than average wage rates. On balance, however, the impact results for Mark Twain would not be very different if the default average multipliers were used. In this case, the extra time and effort to estimate multipliers specific to this region do not substantially alter the results.

\section{Assumptions and Sources of Error}

The estimates for Mark Twain serve to illustrate the assumptions underlying the simplified approach and some of the potential sources of error. It is assumed that the visitation figures are correct as are the allocations of visits to various segments. There are likely errors in the camping share given the discrepancy between the revenue-based share and the percentage reported in NRMS (PR_USE). The assumption that 1 percent of day users are staying overnight in the area needs to be evaluated. If there are extensive overnight facilities on the project (other than camping) or in the 
surrounding area (including private campgrounds), the percentage of day visitors staying overnight may be substantially higher than 1 percent. Spending and impacts would then also be higher. The reported 40 percent of visitors boating is much higher than average and, if incorrect, could also be a source of error in the spending and impact estimates.

The approach assumes that the average segment spending profiles in Table 2 adequately represent Mark Twain Lake visitors. Errors in the spending estimates will occur if Mark Twain Lake visitors tend to spend considerably more or less than the averages in Table 2 . If so, the spending figures should be adjusted on the worksheet. Since the multipliers are directly estimated from an input-output model for the Mark Twain region, they should be reasonably accurate. Readers should be aware however that multipliers can vary by as much as 10 percent across different models for the same region (e.g., a model estimated with IMPLAN versus a RIMS II model), so that some error in the multipliers is inevitable when a complex input-output model is used.

The economic impact results reported here are what Jackson et al. (1992) have called a "significance analysis." That is, they include the impacts of spending by both local residents and visitors from outside the local region. A strict "impact analysis" would only include the spending of nonresidents, as this spending represents "new" dollars to the region's economy. All of the local region's economic activity associated with visitor spending would not necessarily be lost if recreation was not provided at Mark Twain Lake. The economic activity reported in Tables 4-7 and Appendix B should therefore be interpreted as economic activity associated with recreation on Mark Twain Lake. This activity is a measure of the significance or importance of the project to the local economy. It is fairly straightforward to adapt the worksheet to carry out a true impact analysis (i.e., including only nonresident spending), but this requires good information on the percentage of visitors within each segment that come from outside the local region. At this time, most CE projects have only a rough idea of the percentage of day users and campers coming from outside the local region.

\section{District and Division Impacts}

The availability of visitation, spending, and economic impact estimates for all $456 \mathrm{CE}$ projects makes it a simple task to generate economic impacts for CE Districts and Divisions. Division and District totals are reported in Table 8 . It should be noted that these are simply aggregations of the local impacts of individual projects in each District or Division. The findings do not therefore cover all impacts on the larger region, but only the sum of the impacts on local areas around projects in a given District or Division. A complete estimate of impacts for the larger regions would need to include visitor spending outside local areas surrounding each project and should employ multipliers for the larger region. 
Approximately two-thirds of the income and employment effects occur in 3 of the Corps' 10 Divisions. Fifty-five percent of these effects occur in 7 of the Corps' 31 Districts. Comparisons of the relative impacts of projects in each District or Division should take into account the demographic and economic characteristics of the regions. For example, a thousand jobs in a District with relatively low population and economic activity have a much greater relative impact than a thousand jobs in a heavily populated, economically diverse District. Nonetheless, District and Division personnel may find these comparisons useful, especially when compared with the economic impacts of other industries (e.g., agriculture or manufacturing) in a given District or Division.

\section{National Economic Effects}

The aggregation may be taken one step further to the national level, with the same caveats as above. Multiplying visitation at all 456 projects (Table 1 ) by average spending (Table 2 ) yields a total of $\$ 5.6$ billion in trip-related expenditures associated with recreational use of CE projects in 1996 (Table 9). These are trip expenditures only within $48 \mathrm{~km}$ (30 miles) of CE projects and not total trip expenses. Day users account for 87 percent of this total. Other overnight visitors account for 7 percent of total spending as compared with 6 percent for campers. The other overnight estimate is based on a somewhat arbitrary estimate that 1 percent of day-use visitors are staying overnight in the area. Boaters account for 22 percent of party days on CE projects and 31 percent of all spending.

In 1996, the $\$ 5.6$ billion in visitor spending associated with the CE recreation program resulted in direct effects of approximately $\$ 1.9$ billion in income and 121,000 jobs within the counties around CE projects (Table 10). When secondary effects are considered, the local economic effects of $\mathrm{CE}$ visitor spending totals over $\$ 3.2$ billion in income and 164,000 jobs. It is important to recall that these impacts are the result of spending by CE visitors locally (within $48 \mathrm{~km}$ (30 miles) of a project's borders) and employ local area multipliers, not national ones. Total effects represent slightly more than 0.1 percent of total U.S. jobs and income. In terms of secondary impacts, induced effects dominate indirect effects by about 3 to 1 . This reflects the labor-intensive nature of the tourism industry. 


\section{Errors in Applying the Simplified Approach}

As a prelude to recommendations for refining these estimates and updating them on a regular basis, potential errors involved in the suggested approach need to be assessed and acknowledged. Discussion of errors in economic impact estimates is quite rare. Although regional economic analysts are well aware of a wide range of potential errors in their estimates, these errors are difficult to quantify, given the number of assumptions and judgements that are involved in the models. There is a tendency to assume that methods based on subjective judgements and simple models are prone to larger errors than those based on complex models and primary data gathering. This is not necessarily the case. The degree of error depends on the quality of the subjective judgements versus the validity of model assumptions and accuracy of estimates from statistical samples. Both spending surveys and regional economic modeling efforts are subject to potentially large errors if the studies are not carefully conducted. There are also cases where a manager's or researcher's judgements about visitor numbers or characteristics have proven to be far off the mark.

Errors in estimates of economic impacts can arise from each of the three factors in Equation 1. In applying the simplified approach, errors are largely due to generalizing a national average or value from a different project or region to the particular application. These errors can be reduced considerably by revising or replacing the default spending and/or multiplier values based on sound judgement or better local information about visitors. Project managers can use the worksheet in Appendix A to refine or update the spending and impact estimates for individual projects.

\section{Visitation}

The economic impact results are sensitive to the number of visits as well as estimates of the proportion of visits across distinct visitor segments. Visitation figures should be accurate, although there are questions about how or whether to include visitors who are not engaged in any 
specific activity at the project. These "sightseers" can represent a fairly high percentage of use at some projects. For impact estimates, the issue is whether the day-use spending profiles adequately represent sightseers and more substantively whether sightseer spending and associated impacts should be attributed to the project.

The proportion of campers and other overnight visitors staying off the project are important, as these segments spend significantly more than day users. Taking into account length of stay, a typical camper party spends over $\$ 150$ in the local region during the stay, compared with about $\$ 40$ for a typical day-use party. Visitors staying overnight in the area will also spend substantially more than day users.

$\mathrm{CE}$ data on the numbers of campers and other overnight visitors are less reliable than the overall visitation numbers. The estimates of the percentage of visitors that are camping on each project varied considerably depending on which source of data was used. Using camping revenues, 2 percent of $C E$ visitors nationally were estimated to be camping. The percent based on the "percent camping" reported in the NRMS (PR_USE) database was 8 percent. Camping estimates are even more variable for individual projects, suggesting that camping estimates can be off by over 400 percent, depending on which estimate is used and which is correct. For example, the camping percentage for Mark Twain Lake in the NRMS database is 10 percent, while the revenue-based estimate is only 3.4 percent. Using the 10-percent figure at Mark Twain Lake would increase the visitor spending estimate by 13 percent, from $\$ 27$ million to $\$ 31$ million. Thus, large errors are possible if accurate visitation figures are not fed into the NRMS. This potential for error underscores the need for local project staff to be involved in identifying questionable results and providing more accurate information when available.

Projects do not have reliable information about the number of visitors staying overnight in the area off CE project lands. The "other overnight" segments should also include visitors staying in motels, lodges, and cabins on the project. These visitors spend three times what a day user spends on a per day basis and about 10 times as much on a per visit (trip) basis. Lacking good data on overnight visitors, 1 percent of day users were assumed to be staying overnight in the area. Each 1,000 additional day users who are staying overnight off the project increases visitor spending by $\$ 80,000$. Thus, a change from 1 to 2 percent for other overnight visitors would increase spending by $\$ 80,000$ at a project with 100,000 day users.

The percentage of visitors estimated to be boating also influences the spending and impact estimates, as boaters spend from 30 to 60 percent more than nonboaters. For example, if the percent boating is incorrectly estimated at 10 percent when it is actually 20 percent, total spending would be underestimated by about 5 percent. 
The overall estimate of visits is the most important parameter, as the estimates of the number of visitors and party days within each segment are derived as proportions of this total. Average party-size and length-ofstay parameters will also introduce potential errors, as these are used in converting visits to a party-day basis. Taking all of these potential sources of error into account, the speculation is that errors in the estimates of the percentages of visitors from each segment will introduce errors in the spending and impact estimates in the range of 5-15 percent. If there are large numbers of day users staying overnight that are not properly accounted for, the errors can be much greater. Large errors in estimating the total number of visitors would, of course, also produce significant errors in the spending and impact estimates.

\section{Spending Averages}

The spending averages come from surveys of visitors and are, therefore, subject to sampling and other errors. Sampling errors for the 12-lake averages for individual segments are generally between 5 and 20 percent (95-percent confidence intervals). Fortunately, the spending estimates are most reliable for the largest segments - day users and campers. Sampling errors in the survey estimates of spending can therefore introduce errors of about 10 percent in total spending estimates; however, these are not the errors that are the biggest concern.

When used to estimate impacts for a particular project, the issue is how well the spending averages reflect visitors to that project. Some idea of the potential error in generalizing the segment spending profiles to individual projects can be gleaned from the range of variation across the 12 lakes included in the spending survey. Segment spending averages for these individual projects generally fell within 33 percent of the 12-lake averages. As some of this variation is likely due to sampling errors (given small samples within segments at individual projects), the use of the national averages at individual projects will likely introduce errors of less than \pm 25 percent for most projects. Projects with unique spending patterns should not use the national averages. For example, spending by overnight visitors that were boating at Lake Cumberland were more than double the national average due to a large proportion of houseboat rentals. Other projects with significant onsite commercial development will also experience higher rates of spending.

\section{Multipliers}

Across 108 projects for which multipliers were estimated, the capture rates varied, from an average of 66 percent, by less than \pm 10 percent for most local regions. Type I sales multipliers exhibit even less variation, 
ranging by about \pm 6 percent around the mean of 1.18 . Type III multipliers and income and employment ratios vary more widely around their means, although most of this variation is predictable based on the degree of economic development of the region. The Type I income multiplier and Type III sales multipliers vary by \pm 15 percent, while the employment multipliers vary by as much as 35 percent from the mean across the 108 regions.

Applying the average multiplier values to individual projects will generally contribute errors of less than 10 percent in estimating the direct and indirect sales effects. Errors can be slightly larger for the direct and indirect income effects, and can be as high as 20 percent in estimating induced effects. Employment ratios and multipliers exhibit even greater variation, and the averages should therefore be used with some caution. Projects for which multipliers are not reported in Appendix C should replace the averages in the worksheet with multipliers from a similar region to refine the impact estimates.

Since any errors in estimates of the direct effects will be multiplied by these multipliers, obtaining accurate estimates of visits and spending first is of paramount importance. The direct effects are by definition more directly associated with recreation on CE projects and therefore deserve the most attention.

\section{Cumulative Effect of Errors}

It is difficult to estimate the cumulative effect of all the sources of error. This is because the cumulative effect depends on whether the errors in each factor (visitation, spending, and multipliers) are in opposite directions and "cancel" each other or whether they simply "add up." Since input-output analysis is linear (the larger the inputs, the larger the outputs), errors of 100 percent in visitation estimates will result in similar errors in spending and impact estimates. At present, it is not possible to estimate the error associated with total visitation in the NRMS. However, a source of potentially large error in the estimate of the percentage of campers was detected. Therefore, most effort should be directed at obtaining accurate total visitation estimates as well as proportions of campers, day users, boaters, and other visitor segments. 


\section{Conclusions and Recommendations}

The economic effects of visitor spending associated with recreational use of CE projects can be estimated using data that are regularly gathered and reported in the NRMS databases. The accuracy of these estimates depends on several factors: accuracy of the recreation-use estimates, proper allocation of total use to visitor segments, applicability of the spending profiles for each visitor segment, and the appropriateness of the regional economic multipliers for the surrounding region. The highest priorities for improving the accuracy of spending and impact estimates would appear to be improvements in the recording of visitation data and conducting additional spending surveys. The analysis and findings in this report suggest a number of ways to improve the CE estimates of use and spending.

a. Data on campsite occupancy, generally available at the project level, should be added to the NRMS database. Reliable data on the number of camping party nights (occupied site nights) is more directly useful for estimating camper spending than camping revenue or the percentage of visitors who are camping. Revenue data are confounded by distinct fees and charges. Estimating campers as a percentage of all users creates large errors when survey estimates of this percentage are applied to the large number of visitors. The most useful information for campers is the number of party nights or equivalently occupied sites. These data should also be available for non-CE-managed campgrounds.

$b$. Surveys should estimate the proportion of CE day users staying overnight off the project in motels, campgrounds, etc. These visitors have different spending patterns than day users and can have significant impacts on the local economy. Overnight stays in lodges, motels, and cabins on CE projects should also be separated from day users and included in the "other overnight" category.

c. New surveys should be conducted to update the spending profiles and provide averages for a wider range of projects. The spending data used here are 8 years old. While price indices perform quite well in 
updating the spending averages, averages for a wider range of projects are needed to better understand how spending averages vary across projects. Attempts to develop models to explain variations in spending across projects have been unsuccessful, in part due to small samples at only a small number of projects. Becker (1977) provides recommendations for projects that would be good candidates to sample in future expenditure surveys.

d. Multipliers seem to be of somewhat lower priority in terms of their contribution to error. Multipliers can vary by as much as 10 percent between different models for the same region (e.g., between an IMPLAN and RIMS II model), so errors of 10 percent or less in the multipliers should be expected. The Type I multipliers from IMPLAN exhibit fairly limited variation across the regions around $\mathrm{CE}$ projects. With readily available input-output modeling packages like IMPLAN, one can obtain multipliers for any local region at little expense. Errors due to multipliers are more likely to come from misuse than estimation errors.

The economic impacts estimated in this report may be used to assess the $\mathrm{CE}$ recreation program at the project, District, or Division levels. The results may be used to evaluate the potential economic effects of natural resource allocation and management decisions affecting recreation opportunities at all three levels. For example, given acceptance of the visitor spending profiles and multipliers presented herein, individuals may conduct "what if" analyses. The likely impacts of a change in recreation use can be estimated using the worksheet in Appendix A. Instead of entering total annual use, one would enter the change in the number and types of visitors. Spending averages and other parameters can also be adjusted to reflect the policy or action being evaluated.

The worksheet in Appendix A is provided so that project managers can also refine and update the impact estimates for their project. The underlying Excel spreadsheet for these computations may be downloaded from the WWW (http://www/msu.edu/user/stynes/usace). The spending and impact estimates may be refined by replacing the default values for any of the variables in the spreadsheet (shaded cells) with better estimates for a given project. The impact estimates can also be easily updated by substituting visitation data in future years. The spreadsheet version of the worksheet includes price indices for updating the spending averages over time and other tips for choosing appropriate values for each cell.

CE projects vary considerably in project characteristics, levels and types of recreation activity, and in terms of the nature of the economy of the surrounding region. Visitor spending and its impacts on the local economy depend on all of these factors. The simplified economic impact procedures presented herein attempt to capture as many of these factors as possible to explain variations in spending and associated impacts at different projects. The procedures rely entirely on available data, while also suggesting new data gathering and reporting procedures that could improve 
the estimates. The simplified impact estimation procedure is designed to give quick aggregate estimates of economic impacts at the project level. When more detailed estimates are needed and when time and resources permit, the MI-REC system should be used. 


\section{References}

Alward, G. S., and Palmer, C. J. (1983). "IMPLAN: An input-output analysis system for Forest Service planning." Forest sector models, Proceedings of the First North American Conference on Forest Sector Modeling (Williamsburg, VA). R. Seppala, C. Row, and A. Morgan, ed., A B Academic Publishing, Oxford, 131-140.

Becker, D. R. (1997). "Classification of Corps of Engineers projects for economic impact assessment," M.S. thesis, Michigan State University, Department of Park, Recreation and Tourism Resources, East Lansing, MI.

Carlson, B. D., Propst, D. B., Stynes, D. J., and Jackson, R. S. (1995). "Economic impact of recreation on the Upper Mississippi River System," Technical Report EL-95-16, U.S. Army Engineer Waterways Experiment Station, Vicksburg, MS.

Jackson, R. S., Stynes, D. J., and Propst, D. B. (1994). “An assessment of the national economic effects of the U.S. Army Corps of Engineers recreation program," Miscellaneous Paper R-94-2, U.S. Army Engineer Waterways Experiment Station, Vicksburg, MS.

Jackson, R. S., Stynes, D. J., Propst, D. B., and Carlson, B. D. (1996). “A summary of the national and state economic effects of the 1994 U.S. Army Corps of Engineers Recreation Program," Technical Report R-96-1, U.S. Army Engineer Waterways Experiment Station, Vicksburg, MS.

Jackson, R. S., Stynes, D. J., Propst, D. B., and Siverts, E. (1992). "Economic impacts analysis as a tool in recreation program evaluation," Instruction Report R-92-1, U.S. Army Engineer Waterways Experiment Station, Vicksburg, MS.

Propst, D. B., Stynes, D. J., Lee, J. H., and Jackson, R. S. (1992). "Development of spending profiles for recreation visitors to Corps of Engineers projects," Technical Report R-92-4, U.S. Army Engineer Waterways Experiment Station, Vicksburg, MS. 
Propst, D. B., Stynes, D. J., and Rutz, E. A. (1995a). "Economic impacts of recreation at Lake Cumberland, Kentucky," Department of Park, Recreation and Tourism Resources, Michigan State University, East Lansing, MI.

. (1995b). "Economic impacts of recreation at Dworshak

Reservoir, Idaho," Department of Park, Recreation and Tourism Resources, Michigan State University, East Lansing, MI.

. (1995c). "Economic impacts of recreation at McNary Lock

\& Dam (Lake Wallula), Oregon/Washington," Department of Park, Recreation and Tourism Resources, Michigan State University, East Lansing, MI.

. (1995d). "Economic impacts of recreation at Raystown Lake, Pennsylvania," Department of Park, Recreation and Tourism Resources, Michigan State University, East Lansing, MI.

- (1995e). "Economic impacts of recreation at Lake Shelbyville, Illinois," Department of Park, Recreation and Tourism Resources, Michigan State University, East Lansing, MI.

. (1995f). "Economic impacts of recreation at the Willamette Lakes, Oregon," Department of Park, Recreation and Tourism Resources, Michigan State University, East Lansing, MI.

Stynes, D. J., and Propst, D. B. (1996). MI-REC 2.0 User's Manual. Department of Park, Recreation and Tourism Resources, Michigan State University and Michigan State University Agricultural Experiment Station, East Lansing, MI.

Stynes, D. J, Propst, D. B., and Rutz, E. A. (1995g). "Economic impacts of recreation at J. Percy Priest Reservoir, Tennessee," Department of Park, Recreation and Tourism Resources, Michigan State University, East Lansing, MI.

. (1995h). "Economic impacts of recreation at Lake Mendocino, California," Department of Park, Recreation and Tourism Resources, Michigan State University, East Lansing, MI.

. (1995i). "Economic impacts of recreation at Milford Lake, Kansas," Department of Park, Recreation and Tourism Resources, Michigan State University, East Lansing, MI.

. (1995j). "Economic impacts of recreation at Oahe Reservoir, North Dakota/South Dakota," Department of Park, Recreation and Tourism Resources, Michigan State University, East Lansing, MI. 
Stynes, D. J, Propst, D. B., and Rutz, E. A. (1995k). "Economic impacts of recreation at Lake Ouachita, Arkansas," Department of Park, Recreation and Tourism Resources, Michigan State University, East Lansing, MI.

. (19951). "Economic impacts of recreation at Lake Sidney

Lanier, Georgia," Department of Park, Recreation and Tourism

Resources, Michigan State University, East Lansing, MI.

Taylor, C., Winter, S., Alward, G., and Siverts, E. (1993). Micro IMPLAN User's Guide. U.S. Department of Agriculture, Land Management Planning Systems Group, Fort Collins, CO.

U.S. Army Corps of Engineers. (1996). Natural Resource Management System (NRMS), Washington, DC.

U.S. Department of Interior. (1990). The Money Generation Model. Office of Social Science, Socio-economic Studies Division, National Park Service, Denver, CO. 
Appendix A

Worksheet for Estimating

Economic Impacts of Visitor

Spending at Corps of Engineers

(CE) Projects 


\section{CE Economic Impact Estimator}

Line 1

Line 2

Line 3

Line 4

Line 5

Line 6

Line 7

Line 8

Line 9

Line 10

Line 11

Line 12

Line 13

Line 14

Line 15

Line 16

Line 17

Line 18

Line 19

Line 20 sites

Enter average revenue per site

3. Determine day use visits project

15) segment (person visits)

6. Enter party size and length of stay factors
1. Enter number of visits to the project

2. Determine the number of camper party nights at $C E$

Enter total camping revenue (CE sites)

Camper party nights $=$ Line $2 \div$ Line 3

3. Add campers at non-CE-managed sites

Enter number of CE-managed sites

Enter number of non-CE-managed sites

Expansion factor (Line 5 + Line 6) $\div$ Line 5)

Total camper party nights (Line $7 \times$ Line 4 )

4. Convert campers to person visits

Enter average camper length of stay

Camper party visits $=$ Line $8 \div$ Line 9

Enter average camping party size

Camper person visits $=$ Line $10 \times$ Line 11

Percent of visitors camping (Line $12 \div$ Line 1 )

Day use person visits $=$ Line $1-$ Line 12

4. Estimate number of "other overnight" visitors

Enter pct of day users staying overnight off project

Enter pct of day users staying overnight on the

Number of overnight off-project visits (Line $14 \times$ Line

Pure day users (Line 14 - Line 16)

5. Estimate number of boaters and nonboaters in each

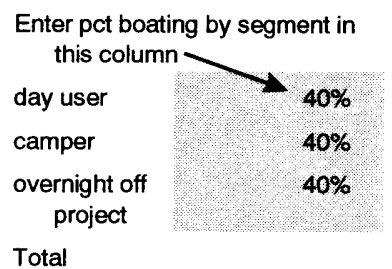

Boaters
626,002
22,318
6,323

Nonboaters

Total

939,002

33,477

9,485

$1,565,004$

55,795

15,808

654,643

981,964

$1,636,607$

\begin{tabular}{|c|c|c|c|c|c|}
\hline & & Boaters & Nonboaters & Boaters & Nonboaters \\
\hline & day user & 2.90 & 280 & 1.0 & 10 \\
\hline & camper & 3.60 & 3.30 & 3.0 & 2.7 \\
\hline & overnight off & 3.37 & 294 & 3.8 & 3.1 \\
\hline & \multicolumn{5}{|c|}{ 7. Convert to party days/nights by setment. (person visits $\times$ length of stay $\div$ party size) } \\
\hline \multirow[t]{6}{*}{ Line 20} & & Boaters & Nonboaters & Total & Percent \\
\hline & day user & $215,862.59$ & $335,357.95$ & 551,221 & $90 \%$ \\
\hline & camper & $18,598.37$ & $27,390.32$ & 45,989 & $7 \%$ \\
\hline & $\begin{array}{l}\text { overnight off } \\
\text { project }\end{array}$ & $7,130.07$ & $10,001.05$ & 17,131 & $3 \%$ \\
\hline & Total & 241,591 & 372,749 & 614,340 & $100 \%$ \\
\hline & Percent & $39 \%$ & $61 \%$ & $100 \%$ & \\
\hline
\end{tabular}


8. Enter/edit segment spending averages (per party night)

\begin{tabular}{|c|c|c|c|c|}
\hline & \multicolumn{2}{|c|}{ Spending within 30 miles } & \multicolumn{2}{|c|}{ Default values } \\
\hline & Boaters & Nonboaters & Boaters & Nonboaters \\
\hline day user & $\$ 54.26$ & $\$ 33.24$ & $\$ 54.26$ & $\$ 33.24$ \\
\hline camper & $\$ 65.93$ & $\$ 49.52$ & $\$ 65.93$ & $\$ 49.52$ \\
\hline $\begin{array}{l}\text { overnight off } \\
\text { project }\end{array}$ & $\$ 129.36$ & $\$ 87.17$ & $\$ 129.36$ & $\$ 87.17$ \\
\hline
\end{tabular}

Line 22

9. Compute total spending (\$MM's) (per party night spending times number of party nights) Spending within 30 miles (\$MM)

\begin{tabular}{lrrrr} 
& Boaters & Nonboaters & \multicolumn{1}{c}{ Total } & Percent \\
day user & $\$ 11.71$ & $\$ 11.15$ & $\$ 22.86$ & $84 \%$ \\
camper & $\$ 1.23$ & $\$ 1.36$ & $\$ 2.58$ & $9 \%$ \\
$\begin{array}{c}\text { overnight off } \\
\text { project }\end{array}$ & $\$ 0.92$ & $\$ 0.87$ & $\$ 1.79$ & $7 \%$ \\
Total & $\$ 13.86$ & $\$ 13.38$ & $\$ 27.24$ & $100 \%$
\end{tabular}

Line $23 \quad$ 10. Enter multipliers for the region

Capture rate

$49 \%$

$100 \%$

Type I sales

Type III sales

Income/sales direct effects

Income/Sales Type I

Income/Sales III

Jobs/Sales direct

Job/Sales 1

Job/Sales III

\begin{tabular}{|cc} 
Mark Twain Lake & Defauts \\
\hline $62 \%$ & $66 \%$ \\
1.21 & 1.18 \\
1.74 & 1.66 \\
$50 \%$ & $52 \%$ \\
$60 \%$ & $61 \%$ \\
$88 \%$ & $87 \%$ \\
38.00 & 33.27 \\
41.01 & 36.00 \\
51.52 & 44.71
\end{tabular}

Line 24

11. Compute Direct, Indirect, Induced, and Total Effects

\begin{tabular}{|c|c|c|c|}
\hline & Sales (SMM) & Income (SMM) & Jobs \\
\hline Direct Effects & $\$ 16.85$ & $\$ 8.43$ & 640 \\
\hline Indirect Effects & $\$ 3.59$ & $\$ 1.72$ & 51 \\
\hline Induced Effects & $\$ 8.94$ & $\$ 4.68$ & 177 \\
\hline Total Effects & $\$ 29.38$ & $\$ 14.83$ & 868 \\
\hline Person Visits & $1,686,607$ & & \\
\hline Party Days/nights & 614,340 & & \\
\hline Visitor Spending (\$MM) & $\$ 27.24$ & & \\
\hline
\end{tabular}

Formulas for Line 24

Direct sales $=$ total visitor spending $\times$ capture rate

Direct income $=$ direct sales $\times$ direct income $\div$ sales ratio

Direct jobs $=$ direct sales $\times$ direct jobs $\div$ sales ratio

Indirect sales $=$ direct sales $\times($ Type I sales multiplier -1$)$

Induced sales $=$ direct sales $\times$ (Type III sales mult. - Type I sales mult.)

Total sales $=$ direct sales $\times$ Type III sales multiplier $=$ direct + indirect + induced effects

Indirect income $=$ direct sales $\times$ Type $\mathrm{I}$ income multiplier - direct income

Induced income $=$ direct sales $\times$ Type III income multiplier - direct income - indirect income

Total income $=$ direct sales $\times$ Type III income multiplier $=$ direct + indirect + induced income

Indirect jobs $=$ direct sales $\times$ Type $\mathrm{I}$ jobs multiplier - direct jobs

Induced jobs $=$ direct sales $\times$ Type III jobs multiplier - direct jobs - indirect jobs

Total jobs $=$ direct sales $\times$ Type III jobs multiplier $=$ direct + indirect + induced jobs 


\section{Worksheet Notes by Line Number}

Line 1

Line 2

Line 3

Line 4

Line 5

Line 6

Line 7

Line 8

Line 9

Line 10

Line 11

Line 12

Line 13

Line 14

Line 15

Line 16

Line 17

Line 18

Line 19

Line 21

Line 22

Line 23

ine 24

\section{Other Notes}

1. One can easily evaluate the sensitivity of results to any of the worksheet parameters by changing one or more cells and observing the effect on total spending or impact measures. Advanced Excel users can also make use of Excel's built in "What if" analysis tools.

2. This initial version of the worksheet does not fully account for visitors staying in lodges, cabins, or motels on the project. Look for updated versions of this worksheet that more fully capture spending of overnight visitors at our website.

Field VISITS from PR_USE NRMS database. (in person visits)

Field CG_FEE_REV from CUR_FEE database, includes all camping revenue at CE-managed sites

This should reflect the average fee paid per night per site. The $\$ 8$ default is an estimate taking into account variation between sites with or without electricity, and discounts for Golden passport and access programs.

the number of camping party nights is known, it can be directly entered on Line 4 and then Lines 2 and 3 are unnecessary.

Include all campsites covered by the camping fees reported on Line 2.

Any campsites on the project not covered by the camping fees reported on Line 2 should be included. This factor expands use from CE-managed campsites to all sites. Lines 5-7 are then not needed.

Substitute a local value for the average length of stay by campers, if available.

Substitute a local value for the average number of people per camping party, if available.

A person visit is one person entering project. Campers should be counted only once during their stay. This is the percent of visitors (person visits) that are camping.

There are two groups of "other overnight visitors": those staying overnight off project in the local area and visitors staying in lodges, motels, cabins, and other accommodations (except campgrounds) on the project. Enter each as a percentage of day use visits.

Pure day users are not staying overnight in the local area (unless they live there)

for all visitors in the NRMS. The percentage may be varied across the three segments here, if differences are known. Otherwise enter the NRMS value in all three cells. 1.0. This avoids double counting of spending by those who make multiple visits during their stay.

Replace or adjust spending averages to suit local conditions or based on a local survey.

Default multipliers may be pasted in or replaced by values from a similar region. See Appendix $C$ to find multipliers for $108 \mathrm{CE}$ projects. For projects not listed, use multipliers for projects in a similar regional economic setting. 


\section{Appendix B \\ Summary Results for All Corps of Engineers Projects}




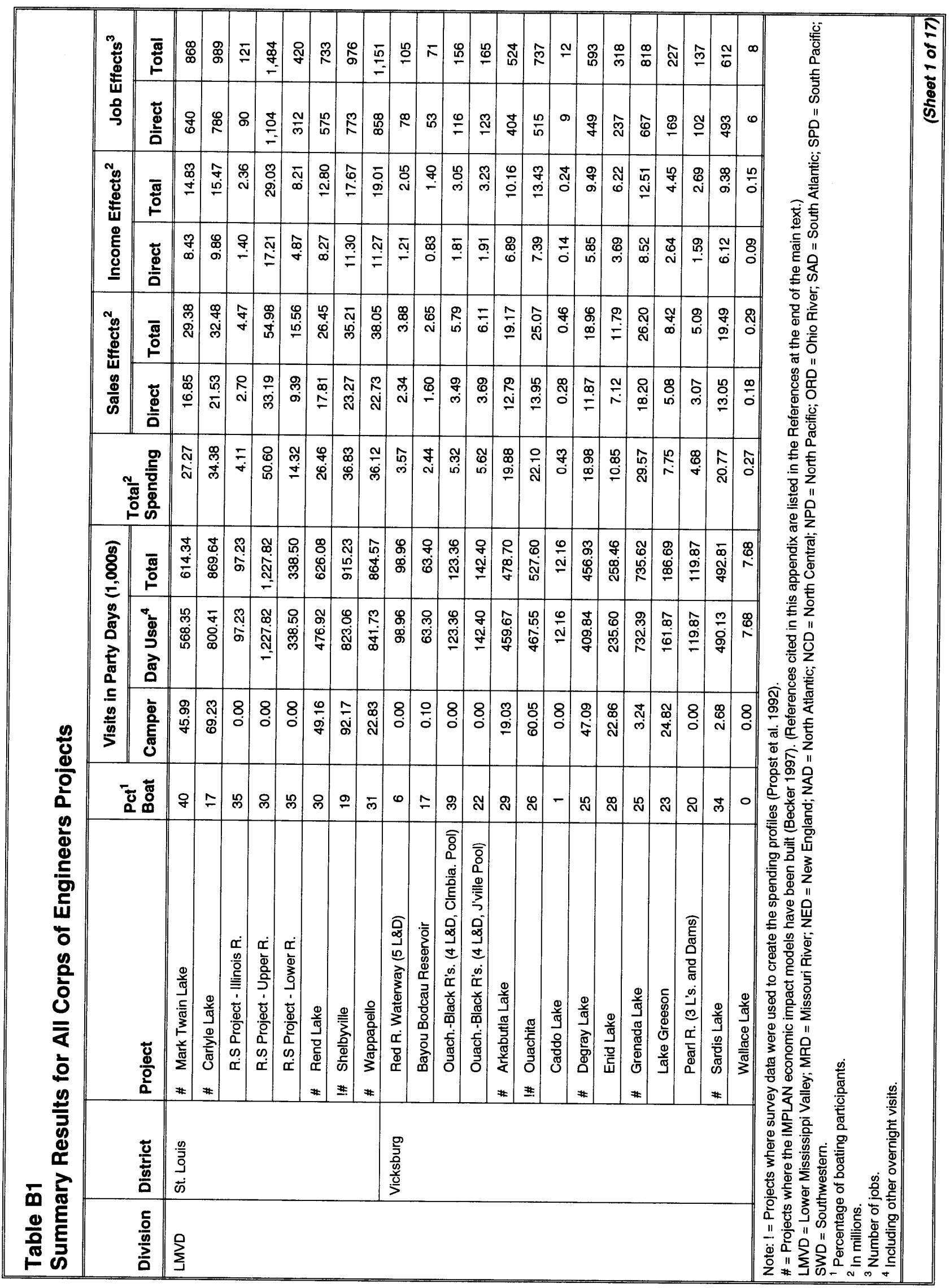




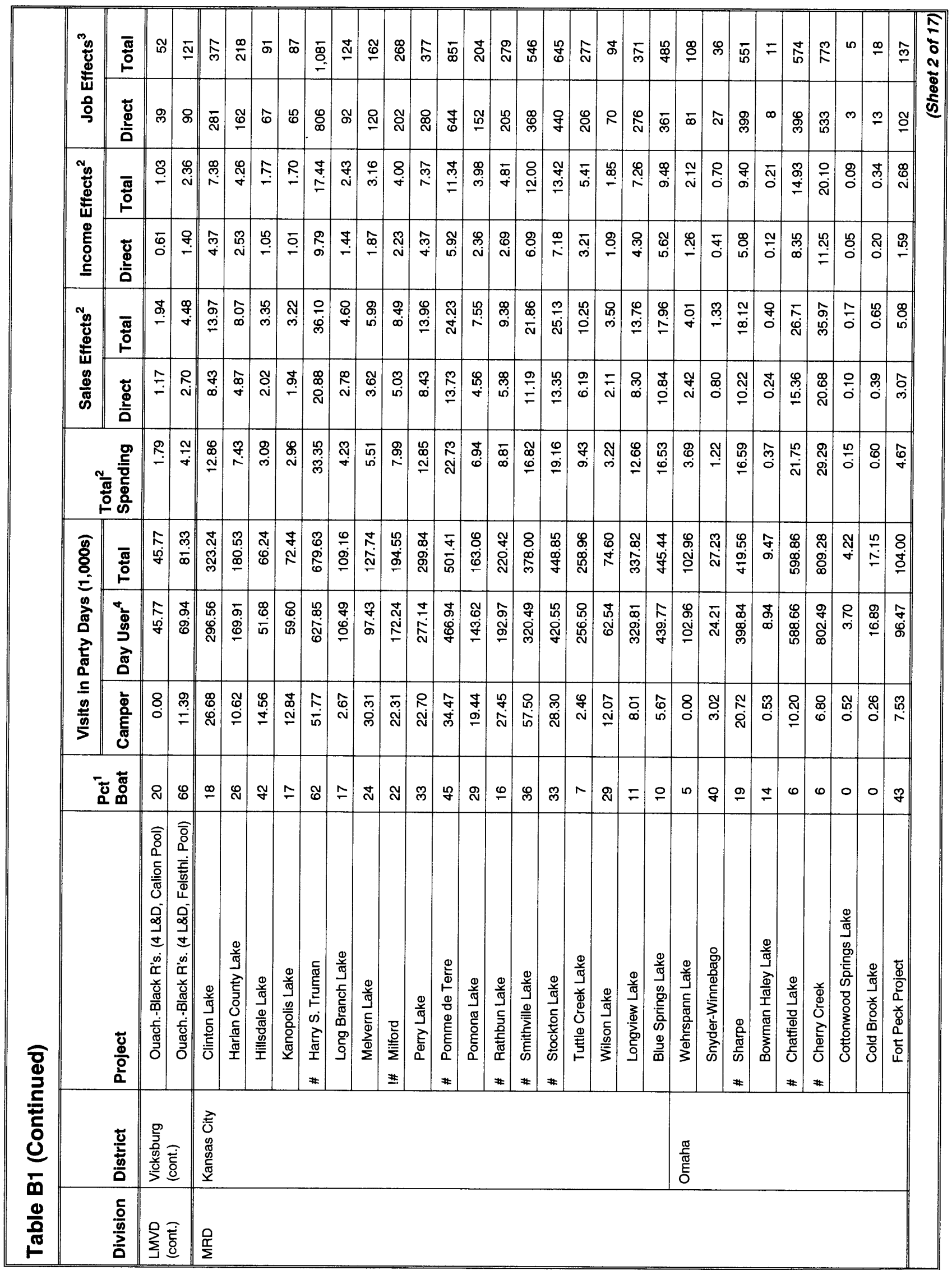




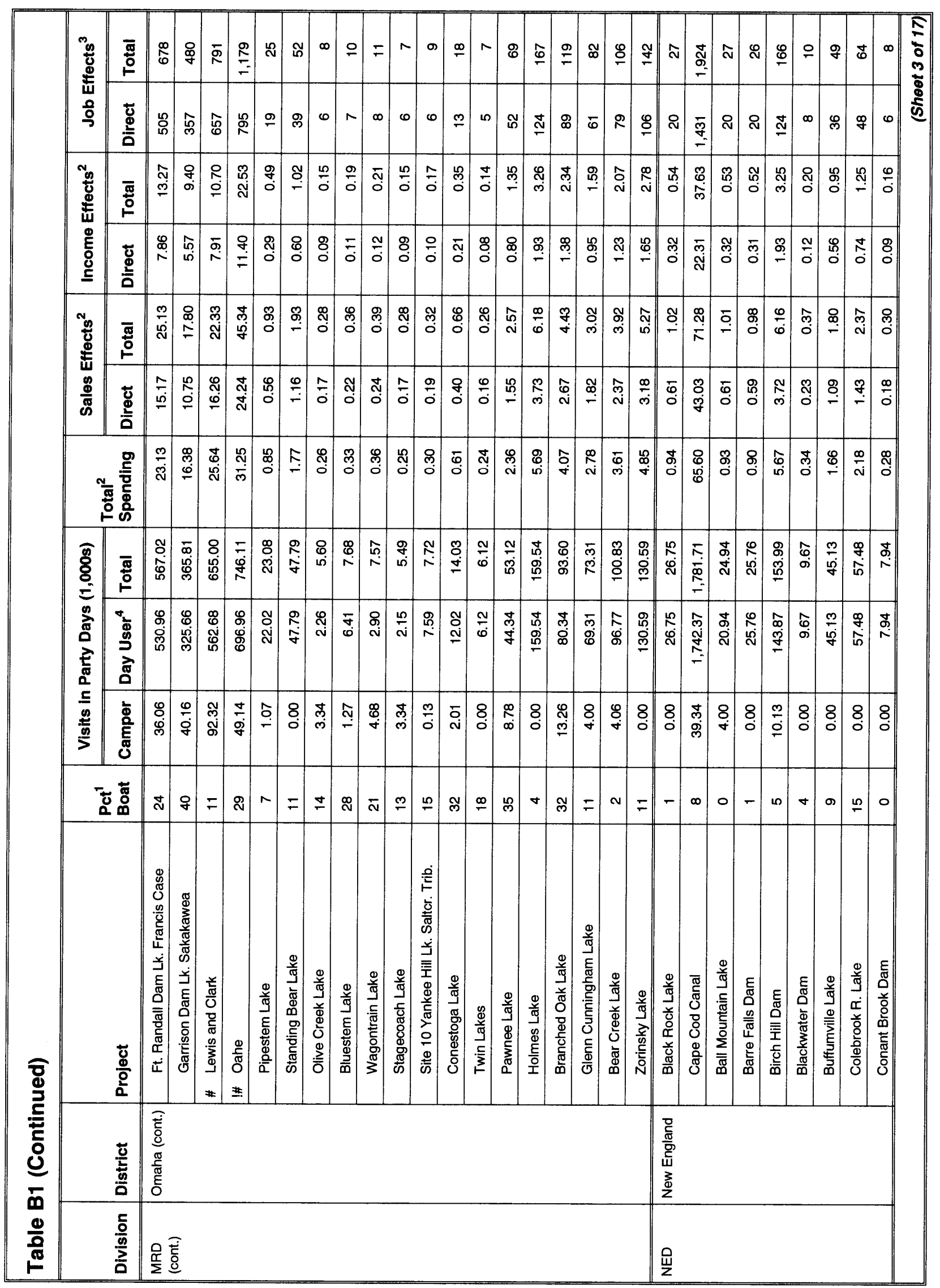




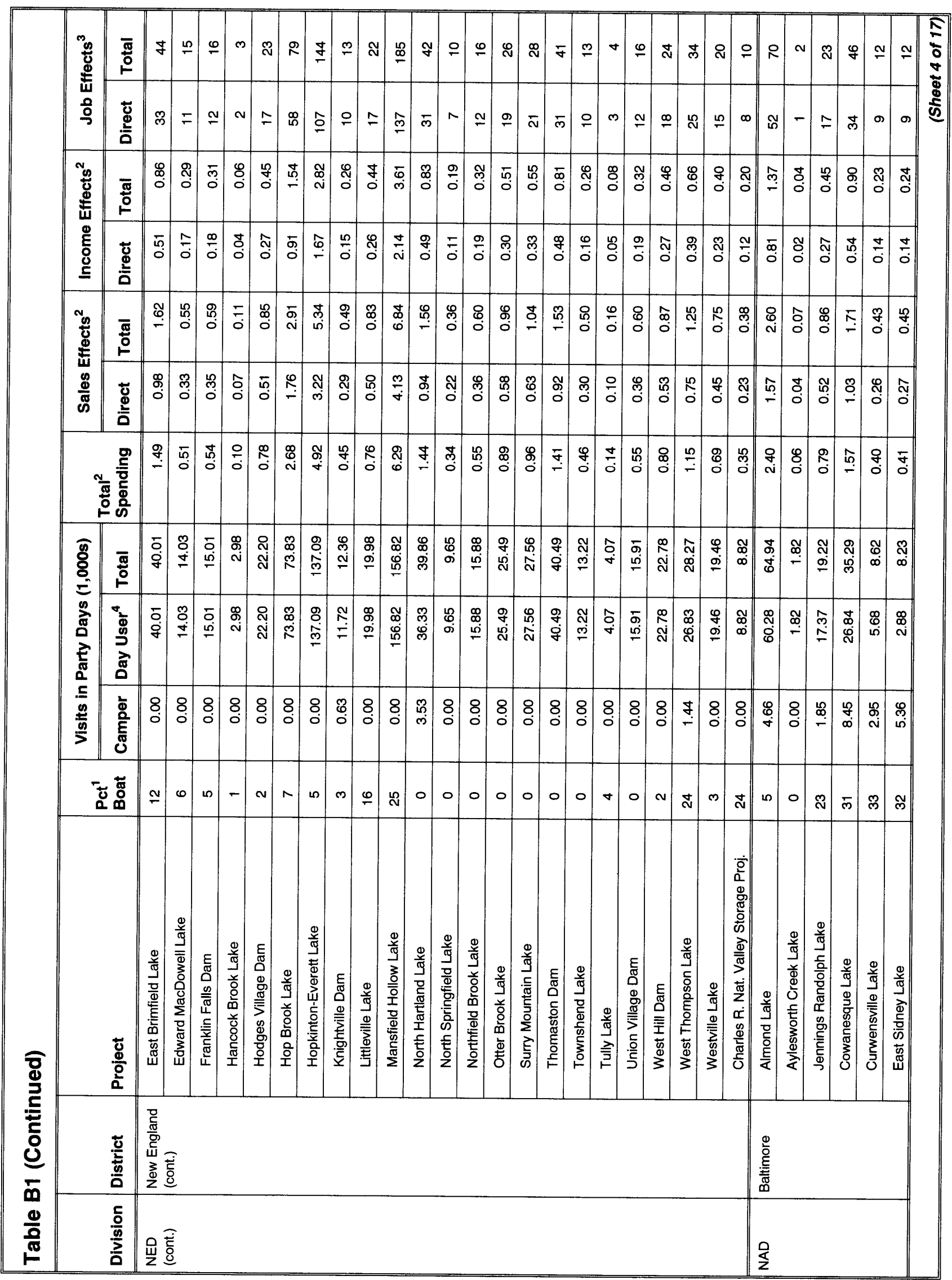




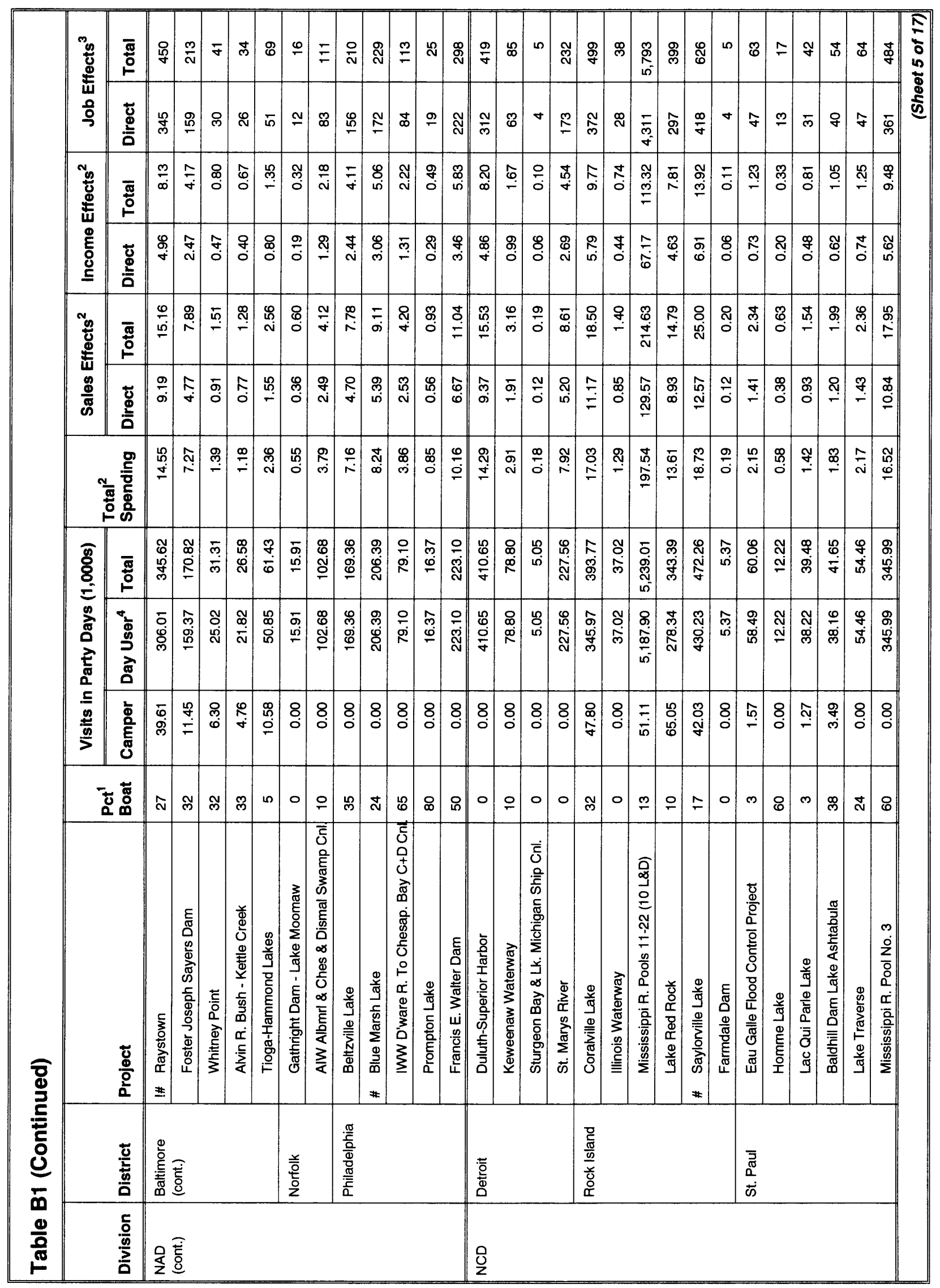




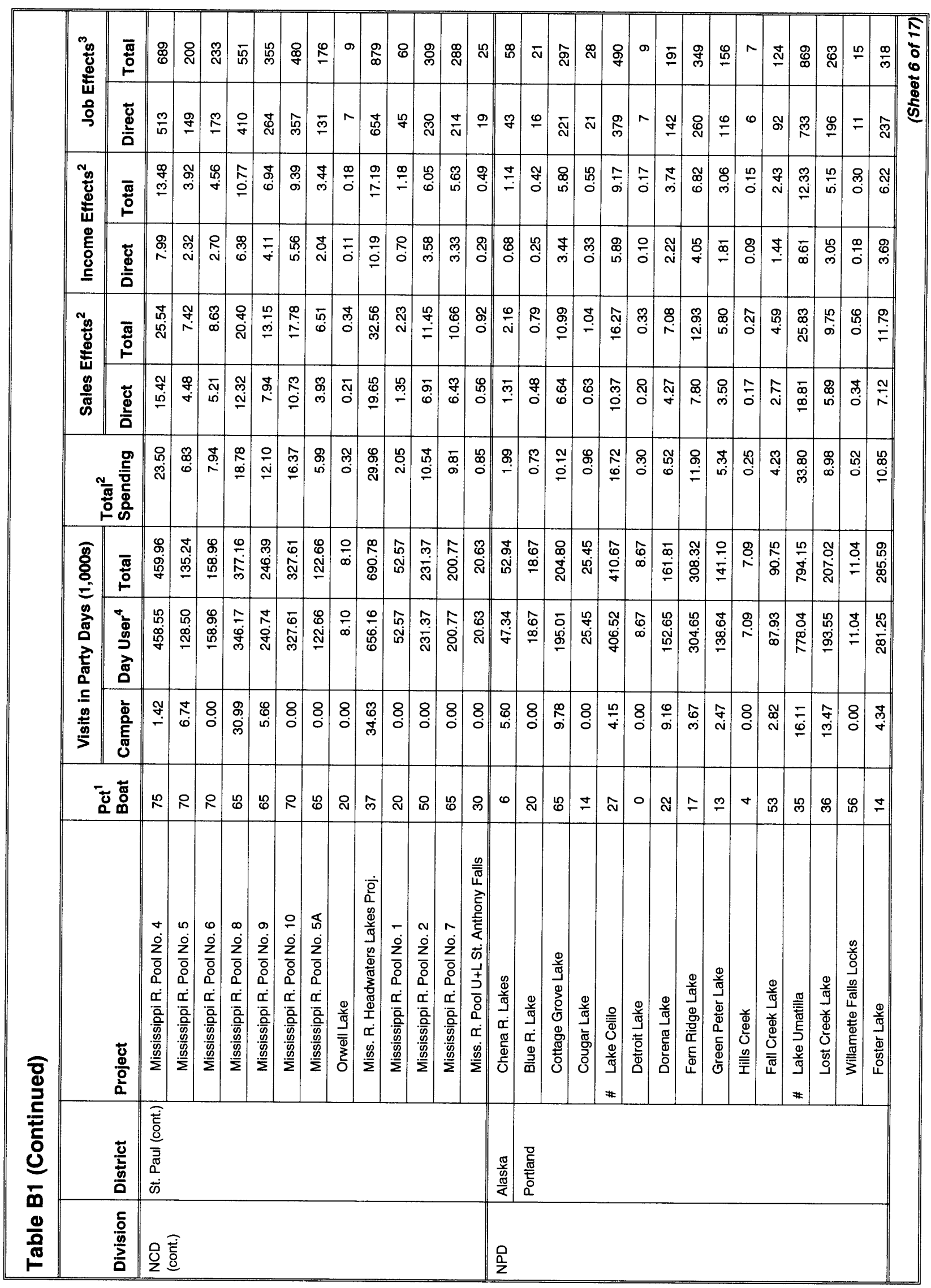




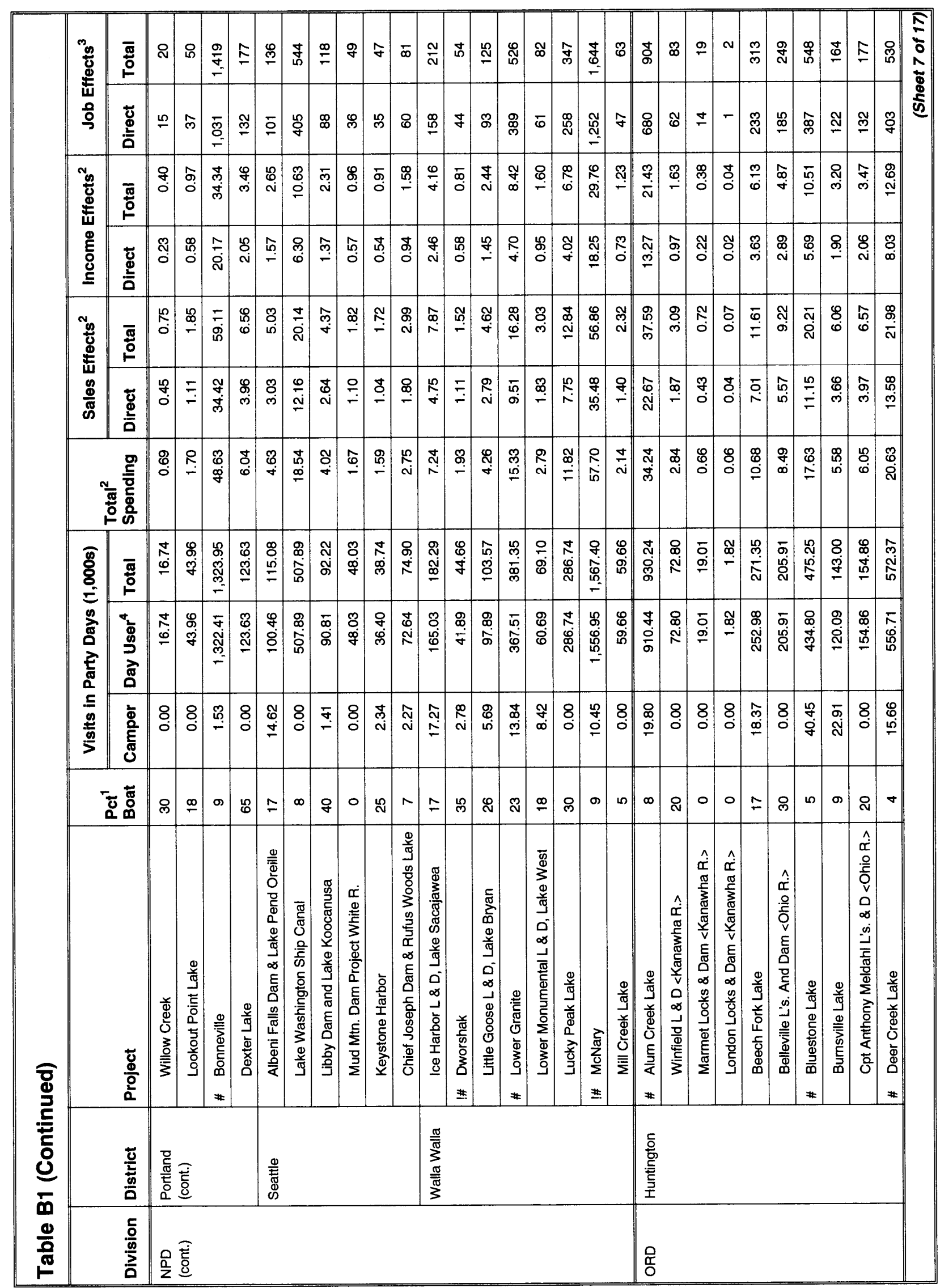




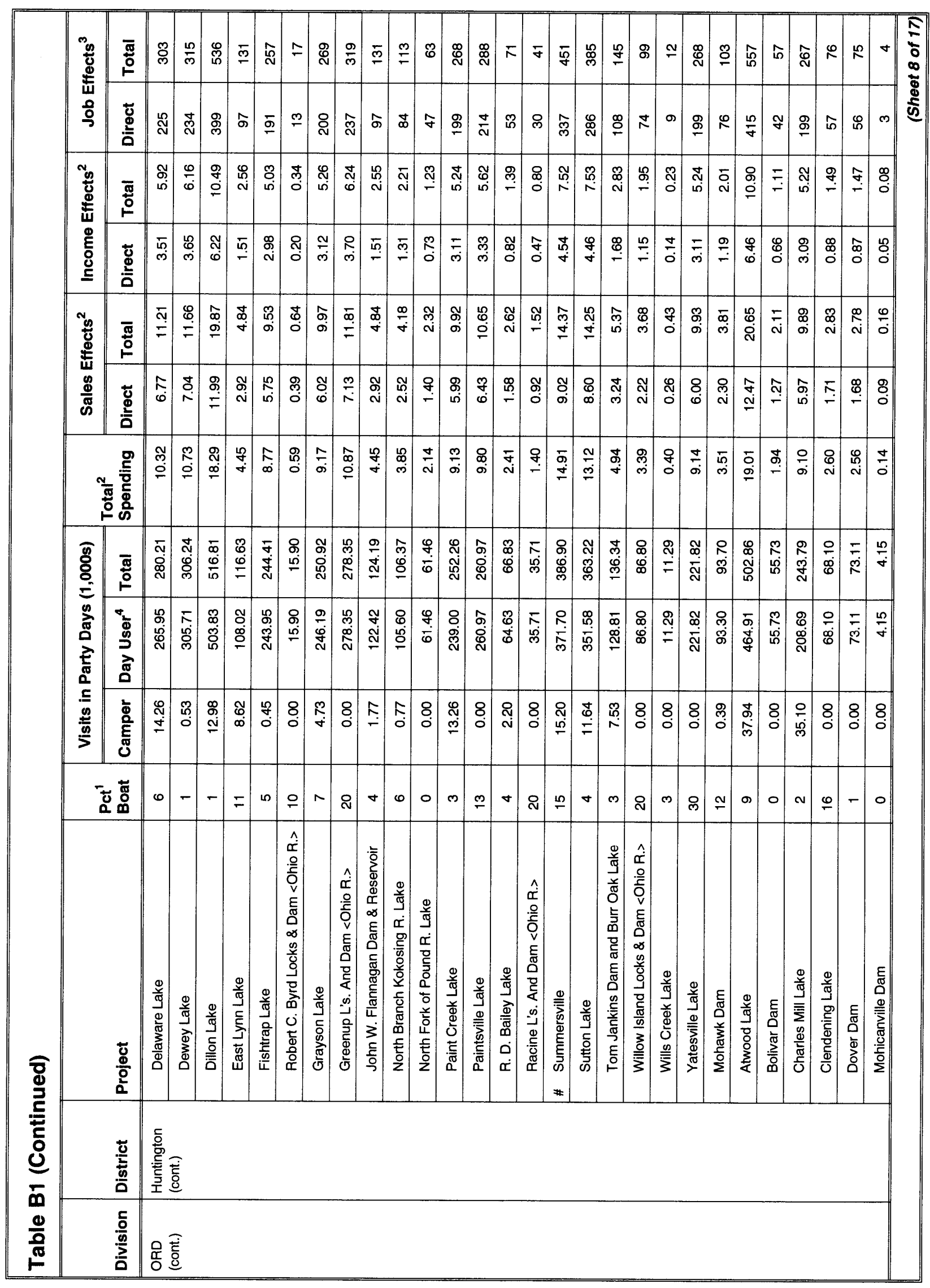




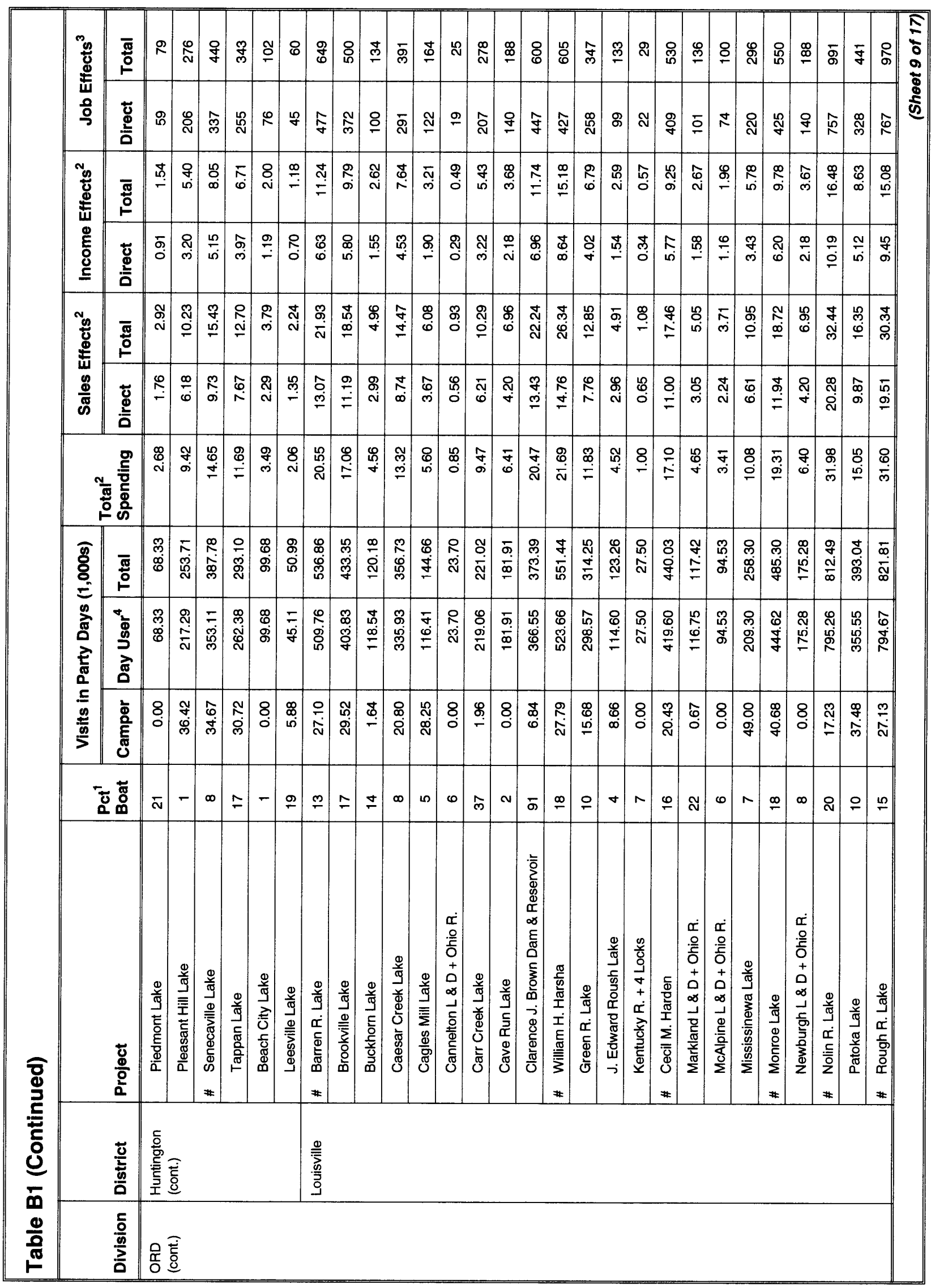




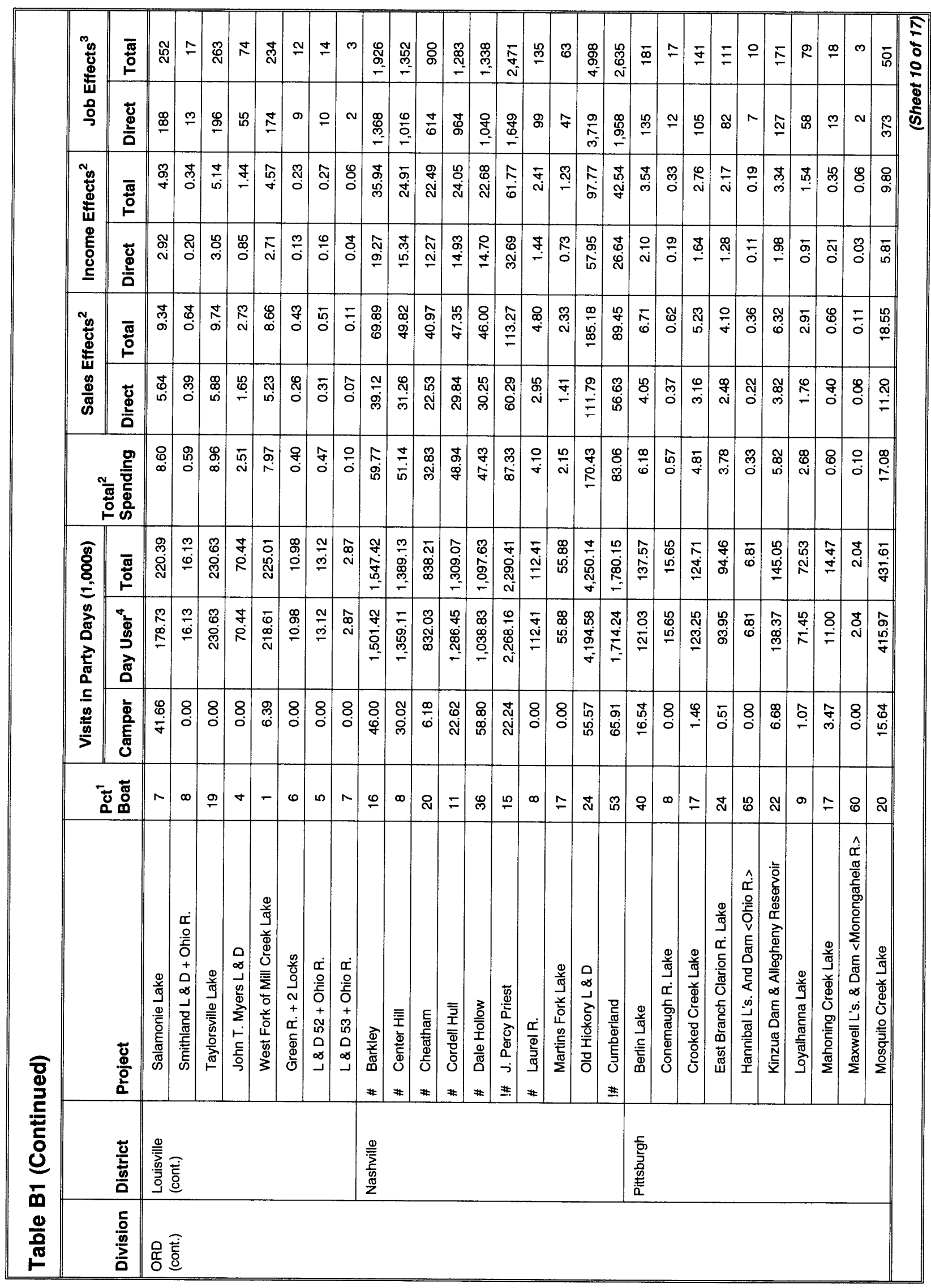




\begin{tabular}{|c|c|c|c|c|c|c|c|c|c|c|c|c|c|c|c|c|c|c|c|c|c|c|c|c|c|c|}
\hline $\begin{array}{l}0 \\
0 \\
0 \\
0\end{array}$ & 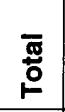 & $f$ & & $=$ & $\begin{array}{l}0 \\
0\end{array}$ & & $\stackrel{\mathscr{0}}{\stackrel{\circ}{\prime}}$ & $\stackrel{\infty}{\sim}$ & $\stackrel{8}{8}$ & 今 & d & & & $\underline{\infty}$ & & & & & + & $\nabla$ & & $\sim$ & & & & \\
\hline 욱 & 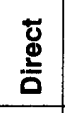 & $\simeq$ & $\circ$ & $r$ & : & $\stackrel{8}{8}$ & 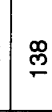 & $\bar{s}$ & I & $\stackrel{8}{\circ}$ & 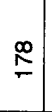 & $\uparrow$ & $\wedge a$ & \pm & $\sigma$ & $\sigma$ & & $v \infty$ & $\infty$ & $\infty$ & D & - & & & 10 & \\
\hline 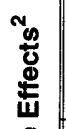 & 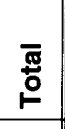 & $\begin{array}{l}0 \\
0 \\
0\end{array}$ & 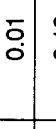 & $\stackrel{8}{\circ}$ & : & 8 & $\underset{\infty}{8}$ & 㕝 & $\stackrel{\mathscr{\&}}{-}$ & ֻัن & 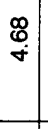 & $\stackrel{\infty}{\circ}$ & $\frac{\infty}{\infty}$ & $\stackrel{\mathscr{D}}{0}$ & $\stackrel{\circ}{\circ}$ & $:$ & ồ & & $\begin{array}{l}0 \\
\vdots \\
o\end{array}$ & âे & o & 年: & $\begin{array}{lll}0 & \vdots \\
\end{array}$ & & Б。 & $\overline{0}_{0}^{\circ}$ \\
\hline $\begin{array}{l}\overline{0} \\
\underline{\underline{g}}\end{array}$ & 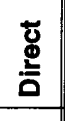 & $\stackrel{9}{0}$ & ¿্: & $\bar{c}=$ & 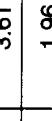 & & $\stackrel{n}{\mathrm{~N}}$ & 恕 & $\stackrel{\circ}{=}$ & 然 & 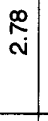 & $\stackrel{0}{\circ}$ & $\begin{array}{l}0 \\
0 \\
0\end{array}$ & गَ & 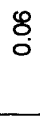 & $\begin{array}{l}8 \\
0 \\
0\end{array}$ & 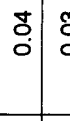 & 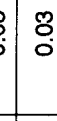 & 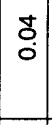 & $\begin{array}{l}\text { t. } \\
\end{array}$ & $s_{S}^{s}$ & مِّ & $\bar{c}$ & $\bar{\partial}$ & $\stackrel{8}{0}$ & : \\
\hline 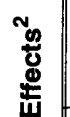 & $\begin{array}{l}\overline{\mathbf{\Xi}} \\
\stackrel{0}{\circ} \\
\end{array}$ & ¿. & : & $\begin{array}{l}\mathscr{c}_{0}^{\circ} \\
\end{array}$ & $\vec{p}$ & $\frac{N}{\infty}$ & \begin{tabular}{|c}
$\hat{\alpha}$ \\
$\hat{\omega}$
\end{tabular} & $\underset{\sim}{\stackrel{0}{0}}$ & $\underset{\infty}{\pi}$ & $\stackrel{\mathscr{L}}{\dot{\sim}}$ & $\begin{array}{c}\hat{\infty} \\
\infty \\
\infty\end{array}$ & 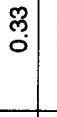 & 胥 & $\stackrel{\infty}{\circ}$ & $\frac{9}{0}$ & $\frac{\infty}{0}$ & $\because \frac{\pi}{0}$ & $\frac{0}{0}$ & \begin{tabular}{|l|} 
\pm \\
0 \\
0
\end{tabular} & $\frac{m}{0}$ & \begin{tabular}{|c|c|} 
\\
\end{tabular} & $\mid \begin{array}{l}8 \\
0 \\
0\end{array}$ & \begin{tabular}{l|l} 
\\
0
\end{tabular} & 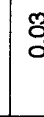 & $\bar{\partial}$ & \\
\hline$\frac{\mathscr{\varpi}}{\mathscr{\varpi}}$ & 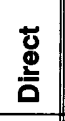 & $\hat{⿳}$ & $\bar{o}$ & $\bar{c}$ & مِ & 8 & $\stackrel{\frac{n}{+}}{+}$ & 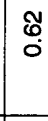 & ָָ & $\stackrel{8}{i}$ & {$\left[\begin{array}{c}\mathscr{0} \\
\vdots \\
\omega\end{array}\right.$} & : & 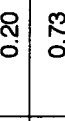 & f & $\stackrel{N}{0}$ & $\overline{0}$ & \begin{tabular}{l|l} 
\\
\hdashline \\
0
\end{tabular} & & \begin{tabular}{l}
0 \\
\hdashline \\
0 \\
0
\end{tabular} & $\begin{array}{l}\text { : } \\
\stackrel{0}{0}\end{array}$ & 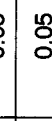 & 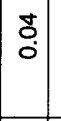 & \begin{tabular}{l|l} 
\\
\end{tabular} & o̊̀ & $\bar{\delta}$ & \\
\hline $\begin{array}{ll}\frac{1}{2} \\
\frac{1}{2}\end{array}$ & 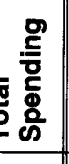 & $\begin{array}{l}: \\
: \\
0\end{array}$ & ؛̊: & 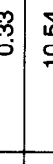 & $\underbrace{\infty}_{i}$ & $\stackrel{g}{9}$ & 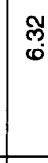 & 离 & $\underset{\varpi}{~}$ & 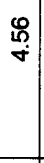 & $\stackrel{\uparrow}{-\infty}$ & 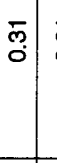 & $=$ & $\stackrel{\square}{\circ}$ & $\frac{\infty}{0}$ & $\stackrel{1}{0}$ & $\bar{c}$ & & $\frac{0}{0}$ & $\div$ & : & $\left|\begin{array}{l}0 \\
0 \\
0\end{array}\right|$ & $\begin{array}{l:l}0 \\
\end{array}$ & $\stackrel{\tilde{O}}{0}$ & চ্ं & : \\
\hline & 嵒 & 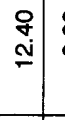 & 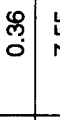 & 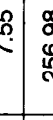 & 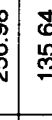 & 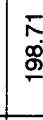 & $\begin{array}{l}\tilde{0} \\
0 \\
0\end{array}$ & 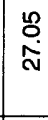 & 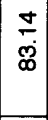 & 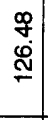 & 辛 & 㤩 & {$\left[\begin{array}{c}5 \\
6 \\
0\end{array}\right.$} & 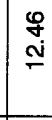 & $\stackrel{0}{0}$ & 总 & 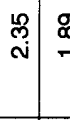 & 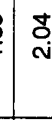 & 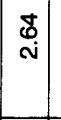 & 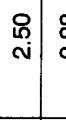 & 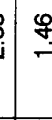 & $\Xi$ & \begin{tabular}{l|l}
$T$ \\
0
\end{tabular} & 点 & $\stackrel{\infty}{\circ}$ & $\frac{0}{5}$ \\
\hline 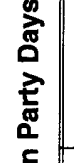 & 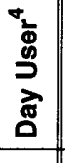 & 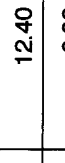 & $\begin{array}{l}0 \\
\vdots \\
0\end{array}$ & 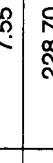 & & 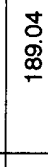 & 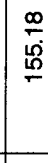 & $\stackrel{\leftrightarrow}{\stackrel{\circ}{*}}$ & 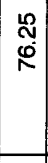 & $\begin{array}{l}\stackrel{8}{\circ} \\
\stackrel{\phi}{=}\end{array}$ & 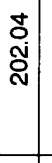 & 离 & 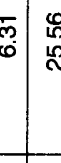 & 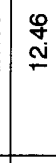 & 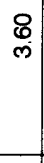 & 总 & 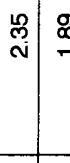 & 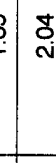 & \begin{tabular}{|l|}
\multirow{d}{*}{} \\
\end{tabular} & 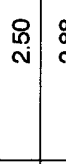 & \begin{tabular}{|l|l} 
\\
\end{tabular} & $\doteqdot$ & & 总 & $\stackrel{\infty}{\circ}$ & \\
\hline$\frac{90}{0}$ & 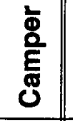 & $\begin{array}{l}8 \\
0\end{array}$ & \&. & \begin{tabular}{l|l}
8 \\
0 \\
0
\end{tabular} & 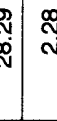 & $\overbrace{\infty}^{\circ}$ & $\underset{\sigma}{\sigma}$ & 8 & 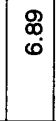 & 䗆 & ఫ. & $\begin{array}{l}8 \\
0\end{array}$ & \begin{tabular}{l|l}
8 & 8 \\
0
\end{tabular} & 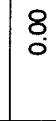 & & $\begin{array}{l}8 \\
0\end{array}$ & 8 & & $\begin{array}{l}0 \\
0\end{array}$ & 8 & $\vec{b}$ & $\stackrel{8}{0}$ & \begin{tabular}{l|l}
8 & 8 \\
0 & 8
\end{tabular} & & 8 & \\
\hline
\end{tabular}

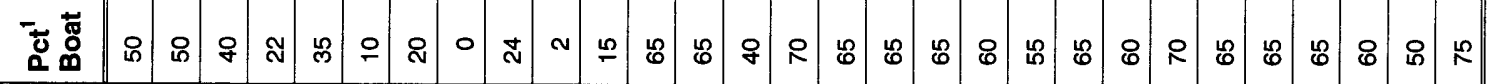

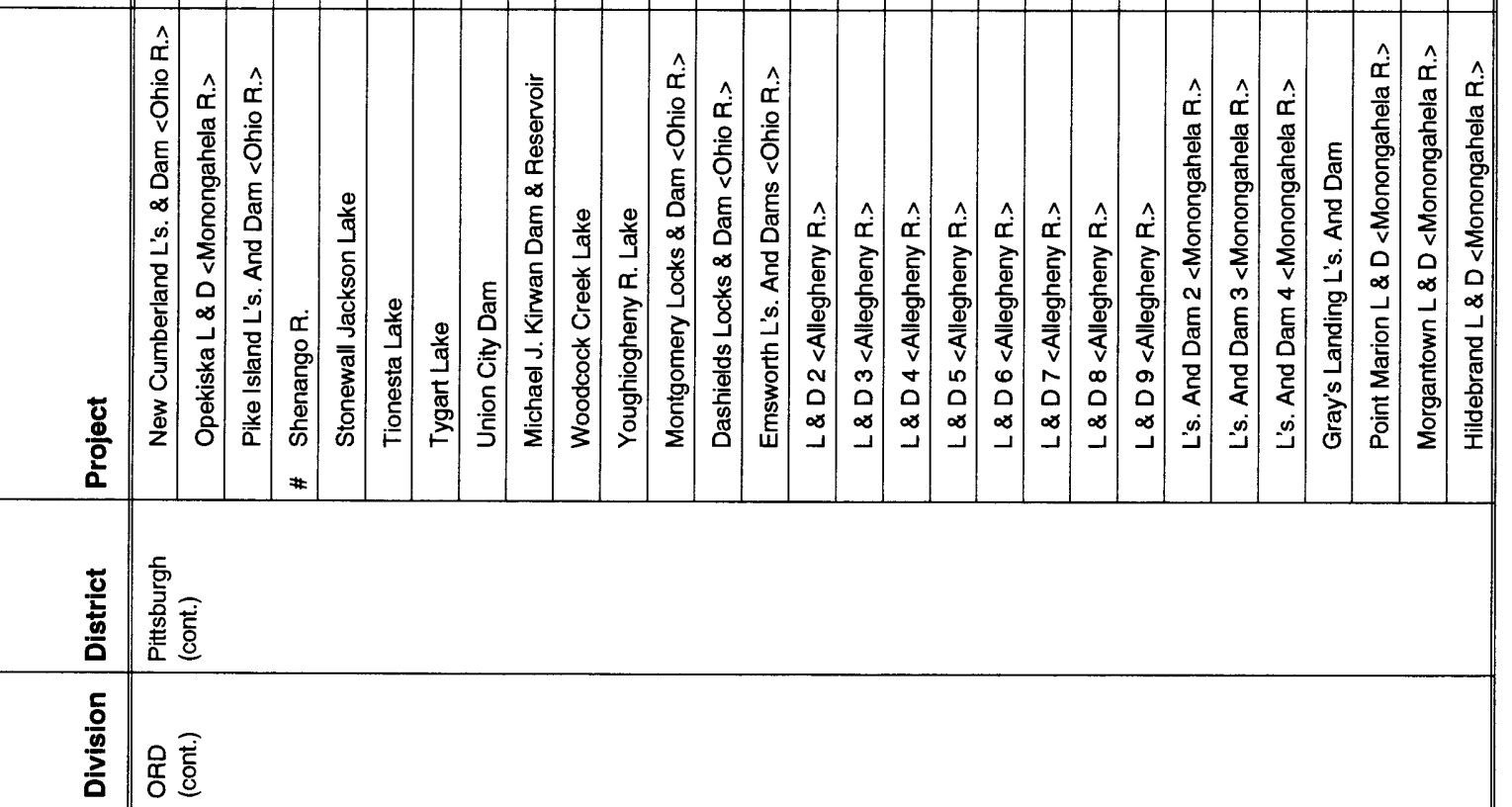




\begin{tabular}{|c|c|c|c|c|c|c|c|c|c|c|c|c|c|c|c|c|c|c|c|c|c|c|c|c|c|c|c|}
\hline & 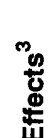 & 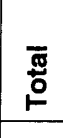 & 8 & $\begin{array}{l}0 \\
\stackrel{0}{s} \\
\text { iv }\end{array}$ & $\bar{N}$ & 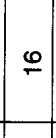 & \begin{tabular}{|l|}
$\infty$ \\
$\frac{0}{N}$ \\
\end{tabular} & $\begin{array}{l}\mathbf{\Phi} \\
\mathbb{\infty}\end{array}$ & $\mid$\begin{tabular}{|c|c}
0 \\
0 \\
s. \\
\end{tabular} & 芯 & ஜ & : & $\stackrel{2}{2}$ & $\bar{n}$ & \& & 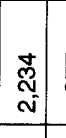 & $\begin{array}{c}\hat{N} \\
-\infty\end{array}$ & $\begin{array}{l}8 \\
\stackrel{8}{\circ} \\
\end{array}$ & 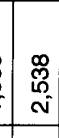 & \begin{tabular}{|c|}
$\begin{array}{c}5 \\
0 \\
i\end{array}$ \\
\end{tabular} & 空 & $\hat{0}$ & & \begin{tabular}{ll}
$\mathscr{\Xi}$ \\
\hdashline
\end{tabular} & 58 & $\begin{array}{lll} \\
\vdots \\
⿱ 亠 乂\end{array}$ & 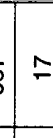 \\
\hline & 웅 & 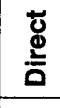 & $\hat{\sigma}$ & 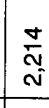 & $\mathscr{0}$ & $\simeq$ & $\begin{array}{r}0 \\
0 \\
0 \\
- \\
\end{array}$ & 总 & 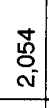 & 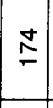 & $\pi$ & $\frac{\infty}{i n}$ & 芦 & 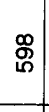 & 跑 & 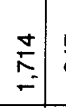 & 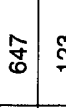 & $\stackrel{\overbrace{}}{\approx}$ & $\begin{array}{l}\bar{\delta} \\
- \\
-\end{array}$ & \begin{tabular}{|l|} 
\\
0 \\
6 \\
-0 \\
\end{tabular} & 胥 & $\infty$ & $\frac{9}{6}$ & $\begin{array}{c}\stackrel{ \pm}{N} \\
\end{array}$ & $\begin{array}{ll} \\
\end{array}$ & \begin{tabular}{l|l}
$b$ \\
$b$ \\
$b$
\end{tabular} & $\simeq$ \\
\hline & 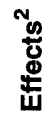 & $\begin{array}{l}\overline{\mathrm{g}} \\
\text { 。 } \\
\end{array}$ & $\stackrel{f}{=}$ & $\underset{\substack{\bar{N} \\
\hdashline}}{\bar{n}}$ & ণั & $\bar{m}$ & 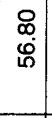 & $\begin{array}{l}\bar{M} \\
\stackrel{n}{\sim}\end{array}$ & $\begin{array}{l}\hat{\hat{p}} \\
\stackrel{i}{i}\end{array}$ & 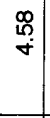 & $\stackrel{\mathscr{\infty}}{-}$ & $\stackrel{\stackrel{m}{=}}{=}$ & $\begin{array}{l}m \\
\dot{j} \\
\dot{\sigma}\end{array}$ & 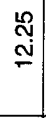 & $\begin{array}{l}8 \\
0 \\
\end{array}$ & $\begin{array}{l}R \\
\hat{m} \\
\hat{m}\end{array}$ & \begin{tabular}{l|l}
$\stackrel{\mathscr{S}}{+}$ \\
\end{tabular} & 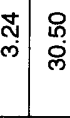 & \begin{tabular}{|c|c|} 
\\
$g$ \\
$g$
\end{tabular} & $\mid \begin{array}{l}0 \\
\dot{d} \\
\underline{0}\end{array}$ & $\begin{array}{c}\infty \\
\infty \\
\sigma\end{array}$ & 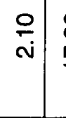 & : & 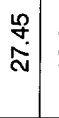 & \begin{tabular}{l|l}
$\stackrel{L}{0}$ \\
$\stackrel{2}{0}$
\end{tabular} & $\frac{5}{7}$ & $\stackrel{\text { ల్ }}{\circ}$ \\
\hline & $\begin{array}{l}\text { Ĕ } \\
\text { ¿ } \\
\text { E }\end{array}$ & 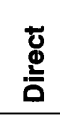 & $\stackrel{\leftrightarrow}{\circ}$ & 总 & \begin{tabular}{|l|l} 
\\
$\stackrel{L}{0}$
\end{tabular} & $\frac{\infty}{0}$ & $\begin{array}{l}\widetilde{\Xi} \\
\dot{\emptyset} \\
\dot{\infty}\end{array}$ & 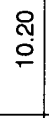 & $\begin{array}{l}\stackrel{0}{\mathscr{g}} \\
\dot{q}\end{array}$ & 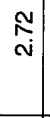 & $\stackrel{\circ}{=}$ & 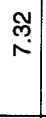 & $\begin{array}{c}\infty \\
\infty \\
\infty \\
\infty\end{array}$ & $\underset{\infty}{\bar{\infty}}$ & $\mid \begin{array}{c}\text { 岕 } \\
\stackrel{\omega}{\circ}\end{array}$ & $\begin{array}{l}\overline{0} \\
\dot{N}\end{array}$ & 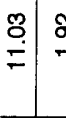 & \begin{tabular}{c|c}
$\stackrel{\infty}{\sim}$ \\
$\stackrel{\infty}{\infty}$
\end{tabular} & 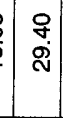 & $\mid \begin{array}{l}0 \\
\stackrel{8}{0} \\
\dot{0}\end{array}$ & $\left|\begin{array}{c}\infty \\
\infty \\
\omega\end{array}\right|$ & $\stackrel{\leftrightarrow}{\stackrel{0}{\varphi}}$ & $\stackrel{N}{\stackrel{N}{=}}$ & $\begin{array}{l}\stackrel{ \pm}{0} \\
\stackrel{0}{0}\end{array}$ & \begin{tabular}{l|l}
$\stackrel{2}{\hat{O}}$ & $\bar{\gamma}$ \\
0 & $\vec{\sigma}$
\end{tabular} & $\begin{array}{lll}5 \\
\end{array}$ & $\frac{9}{0}$ \\
\hline & 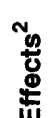 & $\begin{array}{l}\bar{\Xi} \\
\text { 。 }\end{array}$ & 总 & & $\mid \begin{array}{l}R \\
0 \\
0\end{array}$ & $\mid \begin{array}{l}\infty \\
\stackrel{0}{0} \\
0\end{array}$ & \begin{tabular}{c}
\multirow{2}{0}{} \\
$\stackrel{\circ}{\circ}$
\end{tabular} & $\mid$\begin{tabular}{c|}
$\overline{0}$ \\
$\stackrel{j}{m}$
\end{tabular} & $\begin{array}{l}0 \\
0 \\
0 \\
0 \\
0 \\
\end{array}$ & $\begin{array}{l}: \\
\infty \\
\infty\end{array}$ & 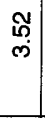 & $\begin{array}{l}\hat{\infty} \\
\stackrel{\sim}{\mathrm{N}}\end{array}$ & $\underset{\overline{\hat{N}}}{\overline{\mathrm{N}}}$ & 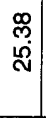 & 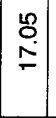 & $\begin{array}{l}\infty \\
\stackrel{\rho}{\sim}\end{array}$ & 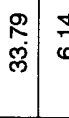 & 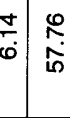 & \begin{tabular}{l}
0 \\
\hdashline \\
8
\end{tabular} & 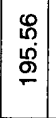 & $\begin{array}{c}\stackrel{0}{\infty} \\
\infty \\
\infty\end{array}$ & $\begin{array}{l}\infty \\
\stackrel{\infty}{\infty} \\
\end{array}$ & $\frac{9}{\frac{\pi}{m}}$ & $\begin{array}{l}\mathfrak{N} \\
\dot{5}\end{array}$ & & & $\overline{0}$ \\
\hline & ळू & 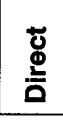 & ָั & $\begin{array}{l}0 \\
0 \\
8 \\
8\end{array}$ & $\begin{array}{l}q \\
\\
0\end{array}$ & $\mid$\begin{tabular}{l}
$\mathscr{0}$ \\
\hdashline \\
0
\end{tabular} & 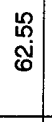 & 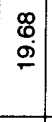 & 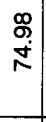 & $\underset{\omega}{\mathbb{S}}$ & $\frac{m}{i}$ & 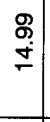 & 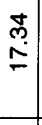 & 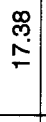 & $\mid \begin{array}{l}0 \\
\stackrel{\rho}{0} \\
\stackrel{0}{0}\end{array}$ & 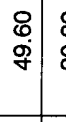 & \begin{tabular}{c|c}
$\infty$ & \\
$\dot{\sim}$ & \\
\end{tabular} & 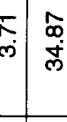 & $\begin{array}{l}\mathbf{D} \\
\mathbf{D} \\
0 \\
0\end{array}$ & $\mid \begin{array}{c}\stackrel{N}{N} \\
\stackrel{1}{=}\end{array}$ & $\stackrel{\stackrel{\overbrace{}}{N}}{=}$ & $\stackrel{g}{\sim}$ & $\overline{\bar{N}}$ & 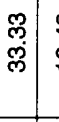 & \begin{tabular}{l|l}
$\stackrel{g}{n}$ \\
$\stackrel{5}{\circ}$
\end{tabular} & & 官 \\
\hline & & 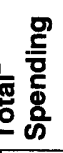 & 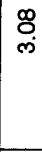 & fo & $\mid \begin{array}{l}0 \\
0 \\
0\end{array}$ & 芯 & $\begin{array}{l}\bar{\delta} \\
\mathscr{\delta}\end{array}$ & $\begin{array}{l}\bar{\delta} \\
\dot{0} \\
\end{array}$ & $\begin{array}{l}\stackrel{0}{2} \\
\stackrel{N}{=}\end{array}$ & $\stackrel{8}{\stackrel{8}{\wedge}}$ & 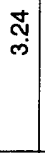 & $\stackrel{\infty}{\dot{d}}$ & $\frac{\mathscr{n}}{\stackrel{\mathrm{N}}{\mathrm{s}}}$ & 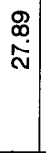 & 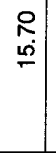 & $\begin{array}{c}\stackrel{\infty}{\infty} \\
\stackrel{\infty}{\infty}\end{array}$ & 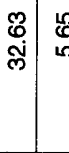 & : & $\mid \begin{array}{c}\infty \\
\infty \\
\infty \\
\infty\end{array}$ & 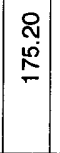 & $\stackrel{N}{\stackrel{N}{ \pm}}$ & $\begin{array}{ll}\& \\
\oplus\end{array}$ & $\begin{array}{l}\stackrel{2}{1} \\
\text { ले }\end{array}$ & 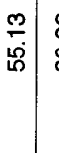 & 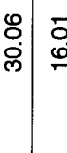 & & $\begin{array}{l}0 \\
0 \\
0 \\
0\end{array}$ \\
\hline & $\begin{array}{l}\overline{8} \\
\overline{8} \\
=\end{array}$ & ङٓ & $\underset{\substack{f \\
\infty \\
\infty}}{R}$ & 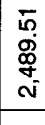 & $\begin{array}{l}8 \\
\stackrel{8}{2} \\
\text { | }\end{array}$ & $\left|\begin{array}{c}0 \\
q \\
\stackrel{1}{r}\end{array}\right|$ & $\begin{array}{l}\frac{\infty}{-} \\
\stackrel{\infty}{-\infty} \\
\stackrel{\sim}{\sim}\end{array}$ & 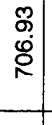 & 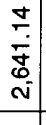 & $\begin{array}{l}q \\
\stackrel{9}{\infty} \\
\infty \\
\infty\end{array}$ & $\begin{array}{l}\mathbb{N} \\
\stackrel{0}{i} \\
\end{array}$ & \begin{tabular}{l}
$\mathbb{0}$ \\
0 \\
0 \\
8 \\
\hdashline
\end{tabular} & $\begin{array}{l}0 \\
0 \\
0 \\
0 \\
i\end{array}$ & $\begin{array}{l}\text { 尺े } \\
\text { oे } \\
0\end{array}$ & 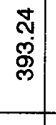 & \begin{tabular}{c|c}
7 & \\
$\omega$ & \\
$\sigma$ & \\
- & \\
- &
\end{tabular} & 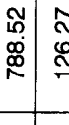 & 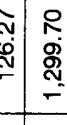 & 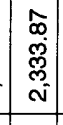 & \begin{tabular}{|l|}
0 \\
0 \\
0 \\
$\vdots$ \\
\end{tabular} & 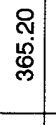 & $\begin{array}{l}\infty \\
0 \\
0 \\
0 \\
0\end{array}$ & 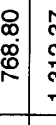 & 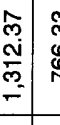 & & \begin{tabular}{l|l}
0 \\
$\vdots$ \\
$\vdots$ \\
\hdashline \\
0
\end{tabular} & 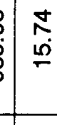 \\
\hline & 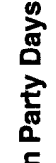 & 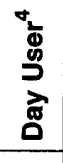 & 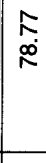 & 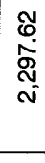 & $\begin{array}{l}\text { o } \\
\text { ¿ें }\end{array}$ & 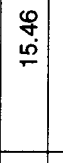 & 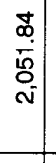 & 备 & 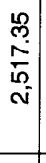 & 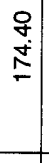 & $\frac{\infty}{\Gamma}$ & 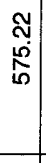 & $\begin{array}{l}\infty \\
0 \\
0 \\
0 \\
0 \\
0\end{array}$ & $\begin{array}{l}\stackrel{8}{\circ} \\
\hat{\circ} \\
\end{array}$ & 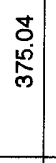 & 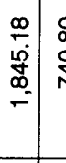 & 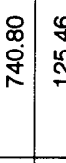 & 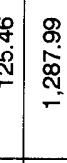 & 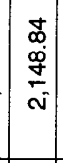 & $\mid \begin{array}{c}0 \\
\stackrel{8}{0} \\
\hat{\tilde{y}} \\
\dot{\sim} \\
\dot{\gamma}\end{array}$ & 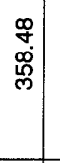 & $\begin{array}{l}\stackrel{\infty}{0} \\
\stackrel{0}{+} \\
\stackrel{0}{0}\end{array}$ & 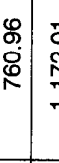 & \begin{tabular}{l|l}
$\bar{c}$ & \\
& \\
&
\end{tabular} & 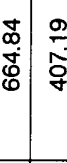 & S & $\begin{array}{l}\stackrel{+}{\hat{0}} \\
\stackrel{\rho}{n}\end{array}$ \\
\hline & 要 & 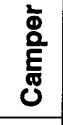 & 8 & $\begin{array}{l}\stackrel{8}{\otimes} \\
\stackrel{-}{\Phi}\end{array}$ & $\begin{array}{l}8 \\
0 \\
0\end{array}$ & \begin{tabular}{l|}
8 \\
0 \\
0
\end{tabular} & 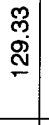 & $\begin{array}{l}R \\
0 \\
0 \\
0\end{array}$ & 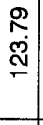 & $\begin{array}{c}\stackrel{8}{\mathscr{\infty}} \\
\infty\end{array}$ & $\underset{8}{+}$ & 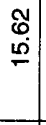 & 足 & $\stackrel{\stackrel{p}{=}}{=}$ & 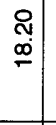 & 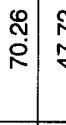 & 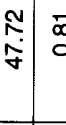 & 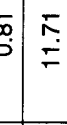 & \begin{tabular}{|c|}
$\stackrel{8}{0}$ \\
$\dot{\rho}$ \\
$\stackrel{\rho}{0}$
\end{tabular} & 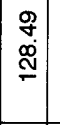 & 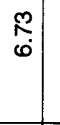 & $\begin{array}{l}8 \\
0\end{array}$ & 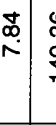 & 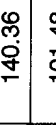 & 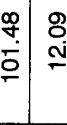 & & 8 \\
\hline & & $\begin{array}{l}0.00 \\
\end{array}$ & న & $\approx$ & 10 & 0 & ల్ల & ㅇ & $\stackrel{8}{\circ}$ & 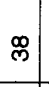 & ले & $\hat{\mathbf{N}}$ & \& & 吕 & $\bar{N}$ & $\begin{array}{lll}8 & :\end{array}$ & ล & \begin{tabular}{l}
$\mathbf{q}$ \\
\hdashline
\end{tabular} & 으 & ल & 台 & - & $\stackrel{\infty}{\infty}:$ & $\widehat{\mathrm{N}} \mathrm{\tau}$ & $\stackrel{ \pm}{ } \pm$ & $\stackrel{\infty}{\infty}$ & $\infty$ \\
\hline $\begin{array}{l}\overline{\mathbf{g}} \\
\mathrm{g}\end{array}$ & & $\begin{array}{l}\frac{\pi}{0} \\
\frac{6}{0} \\
\frac{0}{2}\end{array}$ & 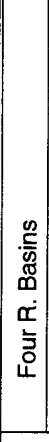 & 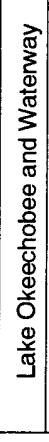 & 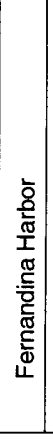 & 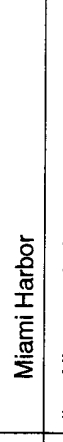 & 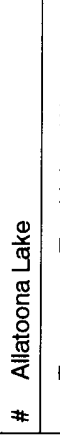 & 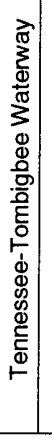 & 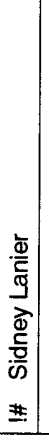 & 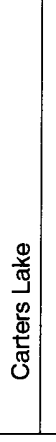 & 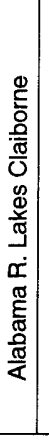 & 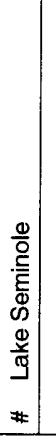 & 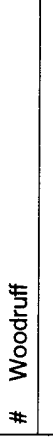 & $\begin{array}{l}\bar{z} \\
\overline{\bar{\Phi}} \\
\overline{5} \\
0 \\
\pm \\
\end{array}$ & 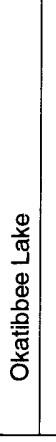 & 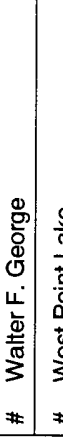 & 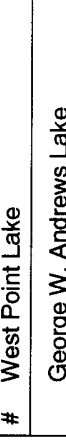 & 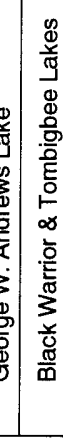 & 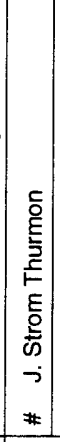 & 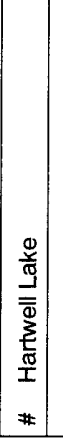 & 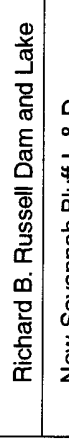 & 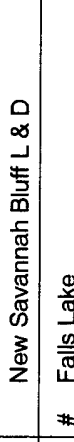 & 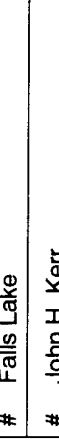 & 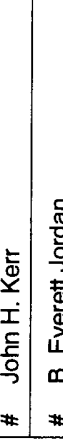 & 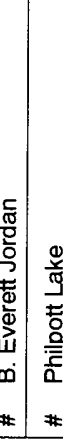 & 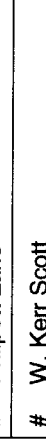 & 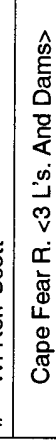 \\
\hline 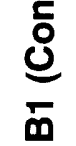 & & $\begin{array}{l}\frac{\overline{\mathrm{O}}}{\mathrm{z}} \\
\frac{\mathrm{m}}{\Delta}\end{array}$ & 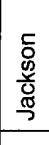 & & & & $\begin{array}{l}\frac{0}{\overline{\bar{O}}} \\
\frac{0}{2}\end{array}$ & & & & & & & & & & & & 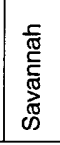 & & & ह & . & & & & \\
\hline$\frac{0}{\frac{0}{0}}$ & & $\frac{\bar{\delta}}{\frac{0}{2}}$ & & & & & & & & & & & & & & & & & & & & & & & & & \\
\hline
\end{tabular}




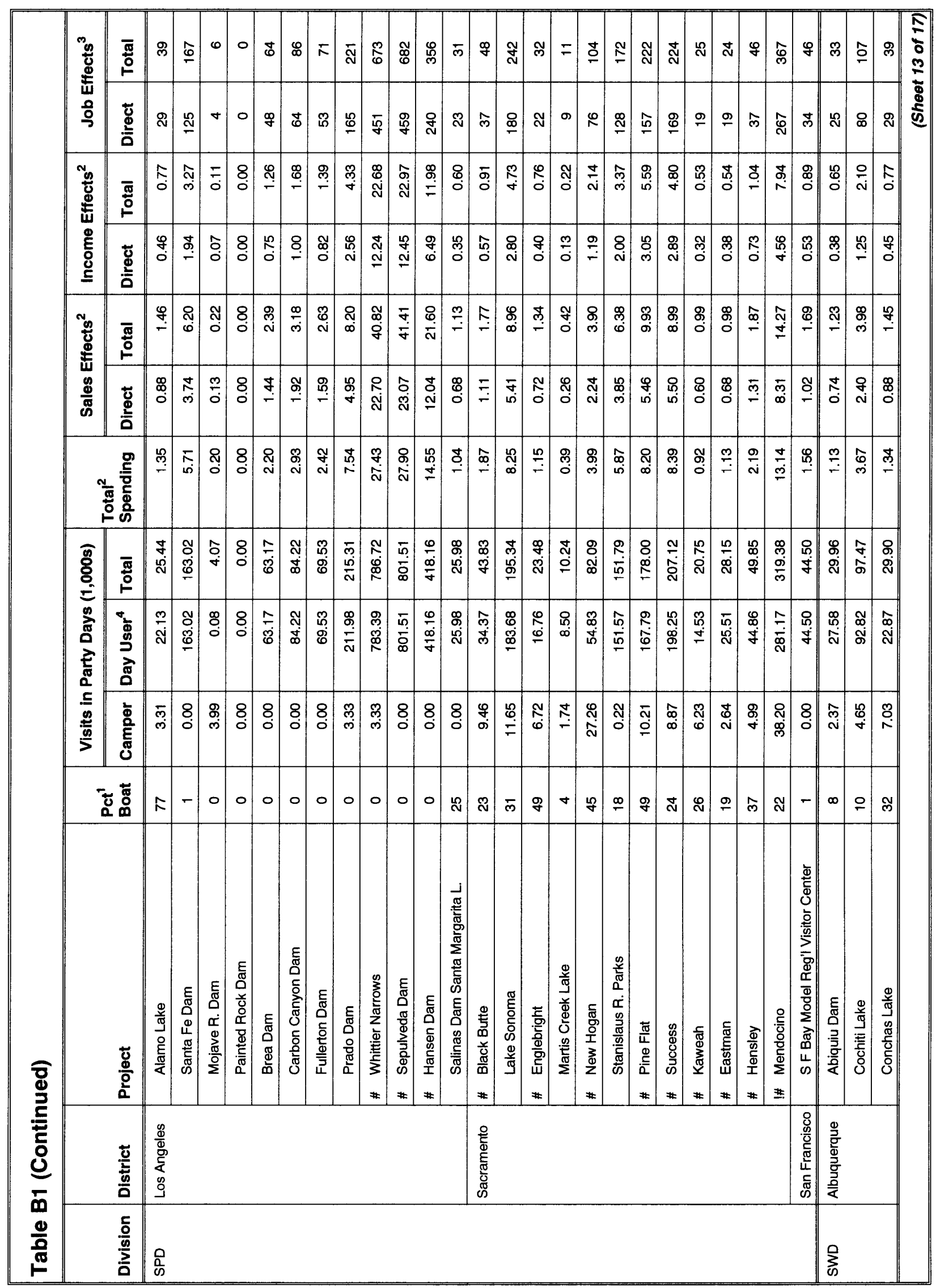




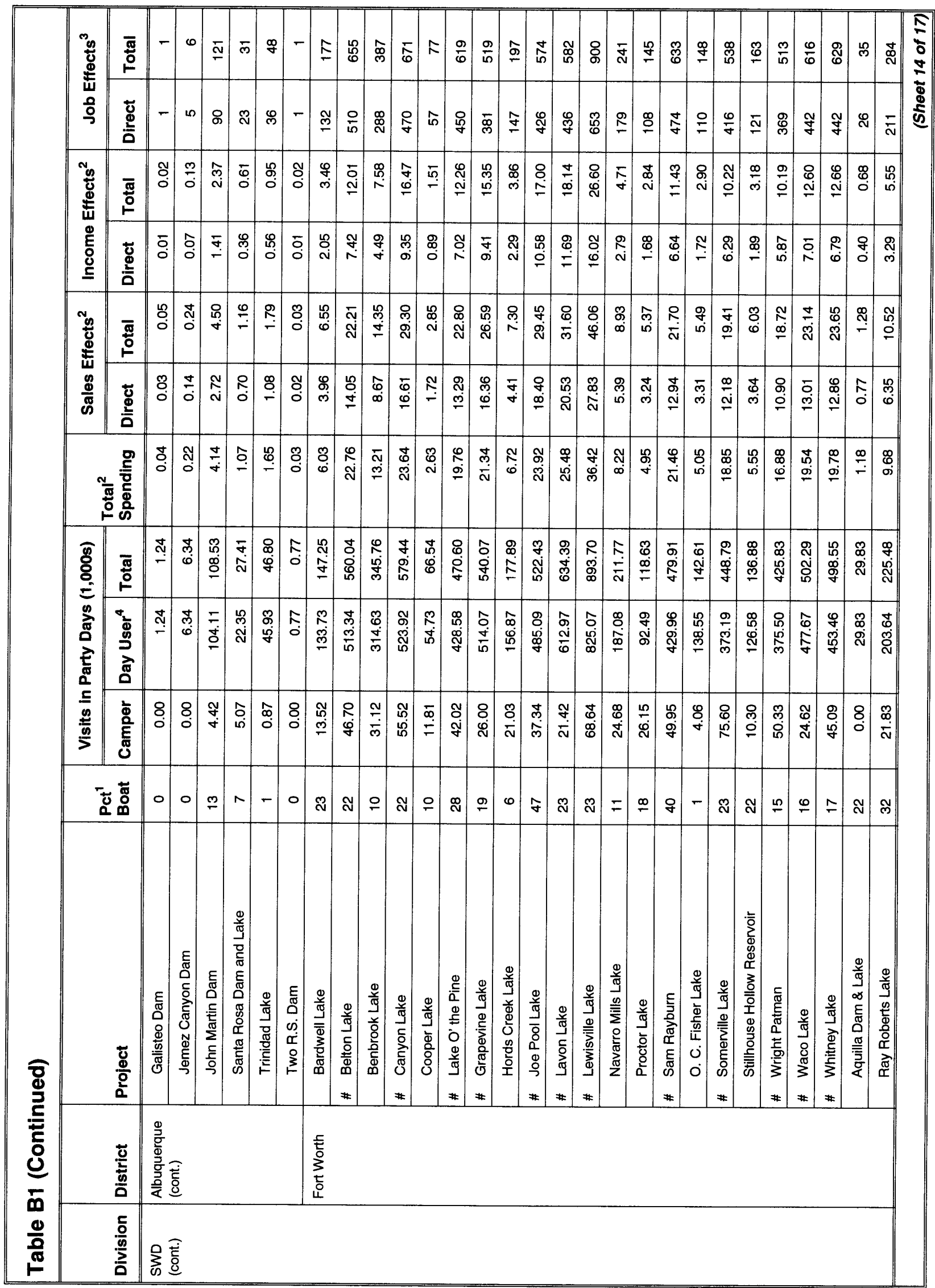




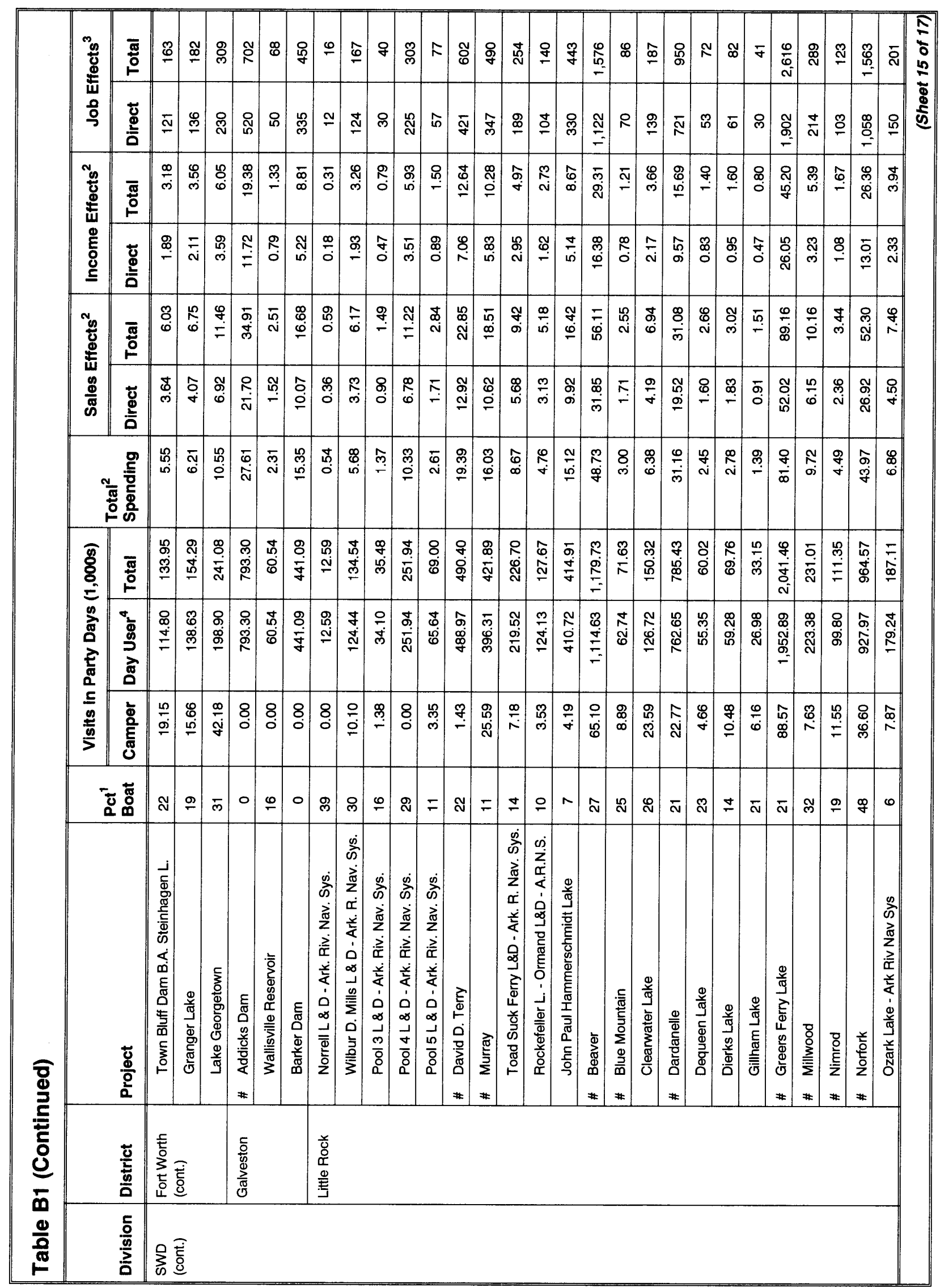




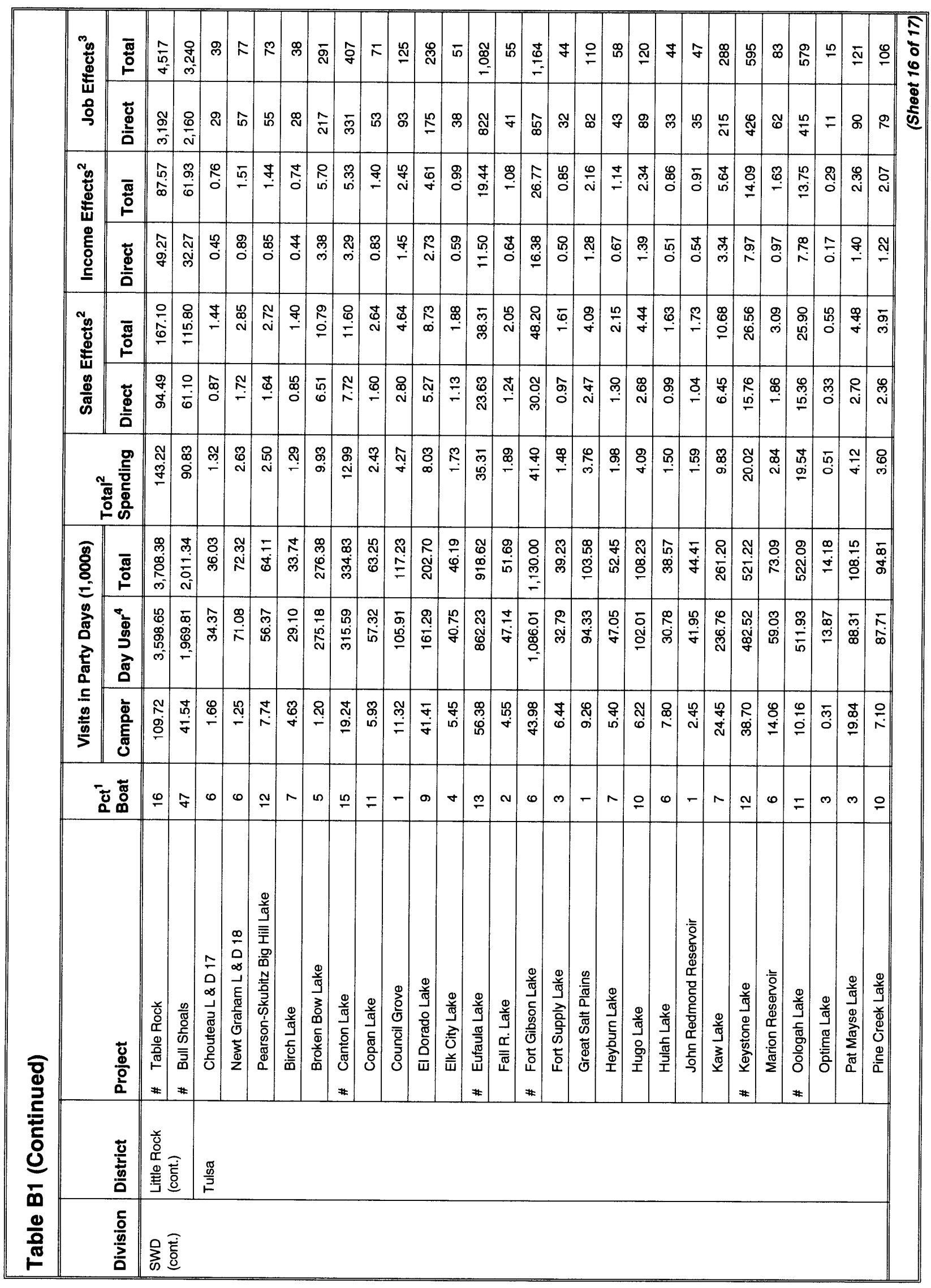




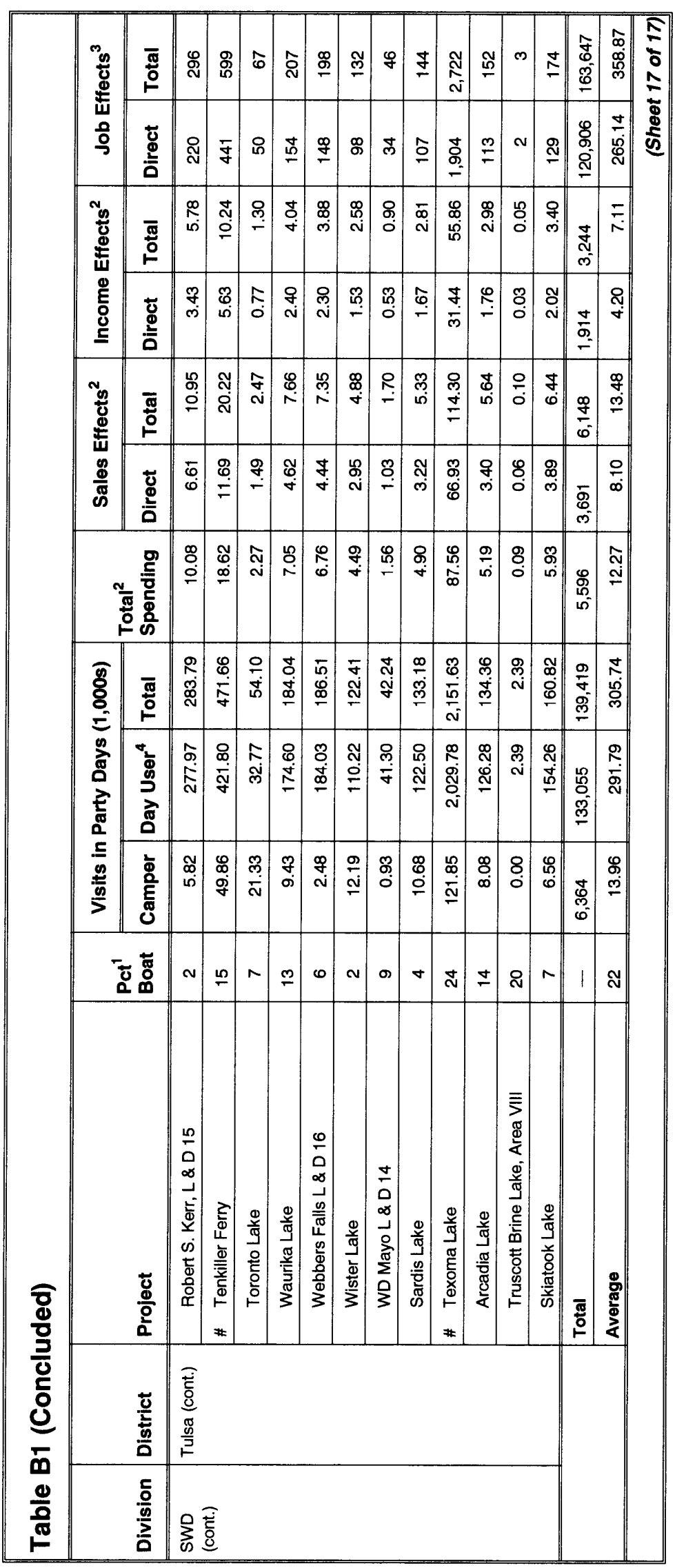




\section{Appendix C Economic Multipliers for Regions Surrounding 108 Corps of Engineers Projects}




\begin{tabular}{|c|c|c|c|c|c|c|c|c|c|c|c|}
\hline \multirow[b]{2}{*}{ Division } & \multirow[b]{2}{*}{ District } & \multirow[b]{2}{*}{ Project } & \multirow{2}{*}{$\begin{array}{l}\text { Capture } \\
\text { Rate }^{2}, \%\end{array}$} & \multicolumn{2}{|c|}{ Sales } & \multicolumn{3}{|c|}{ Income ${ }^{3}$} & \multicolumn{3}{|c|}{ Jobs $^{4}$} \\
\hline & & & & Type I & Type III & Direct & Type I & Type III & Direct & Type I & Type III \\
\hline \multirow[t]{10}{*}{ LMVD } & \multirow[t]{5}{*}{ St. Louis } & $\begin{array}{l}\text { Mark Twain } \\
\text { Lake } \\
\end{array}$ & 62 & 1.21 & 1.74 & 0.50 & 0.60 & 0.88 & 38.00 & 41.01 & 51.52 \\
\hline & & Carlyle Lake & 63 & 1.13 & 1.51 & 0.46 & 0.52 & 0.72 & 36.51 & 38.35 & 45.94 \\
\hline & & Rend Lake & 67 & 1.14 & 1.49 & 0.46 & 0.54 & 0.72 & 32.31 & 34.31 & 41.18 \\
\hline & & Shelbyville & 63 & 1.16 & 1.51 & 0.49 & 0.57 & 0.76 & 33.24 & 35.32 & 41.94 \\
\hline & & Wappapello & 63 & 1.17 & 1.67 & 0.50 & 0.58 & 0.84 & 37.76 & 40.43 & 50.65 \\
\hline & \multirow[t]{5}{*}{ Vicksburg } & Arkabutla Lake & 64 & 1.09 & 1.50 & 0.54 & 0.58 & 0.79 & 31.56 & 32.87 & 40.98 \\
\hline & & Ouachita & 63 & 1.17 & 1.80 & 0.53 & 0.62 & 0.96 & 36.94 & 39.90 & 52.79 \\
\hline & & Degray Lake & 63 & 1.16 & 1.60 & 0.49 & 0.57 & 0.80 & 37.84 & 40.44 & 49.99 \\
\hline & & Grenada Lake & 62 & 1.12 & 1.44 & 0.47 & 0.52 & 0.69 & 36.64 & 38.26 & 44.94 \\
\hline & & Sardis Lake & 63 & 1.12 & 1.49 & 0.47 & 0.52 & 0.72 & 37.75 & 39.35 & 46.87 \\
\hline \multirow[t]{11}{*}{ MRD } & \multirow[t]{6}{*}{ Kansas City } & Harry S. Truman & 63 & 1.21 & 1.73 & 0.47 & 0.57 & 0.84 & 38.59 & 41.71 & 51.76 \\
\hline & & Milford & 63 & 1.23 & 1.69 & 0.44 & 0.55 & 0.80 & 40.19 & 43.97 & 53.35 \\
\hline & & $\begin{array}{l}\text { Pomme de } \\
\text { Terre } \\
\end{array}$ & 60 & 1.17 & 1.77 & 0.43 & 0.52 & 0.83 & 46.89 & 49.66 & 62.01 \\
\hline & & Rathbun Lake & 61 & 1.19 & 1.74 & 0.50 & 0.59 & 0.89 & 38.10 & 41.02 & 51.93 \\
\hline & & Smithville Lake & 67 & 1.22 & 1.95 & 0.54 & 0.67 & 1.07 & 32.91 & 36.33 & 48.79 \\
\hline & & Stockton Lake & 70 & 1.25 & 1.88 & 0.54 & 0.66 & 1.01 & 32.98 & 36.80 & 48.32 \\
\hline & \multirow[t]{5}{*}{ Omaha } & Sharpe & 62 & 1.19 & 1.77 & 0.50 & 0.59 & 0.92 & 39.06 & 42.24 & 53.90 \\
\hline & & Chatfield Lake & 71 & 1.22 & 1.74 & 0.54 & 0.67 & 0.97 & 25.78 & 29.02 & 37.36 \\
\hline & & Cherry Creek & 71 & 1.22 & 1.74 & 0.54 & 0.67 & 0.97 & 25.78 & 29.02 & 37.36 \\
\hline & & Lewis and Clark & 63 & 1.23 & 1.37 & 0.49 & 0.59 & 0.66 & 40.41 & 44.14 & 48.62 \\
\hline & & Oahe & 78 & 1.22 & 1.87 & 0.47 & 0.58 & 0.93 & 32.77 & 36.02 & 48.63 \\
\hline \multirow[t]{2}{*}{ NAD } & Baltimore & Raystown & 63 & 1.15 & 1.65 & 0.54 & 0.61 & 0.89 & 37.49 & 39.91 & 48.94 \\
\hline & Philadelphia & $\begin{array}{l}\text { Blue Marsh } \\
\text { Lake }\end{array}$ & 65 & 1.18 & 1.69 & 0.57 & 0.66 & 0.94 & 31.99 & 34.41 & 42.52 \\
\hline NCD & Rock Island & Saylorville Lake & 67 & 1.28 & 1.99 & 0.55 & 0.71 & 1.11 & 33.22 & 37.40 & 49.75 \\
\hline \multirow[t]{4}{*}{ NPD } & \multirow[t]{4}{*}{ Portland } & Lake Celilo & 62 & 1.15 & 1.57 & 0.57 & 0.65 & 0.88 & 36.56 & 38.85 & 47.24 \\
\hline & & Lake Umatilla & 56 & 1.11 & 1.37 & 0.46 & 0.51 & 0.66 & 38.99 & 40.72 & 46.19 \\
\hline & & Bonneville & 71 & 1.24 & 1.72 & 0.59 & 0.72 & 1.00 & 29.95 & 33.32 & 41.21 \\
\hline & & $\begin{array}{l}\text { Willamette } \\
\text { Lakes }^{5}\end{array}$ & 64 & 1.14 & 1.73 & 0.58 & 0.66 & 0.99 & 36.19 & 37.95 & 49.12 \\
\hline
\end{tabular}

Notes: LMVD = Lower Mississippi Valley; MRD = Missouri River; NAD = North Atlantic; NCD = North Central; NPD = North Pacific; ORD = Ohio River; SAD = South Atlantic $; \mathrm{SPD}=$ South Pacific; SWD = Southwestern.

1 Region defined as all counties within 48-km (30-mile) radius of the project. For the purposes of this report, actual economic impact models have been built for 108 of the total 456 projects.

${ }^{2}$ Capture rate is the percentage of visitor spending captured as direct sales within the region.

3 Income per dollar of direct sales. Income includes employee compensation, proprietor, and other property income.

${ }^{4}$ Jobs per million dollars in direct sales.

${ }^{5}$ Willamette Lakes include Fern Ridge Lake, Cottage Grove Lake, and Fall Creek Lake. These three lakes were treated as a single project in the

12-lake survey (Propst et al. 1992) for sampling purposes. (References cited in this appendix are listed in the References at the end of the main text.) 


\section{Table C1 (Continued)}

\begin{tabular}{|c|c|c|c|c|c|c|c|c|c|c|c|}
\hline \multirow[b]{2}{*}{ Division } & \multirow[b]{2}{*}{ District } & \multirow[b]{2}{*}{ Project } & \multirow{2}{*}{$\begin{array}{l}\text { Capture } \\
\text { Rate }^{2}, \%\end{array}$} & \multicolumn{2}{|c|}{ Sales } & \multicolumn{3}{|c|}{ Income $^{3}$} & \multicolumn{3}{|c|}{ Jobs $^{4}$} \\
\hline & & & & Type I & Type III & Direct & Type I & Type III & Direct & Type I & Type III \\
\hline \multirow{3}{*}{$\begin{array}{l}\text { NPD } \\
\text { (cont.) }\end{array}$} & \multirow[t]{3}{*}{ Walla Walla } & Dworshak & 57 & 1.10 & 1.37 & 0.53 & 0.58 & 0.74 & 40.10 & 42.25 & 48.85 \\
\hline & & Lower Granite & 62 & 1.17 & 1.71 & 0.49 & 0.58 & 0.89 & 40.88 & 43.78 & 55.35 \\
\hline & & McNary & 61 & 1.17 & 1.60 & 0.51 & 0.60 & 0.84 & 35.28 & 37.99 & 46.35 \\
\hline \multirow[t]{20}{*}{ ORD } & \multirow[t]{5}{*}{ Huntington } & $\begin{array}{l}\text { Alum Creek } \\
\text { Lake }\end{array}$ & 66 & 1.22 & 1.66 & 0.59 & 0.70 & 0.95 & 29.99 & 33.05 & 39.87 \\
\hline & & Bluestone Lake & 63 & 1.17 & 1.81 & 0.51 & 0.60 & 0.94 & 34.67 & 37.15 & 49.20 \\
\hline & & $\begin{array}{l}\text { Deer Creek } \\
\text { Lake }\end{array}$ & 66 & 1.21 & 1.62 & 0.59 & 0.71 & 0.93 & 29.66 & 32.68 & 39.00 \\
\hline & & Summersville & 60 & 1.11 & 1.59 & 0.50 & 0.56 & 0.83 & 37.37 & 39.28 & 50.03 \\
\hline & & $\begin{array}{l}\text { Senecaville } \\
\text { Lake }\end{array}$ & 66 & 1.14 & 1.59 & 0.53 & 0.59 & 0.83 & 34.63 & 36.68 & 45.19 \\
\hline & \multirow[t]{6}{*}{ Louisville } & Barren R. Lake & 64 & 1.19 & 1.68 & 0.51 & 0.60 & 0.86 & 36.48 & 39.92 & 49.64 \\
\hline & & $\begin{array}{l}\text { William H. } \\
\text { Harsha }\end{array}$ & 68 & 1.24 & 1.78 & 0.59 & 0.72 & 1.03 & 28.93 & 32.30 & 40.95 \\
\hline & & Cecil M. Harden & 64 & 1.15 & 1.59 & 0.52 & 0.60 & 0.84 & 37.14 & 39.57 & 48.21 \\
\hline & & Monroe Lake & 62 & 1.17 & 1.57 & 0.52 & 0.60 & 0.82 & 35.61 & 38.21 & 46.07 \\
\hline & & Nolin R. Lake & 63 & 1.19 & 1.60 & 0.50 & 0.60 & 0.81 & 37.35 & 40.63 & 48.86 \\
\hline & & Rough R. Lake & 62 & 1.17 & 1.56 & 0.48 & 0.57 & 0.77 & 39.29 & 41.95 & 49.73 \\
\hline & \multirow[t]{8}{*}{ Nashville } & Barkley & 65 & 1.20 & 1.79 & 0.49 & 0.60 & 0.92 & 34.97 & 37.98 & 49.22 \\
\hline & & Center Hill & 61 & 1.16 & 1.59 & 0.49 & 0.57 & 0.80 & 32.50 & 34.98 & 43.23 \\
\hline & & Cheatham & 69 & 1.25 & 1.82 & 0.54 & 0.68 & 1.00 & 27.24 & 30.84 & 39.94 \\
\hline & & Cordell Hull & 61 & 1.15 & 1.59 & 0.50 & 0.58 & 0.81 & 32.31 & 34.87 & 43.01 \\
\hline & & Dale Hollow & 64 & 1.15 & 1.52 & 0.49 & 0.56 & 0.75 & 34.38 & 36.82 & 44.25 \\
\hline & & J. Percy Priest & 69 & 1.25 & 1.88 & 0.54 & 0.68 & 1.02 & 27.35 & 30.93 & 40.99 \\
\hline & & Laurel R. & 72 & 1.16 & 1.63 & 0.49 & 0.57 & 0.82 & 33.65 & 36.25 & 45.90 \\
\hline & & Cumberland & 68 & 1.15 & 1.58 & 0.47 & 0.53 & 0.75 & 34.58 & 37.22 & 46.54 \\
\hline & Pittsburgh & Shenango R. & 67 & 1.19 & 1.85 & 0.52 & 0.61 & 0.98 & 36.15 & 38.95 & 50.65 \\
\hline \multirow[t]{9}{*}{ SAD } & \multirow[t]{7}{*}{ Mobile } & Allatoona Lake & 67 & 1.21 & 1.56 & 0.59 & 0.70 & 0.91 & 26.16 & 28.77 & 34.18 \\
\hline & & Sidney Lanier & 67 & 1.20 & 1.74 & 0.58 & 0.69 & 0.99 & 27.39 & 30.09 & 38.85 \\
\hline & & Lake Seminole & 62 & 1.15 & 1.53 & 0.49 & 0.56 & 0.76 & 34.21 & 36.46 & 43.97 \\
\hline & & Woodruff & 66 & 1.20 & 1.56 & 0.51 & 0.62 & 0.81 & 30.79 & 33.89 & 40.68 \\
\hline & & Dannelly & 62 & 1.15 & 1.46 & 0.47 & 0.54 & 0.70 & 34.42 & 36.64 & 43.21 \\
\hline & & $\begin{array}{l}\text { Walter F. } \\
\text { George }\end{array}$ & 59 & 1.16 & 1.53 & 0.48 & 0.56 & 0.76 & 34.56 & 36.99 & 45.04 \\
\hline & & West Point Lake & 64 & 1.17 & 1.62 & 0.53 & 0.61 & 0.86 & 30.96 & 33.60 & 41.99 \\
\hline & \multirow[t]{2}{*}{ Savannah } & $\begin{array}{l}\text { J. Strom } \\
\text { Thurmon } \\
\end{array}$ & 64 & 1.19 & 1.64 & 0.52 & 0.62 & 0.86 & 33.41 & 36.36 & 44.62 \\
\hline & & Hartwell Lake & 67 & 1.20 & 1.67 & 0.52 & 0.63 & 0.89 & 31.36 & 34.34 & 42.75 \\
\hline
\end{tabular}




\section{Table C1 (Continued)}

\begin{tabular}{|c|c|c|c|c|c|c|c|c|c|c|c|}
\hline \multirow[b]{2}{*}{ Division } & \multirow[b]{2}{*}{ District } & \multirow[b]{2}{*}{ Project } & \multirow{2}{*}{$\begin{array}{l}\text { Capture } \\
\text { Rate }^{2}, \%\end{array}$} & \multicolumn{2}{|c|}{ Sales } & \multicolumn{3}{|c|}{ Income $^{3}$} & \multicolumn{3}{|c|}{ Jobs $^{4}$} \\
\hline & & & & Type I & Type III & Direct & Type I & Type III & Direct & Type I & Type III \\
\hline \multirow{5}{*}{$\begin{array}{l}\text { SAD } \\
\text { (cont.) }\end{array}$} & \multirow[t]{5}{*}{ Wilmington } & Falls Lake & 64 & 1.13 & 1.48 & 0.56 & 0.62 & 0.82 & 30.69 & 32.12 & 38.08 \\
\hline & & John H. Kerr & 60 & 1.15 & 1.64 & 0.49 & 0.56 & 0.82 & 38.24 & 40.45 & 49.95 \\
\hline & & $\begin{array}{l}\text { B. Everett } \\
\text { Jordan }\end{array}$ & 65 & 1.13 & 1.49 & 0.55 & 0.62 & 0.82 & 30.81 & 32.28 & 38.53 \\
\hline & & Philpott Lake & 59 & 1.12 & 1.53 & 0.50 & 0.56 & 0.78 & 37.85 & 40.34 & 48.55 \\
\hline & & W. Kerr Scott & 62 & 1.11 & 1.48 & 0.52 & 0.57 & 0.77 & 35.32 & 36.86 & 44.31 \\
\hline \multirow[t]{12}{*}{ SPD } & \multirow[t]{3}{*}{ Los Angeles } & Whittier Narrows & 83 & 1.25 & 1.80 & 0.54 & 0.68 & 1.00 & 19.87 & 22.73 & 29.66 \\
\hline & & Sepulveda Dam & 83 & 1.25 & 1.79 & 0.54 & 0.68 & 1.00 & 19.91 & 22.74 & 29.55 \\
\hline & & Hansen Dam & 83 & 1.25 & 1.79 & 0.54 & 0.68 & 1.00 & 19.91 & 22.74 & 29.55 \\
\hline & \multirow[t]{9}{*}{ Sacramento } & Black Butte & 59 & 1.14 & 1.60 & 0.51 & 0.58 & 0.82 & 33.62 & 35.66 & 43.59 \\
\hline & & Englebright & 63 & 1.18 & 1.87 & 0.55 & 0.66 & 1.05 & 30.37 & 33.20 & 44.23 \\
\hline & & New Hogan & 56 & 1.15 & 1.74 & 0.53 & 0.61 & 0.95 & 33.87 & 36.20 & 46.35 \\
\hline & & Pine Flat & 67 & 1.24 & 1.82 & 0.56 & 0.69 & 1.02 & 28.68 & 32.06 & 40.74 \\
\hline & & Success & 66 & 1.22 & 1.64 & 0.53 & 0.64 & 0.87 & 30.66 & 33.88 & 40.71 \\
\hline & & Kaweah & 66 & 1.22 & 1.64 & 0.53 & 0.64 & 0.87 & 30.66 & 33.88 & 40.71 \\
\hline & & Eastman & 60 & 1.09 & 1.43 & 0.56 & 0.60 & 0.79 & 27.98 & 29.41 & 35.06 \\
\hline & & Hensley & 60 & 1.09 & 1.43 & 0.56 & 0.60 & 0.79 & 28.02 & 29.42 & 35.02 \\
\hline & & Mendocino & 63 & 1.18 & 1.72 & 0.55 & 0.65 & 0.96 & 32.08 & 34.91 & 44.15 \\
\hline \multirow[t]{17}{*}{ SWD } & \multirow[t]{12}{*}{ Fort Worth } & Belton Lake & 62 & 1.16 & 1.58 & 0.53 & 0.62 & 0.86 & 36.31 & 39.11 & 46.64 \\
\hline & & Canyon Lake & 70 & 1.21 & 1.76 & 0.56 & 0.68 & 0.99 & 28.29 & 31.42 & 40.43 \\
\hline & & Lake O' the Pine & 67 & 1.18 & 1.72 & 0.53 & 0.62 & 0.92 & 33.85 & 36.57 & 46.55 \\
\hline & & Grapevine Lake & 77 & 1.20 & 1.62 & 0.58 & 0.69 & 0.94 & 23.29 & 25.73 & 31.72 \\
\hline & & Joe Pool Lake & 77 & 1.20 & 1.60 & 0.57 & 0.69 & 0.92 & 23.13 & 25.57 & 31.19 \\
\hline & & Lavon Lake & 81 & 1.19 & 1.54 & 0.57 & 0.68 & 0.88 & 21.25 & 23.53 & 28.32 \\
\hline & & Lewisville Lake & 76 & 1.20 & 1.66 & 0.58 & 0.69 & 0.96 & 23.46 & 25.93 & 32.35 \\
\hline & & Sam Rayburn & 60 & 1.17 & 1.68 & 0.51 & 0.60 & 0.88 & 36.67 & 39.29 & 48.92 \\
\hline & & Somerville Lake & 65 & 1.17 & 1.59 & 0.52 & 0.61 & 0.84 & 34.12 & 36.60 & 44.18 \\
\hline & & Wright Patman & 65 & 1.17 & 1.72 & 0.54 & 0.63 & 0.93 & 33.82 & 36.69 & 47.04 \\
\hline & & Waco Lake & 67 & 1.20 & 1.78 & 0.54 & 0.65 & 0.97 & 33.97 & 37.11 & 47.37 \\
\hline & & Whitney Lake & 65 & 1.18 & 1.84 & 0.53 & 0.62 & 0.98 & 34.36 & 37.18 & 48.90 \\
\hline & Galveston & Addicks Dam & 79 & 1.19 & 1.61 & 0.54 & 0.65 & 0.89 & 23.96 & 26.28 & 32.34 \\
\hline & \multirow[t]{4}{*}{ Little Rock } & David D. Terry & 67 & 1.23 & 1.77 & 0.55 & 0.68 & 0.98 & 32.60 & 36.77 & 46.59 \\
\hline & & Murray & 66 & 1.23 & 1.74 & 0.55 & 0.68 & 0.97 & 32.65 & 36.82 & 46.12 \\
\hline & & Beaver & 65 & 1.22 & 1.76 & 0.51 & 0.63 & 0.92 & 35.24 & 39.27 & 49.47 \\
\hline & & Blue Mountain & 57 & 1.15 & 1.49 & 0.45 & 0.52 & 0.71 & 40.94 & 43.20 & 50.39 \\
\hline
\end{tabular}




\begin{tabular}{|c|c|c|c|c|c|c|c|c|c|c|c|}
\hline \multirow[b]{2}{*}{ Division } & \multirow[b]{2}{*}{ District } & \multirow[b]{2}{*}{ Project } & \multirow{2}{*}{$\begin{array}{l}\text { Capture } \\
\text { Rate }^{2}, \%\end{array}$} & \multicolumn{2}{|c|}{ Sales } & \multicolumn{3}{|c|}{ Income $^{3}$} & \multicolumn{3}{|c|}{ Jobs $^{4}$} \\
\hline & & & & Type I & Type III & Direct & Type I & Type III & Direct & Type I & Type III \\
\hline \multirow{14}{*}{$\begin{array}{l}\text { SWD } \\
\text { (cont.) }\end{array}$} & \multirow{7}{*}{$\begin{array}{l}\text { Little Rock } \\
\text { (cont.) }\end{array}$} & Dardanelle & 63 & 1.17 & 1.59 & 0.49 & 0.58 & 0.80 & 36.96 & 39.83 & 48.66 \\
\hline & & $\begin{array}{l}\text { Greers Ferry } \\
\text { Lake }\end{array}$ & 64 & 1.17 & 1.71 & 0.50 & 0.59 & 0.87 & 36.56 & 39.32 & 50.29 \\
\hline & & Millwood & 63 & 1.17 & 1.65 & 0.53 & 0.62 & 0.88 & 34.77 & 37.69 & 46.95 \\
\hline & & Nimrod & 53 & 1.14 & 1.46 & 0.45 & 0.53 & 0.71 & 43.36 & 45.51 & 51.82 \\
\hline & & Norfork & 61 & 1.16 & 1.94 & 0.48 & 0.57 & 0.98 & 39.29 & 42.25 & 58.05 \\
\hline & & Table Rock & 66 & 1.21 & 1.77 & 0.52 & 0.62 & 0.93 & 33.78 & 37.09 & 47.80 \\
\hline & & Bull Shoals & 67 & 1.18 & 1.90 & 0.53 & 0.62 & 1.01 & 35.35 & 38.49 & 53.03 \\
\hline & \multirow[t]{7}{*}{ Tulsa } & Canton Lake & 59 & 1.18 & 1.50 & 0.43 & 0.52 & 0.69 & 42.80 & 46.24 & 52.68 \\
\hline & & Eufaula Lake & 67 & 1.19 & 1.62 & 0.49 & 0.59 & 0.82 & 34.79 & 37.86 & 45.81 \\
\hline & & $\begin{array}{l}\text { Fort Gibson } \\
\text { Lake }\end{array}$ & 73 & 1.13 & 1.61 & 0.55 & 0.62 & 0.89 & 28.54 & 30.55 & 38.79 \\
\hline & & Keystone Lake & 79 & 1.22 & 1.69 & 0.51 & 0.63 & 0.89 & 27.01 & 30.23 & 37.74 \\
\hline & & Oologah Lake & 79 & 1.22 & 1.69 & 0.51 & 0.63 & 0.90 & 27.04 & 30.29 & 37.72 \\
\hline & & Tenkiller Ferry & 63 & 1.22 & 1.73 & 0.48 & 0.60 & 0.88 & 37.69 & 41.46 & 51.29 \\
\hline & & Texoma Lake & 76 & 1.17 & 1.71 & 0.47 & 0.56 & 0.83 & 28.44 & 31.08 & 40.68 \\
\hline \multicolumn{3}{|c|}{ Average } & 66 & 1.18 & 1.66 & 0.52 & 0.61 & 0.87 & 33.27 & 36.00 & 44.71 \\
\hline \multicolumn{12}{|r|}{ 'age 4 of 4) } \\
\hline
\end{tabular}




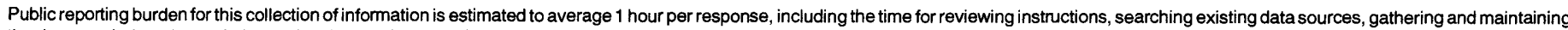

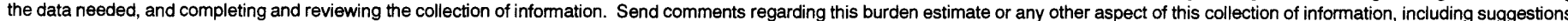

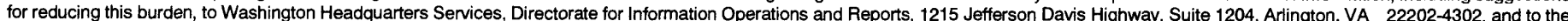
Office of Management and Budget, Paperwork Reduction Project (0704-0188), Washington, DC 20503.
1. AGENCY USE ONLY (Leave blank)
2. REPORT DATE
September 1998

\section{REPORT TYPE AND DATES COVERED} Final report

\section{TITLE AND SUBTITLE}

Estimating the Local Economic Impacts of Recreation at Corps of Engineers

Projects - 1996

\section{AUTHOR(S)}

Dennis B. Propst, Daniel J. Stynes, Wen-Huei Chang, R. Scott Jackson

\section{PERFORMING ORGANIZATION NAME(S) AND ADDRESS(ES)}

Department of Park and Recreation Resources, Michigan State University, East Lansing, MI 48824; U.S. Army Engineer Waterways Experiment Station, 3909 Halls Ferry Road, Vicksburg, MS 39180-6199

\section{SPONSORING/MONITORING AGENCY NAME(S) AND ADDRESS(ES)}

U.S. Army Corps of Engineers

10. SPONSORING/MONITORING Washington, DC 20314-1000

5. FUNDING NUMBERS

8. PERFORMING ORGANIZATION REPORT NUMBER

Technical Report R-98-1

\section{SUPPLEMENTARY NOTES}

Available from National Technical Information Service, 5285 Port Royal Road, Springfield, VA 22161.

12a. DISTRIBUTION/AVAILABILITY STATEMENT

Approved for public release; distribution is unlimited. 12b. DISTRIBUTION CODE

\section{ABSTRACT (Maximum 200 words)}

This report presents separate project-level estimates of the economic effects of visitor spending within local regions surrounding Corps of Engineers (CE) projects. In 1996, visitors to CE projects spent over $\$ 5.5$ billion on trip-related goods and services in local regions (within $48 \mathrm{~km}$ (30 miles)) around CE projects. This spending was associated with over $\$ 3.2$ in income and 163 thousand jobs in these local regions. It is recognized that significant visitor spending and associated economic activity occur outside the regions adjacent to $\mathrm{CE}$ projects. However, it is important to understand local economic effects because they may be particularly sensitive to management actions affecting recreation-use patterns and spending behavior.

14. SUBJECT TERMS Corps of Engineers

Economic impact

Recreation

Visitor spending

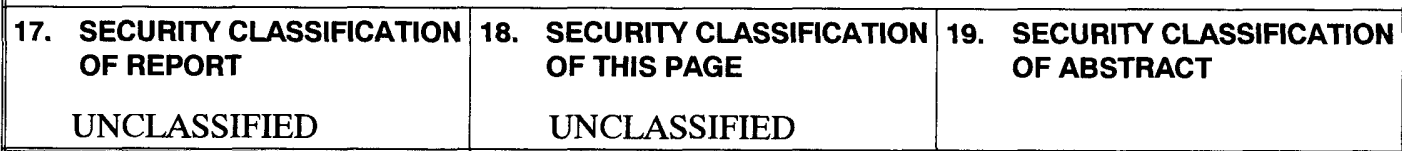
NSN 7540-01-280-5500
15. NUMBER OF PAGES 63

16. PRICE CODE

20. LIMITATION OF ABSTRACT

Standard Form 298 (Rev. 2-89) Prescribed by ANSI Std. Z39-18 298-102 
Destroy this report when no longer needed. Do not return it to the originator. 